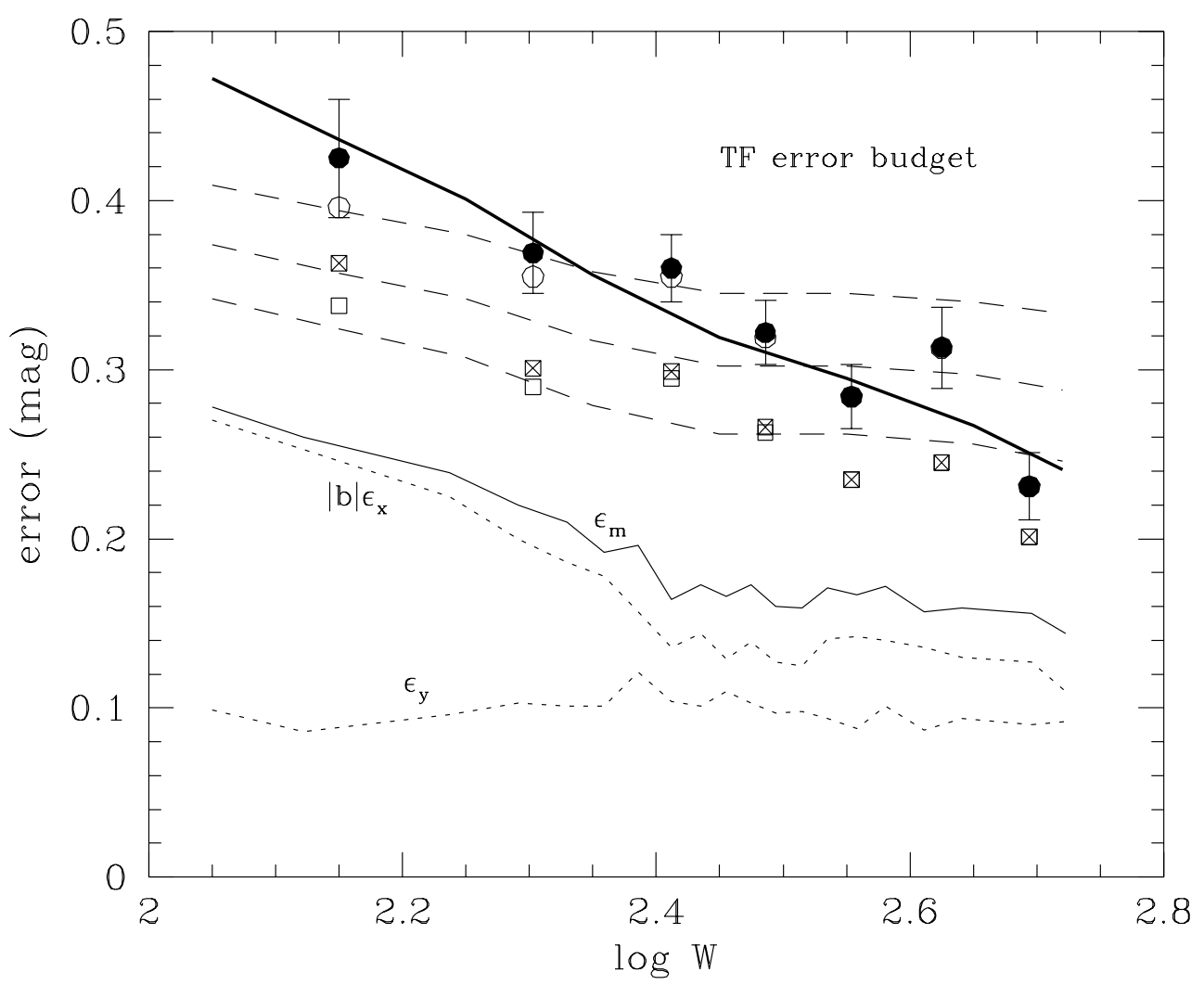

Fig. 1 
TABLE 1

Incompleteness and Size Bias Coeffs.

\begin{tabular}{|c|c|c|c|c|c|c|c|}
\hline $\begin{array}{c}\text { Cluster Name } \\
\text { (1) }\end{array}$ & $\begin{array}{l}y_{F} \quad \dagger \\
(2)\end{array}$ & $\begin{array}{c}\eta \\
(3)\end{array}$ & $\begin{array}{l}y_{F} \\
(4)\end{array}$ & $\begin{array}{c}\eta \\
(5)\end{array}$ & $\begin{array}{c}\beta_{s i z, i n} \\
(6)\end{array}$ & $\begin{array}{c}\beta_{s i z, i n+} \\
\quad(7)\end{array}$ & (8) \\
\hline NGC 383 & -19.55 & 0.80 & -20.50 & 0.63 & -0.002 & -0.005 & $*$ \\
\hline NGC 507 & -19.55 & 0.80 & -20.50 & 0.63 & -0.002 & -0.004 & $*$ \\
\hline A262 & -20.80 & 0.77 & -21.47 & 0.43 & -0.002 & -0.004 & * \\
\hline $\mathrm{A} 400$ & -20.65 & 0.25 & -20.80 & 0.21 & -0.002 & -0.004 & * \\
\hline Eridanus & -17.30 & 0.17 & -18.05 & 0.30 & -0.012 & -0.012 & \\
\hline Fornax & -16.30 & 0.10 & -16.95 & 0.35 & -0.012 & -0.012 & \\
\hline Cancer & -19.20 & 0.07 & -19.35 & 0.22 & -0.003 & -0.010 & * \\
\hline Antlia & -20.05 & 0.57 & -20.90 & 0.42 & -0.005 & -0.010 & \\
\hline Hydra & -20.30 & 0.45 & -20.65 & 0.26 & -0.003 & -0.011 & $*$ \\
\hline NGC 3557 & -20.05 & 0.57 & -20.90 & 0.42 & -0.004 & -0.004 & \\
\hline A1367 & -20.80 & 0.50 & -21.30 & 0.46 & -0.002 & -0.003 & $*$ \\
\hline Ursa Major & -17.45 & 0.22 & -18.60 & 1.17 & -0.012 & -0.012 & \\
\hline Cen 30 & -18.55 & 0.13 & -19.85 & 0.86 & -0.003 & -0.010 & \\
\hline Coma & -20.85 & 0.47 & -21.30 & 0.51 & -0.003 & -0.004 & $*$ \\
\hline ESO 508 & -19.45 & 0.27 & -19.70 & 0.26 & -0.004 & -0.006 & \\
\hline A3574 & -20.80 & 0.73 & -21.30 & 0.51 & -0.002 & -0.008 & $*$ \\
\hline A 2197 & -22.20 & 0.30 & -22.25 & 0.27 & -0.001 & -0.002 & $*$ \\
\hline A2199 & -22.20 & 0.30 & -22.25 & 0.27 & -0.001 & -0.002 & $*$ \\
\hline Pavo 2 & -20.80 & 0.50 & -21.20 & 0.31 & -0.002 & -0.005 & $*$ \\
\hline Pavo & -19.90 & 0.40 & -20.28 & 0.30 & -0.003 & -0.010 & \\
\hline MDL59 & -18.10 & 0.15 & -18.80 & 0.75 & -0.006 & -0.010 & \\
\hline Pegasus & -18.75 & 0.35 & -19.05 & 0.41 & -0.003 & -0.005 & \\
\hline A2634 & -21.65 & 0.45 & -21.85 & 0.32 & -0.002 & -0.002 & $*$ \\
\hline A2666 & -21.55 & 0.75 & -22.00 & 0.60 & -0.002 & & $*$ \\
\hline
\end{tabular}

${ }^{\dagger}$ Cols. 2 and 3: completeness function parameters for $\alpha=-0.50$. $\ddagger$ Cols. 4 and 5: completeness function parameters for $\alpha=-0.85$.

*Used for definition of CMB reference frame. 

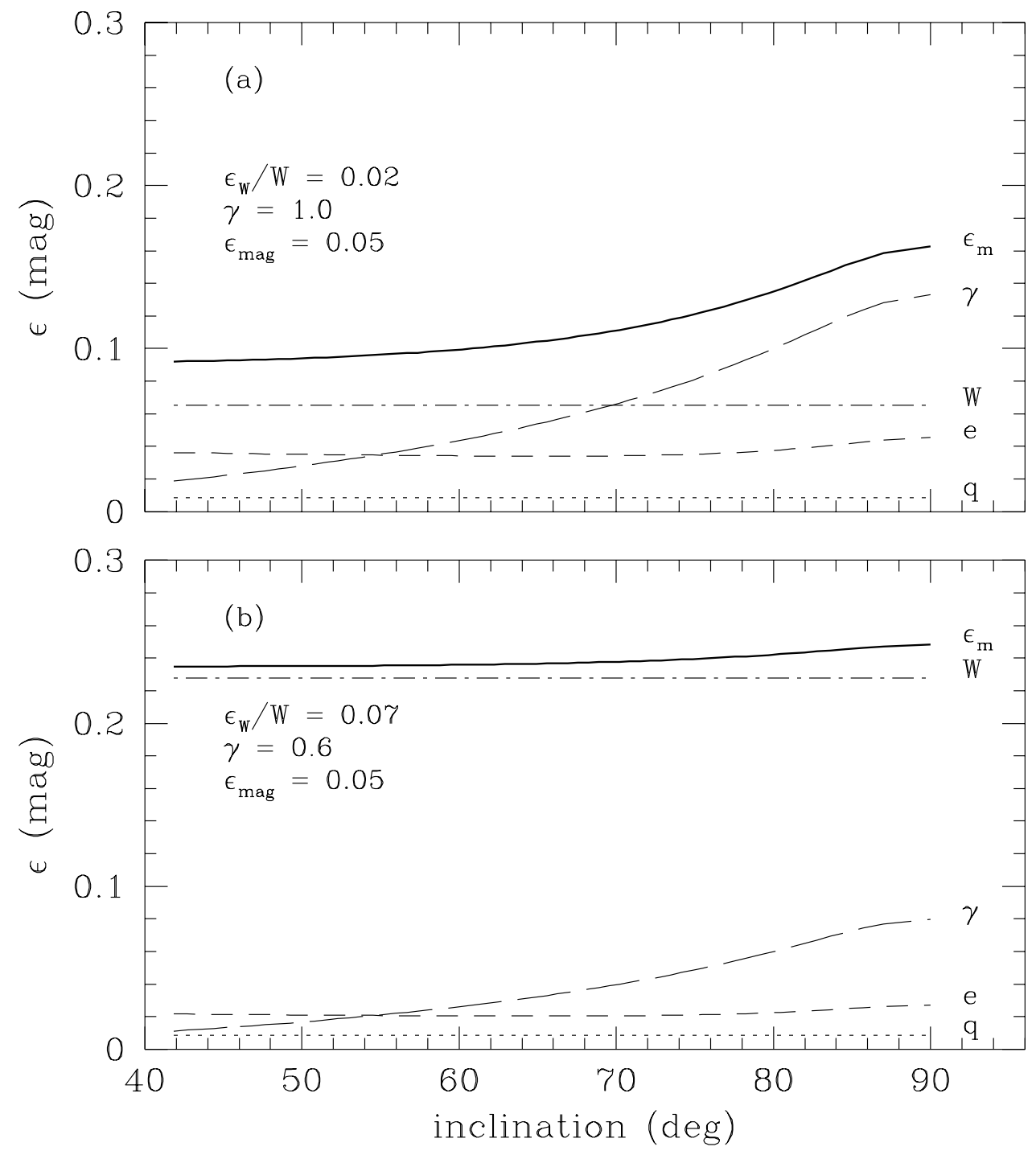

fig. 2 
Version $1.6 \quad 14$ Oct 1996

To appear in Astronomical Journal

\title{
The I-Band Tully-Fisher Relation for Cluster Galaxies: A Template Relation, its Scatter and Bias Corrections
}

\author{
Riccardo Giovanelli, Martha P. Haynes, Terry Herter and Nicole P. Vogt \\ Center for Radiophysics and Space Research and National Astronomy and Ionosphere Center ${ }^{1}$, \\ Cornell University, Ithaca, NY 14853 \\ Luiz N. da Costa \\ European Southern Observatory, Karl-Schwarzschild-Str. 2, D-85748 Garching b. München, \\ Germany and Observatorio Nacional, Rio de Janeiro, Brazil \\ Wolfram Freudling \\ Space Telescope-European Coordinating Facility and European Southern Observatory, \\ Karl-Schwarzschild-Str. 2, D-85748 Garching b. München, Germany \\ John J. Salzer \\ Astronomy Dept., Wesleyan University, Middletown, CT 06459 \\ Gary Wegner \\ Dept. of Physics and Astronomy, Dartmouth College, Hanover, NH 03755
}

\begin{abstract}
Infrared I band photometry and velocity widths for galaxies in 24 clusters, with radial velocities between 1,000 and $10,000 \mathrm{~km} \mathrm{~s}^{-1}$, are used to construct a template Tully-Fisher (TF) relation. The sources of scatter in the TF diagram are analyzed in detail; it is shown that the common practice of referring to a single figure of $\mathrm{TF}$ scatter is incorrect and can lead to erroneous bias corrections. Biases resulting from sample incompleteness, catalog inaccuracies, cluster size and other sources, as well as dependences of TF parameters on morphological type and local environment, are discussed and appropriate corrections are obtained. A template TF relation is constructed by combining the data from the 24 clusters, and kinematic cluster offsets from a putative reference frame which well approximates null velocity with respect to the cosmic microwave background, are obtained.
\end{abstract}

Subject headings: galaxies: distances and redshifts; photometry - cosmology: observations; cosmic microwave background

\footnotetext{
${ }^{1}$ The National Astronomy and Ionosphere Center is operated by Cornell University under a cooperative agreement with the National Science Foundation.
} 


\section{Introduction}

This is the seventh paper of a series based on spectroscopy and I band photometry of spiral galaxies, obtained with the purpose of improving the calibration of the Tully-Fisher (TF) relation (Tully and Fisher 1977) and our understanding of the peculiar velocity field in the local universe. Previous papers in the series investigated the adequacy of internal extinction corrections for spiral disks (Giovanelli et al. 1994 and Giovanelli et al. 1995a, hereafter respectively referred to as Paper I and Paper II); developed techniques for the correction of selection biases in the determination of peculiar velocities of field galaxies (Freudling et al. 1995, hereafter Paper III); tested the evidence for large scale bulk flows (Giovanelli et al. 1996a; hereafter paper IV); presented a reconstruction of the density field (da Costa et al. 1996; hereafter paper V); and provided a data set of galaxy parameters in cluster fields (Giovanelli et al. 1996b, hereafter Paper VI). The analysis described here is based on the data presented in Paper VI, which appears as a companion paper in this issue. Preliminary reports of this work have appeared in Giovanelli et al. (1995b), which are superseded by this paper. The motivation for this study is given in the introduction of Paper VI.

In Paper VI we present data for 782 spiral galaxies in the fields of 24 clusters in both hemispheres, with systemic velocities spread between $c z \sim 10^{3} \mathrm{~km} \mathrm{~s}^{-1}$ and $c z \sim 10^{4} \mathrm{~km} \mathrm{~s}^{-1}$, previously and throughout this paper referred to as the SCI sample. The tabulated parameters were derived from our own observations or were collated from other sources in the public domain. The treatment of the data was carried out in such a way as to produce a homogeneous set of TF parameters, which appear in Table 2 of Paper VI.

Cluster membership was assigned to each galaxy in the sample on the basis of criteria somewhat more stringent than generally adopted in previous TF work. Galaxies associated with each cluster were separated into two classes: (i) an in sample includes galaxies that are very likely to be cluster members, on the basis of their sky and redshift coordinates; (ii) a class of "peripheral" objects includes objects having a redshift quite close to the systemic one of the cluster, but sufficiently removed spatially from the cluster center so that a membership assignment cannot be reliably made. The combination of in objects and peripheral objects is referred to as the in+ sample. The global in sample of the 24 clusters includes 374 galaxies, of which 360 are deemed good candidates for TF work, while the in+ sample includes 584 objects, of which 555 are used for TF work. The remaining 198 galaxies in paper VI are either foreground or background objects, or members of groups/clusters inadequately sampled for the purposes of this study. Sample assignments and justifications for exclusion are given in Table 2 of Paper VI.

Here we obtain TF fits to each of the 24 clusters and combine the data to produce a template TF relation. Such a template relation will be adopted in the analysis of the peculiar velocity field of clusters, as well as that of an all sky sample of field spiral galaxies (the SFI sample), which will be presented elsewhere.

In section 2 we analyze in detail the characteristics of the TF error budget, while in section 3

we discuss morphological type differences and the important issue of bias. In section 4 we describe 
the procedures adopted in obtaining $\mathrm{TF}$ fits to the data, while in section 5 we present the TF relations fit separately to each of the 24 clusters. In section 6 we combine the cluster samples and obtain a global template. In section 7 we investigate the residuals with respect to that template. The results are summarized in section 8. In the next paper of this series (Giovanelli et al 1996c: paper VIII) we analyze the cluster motions with respect to the reference frame defined by the cluster set.

Throughout this paper, we will often refer to the absolute magnitude variable as

$$
y=M+5 \log h
$$

where $h=H_{\circ} /\left(100 \mathrm{~km} \mathrm{~s}^{-1} \mathrm{Mpc}^{-1}\right)$ and $M$ is the I band absolute magnitude of a galaxy. Absolute magnitudes are tabulated in Paper VI on the assumptions that: (a) galaxies in the in samples are at the distance indicated by the systemic velocity of the cluster measured in the Cosmic Microwave Background (CMB) reference frame; this assumption implies that the clusters are at rest with respect to the $\mathrm{CMB}$; (b) peripheral galaxies that complete the in + samples are at the distance indicated by each galaxy's radial velocity. The assumption that individual clusters are at rest will in general be incorrect; magnitude offsets that take into account cluster motions need to be applied in order to obtain correct values of $y$. We give an estimate of such offsets in section 6 .

We shall also define a velocity width variable

$$
x=\log W-2.5
$$

which will equal zero near $W=300 \mathrm{~km} \mathrm{~s}^{-1}$, where $W$ is an estimate of twice the maximum rotational velocity of a galaxy. This convention is dictated by the practice of deriving $W$ from Doppler-broadened single-dish $21 \mathrm{~cm}$ line global profiles of galaxies. The relationship between the measured linewidth and the rotational speed of the galaxy is discussed in paper VI and, in further detail, by Haynes et al. (1997).

\section{Error Budget of the TF Relation}

The process of obtaining a template TF relation by combining several cluster TF diagrams requires an understanding of the characteristics of the error budget. This is necessary in the estimate of the amplitude of the corrections which take into account the effects of known biases affecting the process. We thus review those characteristics before the procedures and corrections applied to obtain a template relation are discussed.

In this section, we shall then run ahead of ourselves, assume that the template TF relation is known, and analyze residuals and measurement errors about that relation. We do so for the bivariate fit (see eqn. 18) to the sample displayed in Figures 21(b) and 21(c), the in+ 
composite which includes all cluster members and peripheral objects as discussed in section 6.3. The magnitude offsets produced by peculiar velocities of individual clusters are removed before residuals are computed. The magnitude residual for the $i$-th galaxy is defined as $r_{i}=y_{i}-\left(a+b x_{i}\right)$ where $(a, b)$ is the "bivariate" TF relation of slope $b=-7.68$ and offset $a=-21.01$.

Obtaining a reliable TF fit requires bias corrections which in turn require a knowledge of the characteristics of the scatter. The empirical determination of the latter requires that a reliable TF relation be known. The circularity of the process forces an iterative approach, which fortunately converges rapidly to a stable solution.

\subsection{The Total Observed Scatter}

In Figure 1 we summarize our estimates of the error budget in the TF diagram. The mean absolute deviation and the standard deviation of the observed residuals, averaged within bins in velocity width, are plotted respectively as unfilled squares and circles. The observed values do however underestimate the scatter: as discussed in section 3.2, sample incompleteness depresses the measured values of the scatter at low velocity widths. A correction for the incompleteness bias is applied to the observed estimates of the scatter, shifting the points up: the bias corrected values of the scatter are represented by filled circles and crossed squares. The locations of bias-corrected and measured scatter amplitudes coincide for larger values of the velocity width. Indicative uncertainties for each bin are plotted as error bars about the corrected standard deviations; similar uncertainties apply to the values of the mean absolute deviations.

Note the decreasing trend in the scatter, with increasing velocity width. A linear fit to the scatter represented by the solid symbols is:

$$
\epsilon_{t o t}=-0.325 x+0.32 \operatorname{mag}
$$

Deviations from the TF relation arise from three main causes:

(i) measurement errors affecting the estimates of raw magnitudes, inclinations and velocity widths;

(ii) uncertainties arising from the corrections applied to measured parameters, such as those for extinction, turbulent motions, etc.;

(iii) "cosmic" scatter, an umbrella term for everything else, including effects as varied as velocity field distortions, deviations from disk planarity, other gravitational and photometric asymmetries, variations in the stellar population make-up, variations in disk-to-bulge ratios, in cluster samples' depth, etc.

Uncertainties arising from sources (i) and (ii) are estimated as described in sections 4, 5 and 6 of paper VI; we refer to them as $\epsilon_{x}$ and $\epsilon_{y}$, where $\epsilon_{x}=0.434 \epsilon_{w} / W$ and $\epsilon_{w}$ is the error on the velocity 
width as given in eqns. (6) and (8) of paper VI. $\epsilon_{x}$ and $\epsilon_{y}$ are listed in Table 2 of paper VI, for each galaxy in the SCI sample.

\subsection{Measurement and Correction Errors}

What is the impact of measurement and correction errors on the TF scatter? Given a generic function of two variables $f(x, y)$, the standard error propagation formula indicates that

$$
\epsilon_{f}^{2}=\epsilon_{x}^{2}\left(\frac{\partial f}{\partial x}\right)^{2}+\epsilon_{y}^{2}\left(\frac{\partial f}{\partial y}\right)^{2}+2 \epsilon_{x y}\left(\frac{\partial f}{\partial x}\right)\left(\frac{\partial f}{\partial y}\right)
$$

In most cases, errors in $x$ and in $y$ are uncorrelated and the covariant term $\epsilon_{x y}$ is assumed to be null. In the case of the TF variables, however, errors in the two coordinates are correlated, mainly via the inclination dependences which correct velocity widths to the edge-on perspective and magnitudes to a face- on aspect by means of an internal extinction term. It is thus more convenient to compute the scatter on the distance modulus inferred from the TF correction by expressing it in terms of all the directly measurable quantities separately, so that error contributions appear expressed in ways that can be easily compared. The TF parameters $(x, y)$ in the relation

$$
y=a+b x
$$

are obtained via the following correction recipes (see paper VI):

$$
\begin{gathered}
x=\log \left[\left(\frac{W_{o b s}-\Delta_{s}}{1+z}-\Delta_{t}\right) / \sin i-2.5\right] \\
y=m_{o b s}-A_{I}-\Delta m_{i}+k_{I}-5 \log \frac{c z-V_{p e c}}{100 h}-25
\end{gathered}
$$

where $m_{o b s}$ and $W_{o b s}$ are the observed magnitude and width; $\Delta_{s}$ is the width correction that accounts for the effects of instrumental broadening, signal-to-noise distortions and data processing smoothing; $\Delta_{t}$ is the correction for turbulent motions; $i$ is the disk inclination; $A_{I}$ is the extinction arising in our Galaxy; $\Delta m_{i}$ is the internal extinction term; $k_{I}$ is the cosmological correction that

accounts for the shift of the spectral energy distribution of the galaxy; $V_{p e c}$ is the galaxy's peculiar velocity, $z$ its redshift and $c$ the speed of light (see paper VI for details on each of the correction terms). The internal extinction term is defined in paper VI as

$$
\Delta m_{i}=-\gamma \log (1-e)
$$

where $e$ is the ellipticity, corrected for seeing, and $\gamma$ is a function of galaxy luminosity (or velocity width). The inclination is derived from the ellipticity via 


$$
\cos ^{2} i=\frac{(1-e)^{2}-q_{\circ}^{2}}{1-q_{\circ}^{2}}
$$

where $q_{\circ}$ is the intrinsic axial ratio of the spiral disk, for which in paper VI we have assumed different values according to galaxy type.

Neglecting the contributions to errors arising from uncertainties on the redshift $z$ (typically less than 1\%), the extinction within the Milky Way (typically less than $0.02 \mathrm{mag}$ ), the $k_{I}$ term (typically less than $0.01 \mathrm{mag}$ ), and assuming a null peculiar velocity field, the error contributions on the various observed and assumed quantities propagate to the distance modulus via:

$$
\epsilon_{m}^{2}=\epsilon_{m a g}^{2}+d_{\gamma}^{2} \epsilon_{\gamma}^{2}+d_{W}^{2} \epsilon_{W}^{2}+d_{q}^{2} \epsilon_{q}^{2}+d_{e}^{2} \sigma_{e}^{2}
$$

where

$$
\begin{gathered}
d_{\gamma}=\log (1-e) \\
d_{W}=0.434 b / W \\
d_{q}=0.434 b q_{\circ} /\left(1-q_{\circ}^{2}\right) \\
d_{e}=\frac{0.434 \gamma}{1-e}+\frac{0.434 b(1-e)}{1-(1-e)^{2}}
\end{gathered}
$$

Above, we have substituted (see section 5, Paper VI):

$$
W=\left[\left(W_{o b s}-\Delta_{s}\right)^{2} /(1+z)^{2}-\Delta_{t}^{2}\right]^{1 / 2}
$$

which is the velocity width corrected for all but the disk inclination, so that

$$
\epsilon_{W}^{2}=\frac{\epsilon_{W, o b s}^{2}+\epsilon_{s}^{2}}{(1+z)^{2}}+\epsilon_{t}^{2}
$$

where $\epsilon_{W, o b s}$ is the total measurement error on the width. For the remaining terms, we assume the uncertainties:

$$
\begin{gathered}
\epsilon_{q}=0.15 q_{\circ}, \\
\epsilon_{\gamma}=0.15 \gamma, \\
\epsilon_{s}=0.25 \Delta_{s}, \\
\epsilon_{t}=0.25 \Delta_{t} .
\end{gathered}
$$


In Figure 2, we illustrate the relative contributions of the various components to the total measurement error $\epsilon_{m}$, in two different cases. Curves in Figure 2 have been calculated using the mean trend indicated by eqn. (3) of paper VI, for the ellipticity; an intrinsic axial ratio for disks of $q_{\circ}=0.13$; a TF slope $b=-7.67$; a measurement error on the magnitude of $\epsilon_{\text {mag }}=0.05 \mathrm{mag}$ and the indicated values of $\gamma$. The curves labelled $\gamma, W, q_{\circ}$ and $e$ correspond to the terms $\left|d_{\gamma} \epsilon_{\gamma}\right|$, $\left|d_{W} \epsilon_{W}\right|,\left|d_{q} \epsilon_{q}\right|$ and $\left|d_{e} \epsilon_{e}\right|$. In panel (a), which might correspond to a relatively bright galaxy ( $\gamma$ is large) with well determined width $\left(\epsilon_{W} / W=0.02\right)$, contributions to $\epsilon_{m}$ due to width, magnitude and ellipticity measurements are roughly equivalent; at high inclinations, the uncertainty on the value of $\gamma$ becomes dominant. On the other hand, panel (b) might correspond to the case of a narrow width, low luminosity object (low value of $\gamma$ ), for which the relative error on the width $\left(\epsilon_{W} / W=0.07\right)$ becomes the dominant source of $\epsilon_{m}$. In the latter case, the total measurement error is also substantially larger than in the first. This simulation helps illustrate important characteristics of the TF error budget, some of which are often misunderstood. First, except in exceptional cases errors in the photometry are generally negligible in comparison to those arising from other sources, such as the measurement and correction of velocity widths and those produced by internal extinction corrections. Second, velocity width measurement and correction errors are frequently, but not always the most important contribution to the total measurement error. Corrections for internal extinction can dominate the total measurement error, e.g. for highly inclined, fast rotators.

\subsection{The Intrinsic Scatter Contribution}

For galaxies in our samples, we obtain averages of $\epsilon_{m}$ within bins of velocity width, shown connected by a solid line, alongside with the measurements of the total scatter in Figure 1. Since measurement and correction errors are independent of whether galaxies are located in clusters or in the field, mean values of $\epsilon_{m}$ are computed from the joint sample of cluster and field galaxies (SCI+SFI), which allows a significant improvement of the statistical reliability of the estimates. We also plot, as dotted lines, the average errors $|b| \epsilon_{x}$ and $\epsilon_{y}$, as defined in paper VI, which represent the total errors on the two variables $x$ and $y$. The sum in quadrature of $|b| \epsilon_{x}$ and $\epsilon_{y}$ approximates but does not match exactly the value of $\epsilon_{m}$, because of the coupling of errors through the inclination correction; on the average, such coupling is mild.

While they constitute an important fraction of the total budget, measurement errors alone cannot fully account for the total amplitude of the scatter about the TF relation. In Figure 1 , we plot as dashed lines the combinations in quadrature of the total measurement error with components of "intrinsic" scatter $\epsilon_{\text {int }}=0.20,0.25$ and $0.30 \mathrm{mag}$, respectively from bottom to top. While the trend in the observed residuals is qualitatively reproduced by these combinations, the slope that results from the assumption of a constant value for the intrinsic scatter does not adequately track the data. It appears instead that while a value of $\epsilon_{\text {int }} \simeq 0.20$ might match the residuals at the high width end, a value as high as $\epsilon_{\text {int }} \simeq 0.35$ might be necessary for low velocity 
widths. The trend in $\epsilon_{i n t}$ that best fits the data is:

$$
\epsilon_{\text {int }}=-0.28 x+0.26
$$

The combination of eqn. (11) and the smoothed trend of $\epsilon_{m}$ is plotted as a solid, heavy line, which matches well the observed trend in the standard deviation of the residuals (filled circles). Because the distribution of residuals about the TF relation exhibits a slight measure of kurtosis, caused by physical and/or spurious outliers, it could be argued that our error model should track somewhere below the standard deviations, and somewhat closer to the mean absolute deviations (crossed squares). We prefer to adopt the conservative approach indicated by eqn. (11), however, with the proviso that the intrinsic component of the TF relation scatter is unlikely to exceed the values predicted by eqn. (11).

A possible contributor to the "intrinsic scatter" component is the spread in distances associated with galaxies in each in cluster sample. Since all galaxies in such a sample are not at the same distance, their spread in distance results in a scatter in magnitudes roughly in the measure $\epsilon_{\text {dist }} \sim 0.10 \theta_{\text {rms }}$ mag, where $\theta_{\text {rms }}$ is a measure of scatter in the sky distribution of galaxies. The average contribution for the clusters in our sample is between 0.10 and $0.15 \mathrm{mag}$, although the TF scatter for nearby, dispersed groups, such as Eridanus, may be dominated by their physical dispersion. We return to this issue in section 5 and in Figures 19 and 20.

TF fits that yield r.m.s. scatter figures smaller than 0.25 mag are likely to be statistical accidents, which can occur when samples are small (e.g. Bernstein et al. 1994 for Coma, Mathewson et al. 1992 for Fornax).

\subsection{Some Implications of the TF Scatter Behavior}

Franx and de Zeeuw (1992) have found that the TF scatter poses strong constraints on the elongation of the gravitational potential in the disk plane of spirals. Our estimate for the "intrinsic" part of the TF scatter reinforces the conclusion of Franx and de Zeeuw, that the average ellipticity of the potential in the plane of the disk must be smaller than about 0.06. On the basis of $2.2 \mu \mathrm{m}$ photometry of 18 face-on spirals, Rix and Zaritsky (1995) find that departures from disk axisymmetry may contribute $\sim 0.15 \mathrm{mag}$ to the TF scatter. Their conclusions apply principally to the inner regions of the disk, which are sampled by the K-band observations, and therefore to TF scatter based on optical observations of the rotation field. When the TF relation is principally based on $21 \mathrm{~cm}$ rotational data, the effect of disk asymmetry on the scatter becomes more ambiguous, as it relates to outer and more poorly mapped regions of disks. Eisenstein and Loeb (1996) estimate that the scatter resulting from varying formation histories of galaxies should exceed $0.3 \mathrm{mag}$ for a broad class of cosmological scenarios. The relatively low values inferred for $\epsilon_{\text {int }}$ suggest either an unexpectedly late epoch of galaxy formation or that a form of secular, 
regularizing feedback mechanism may be responsible for the tightness of the TF relation, as suggested by Silk (1996).

Sandage and collaborators $(1994,1995)$ have advocated a large value of the TF scatter, near or larger than $0.7 \mathrm{mag}$, as an explanation for the high values of the Hubble constant resulting from the use of the $\mathrm{TF}$ relation. If the scatter were as large as proposed by that group, large biases would result, the adequate correction of which would lead to a lowering of the zero-point of the TF template relation, and a consequent reduction of the value of $H_{\circ}$. While the values of the

scatter found by us are not as low as advocated by other groups, it appears unlikely that in the I band the scatter may be as large as suggested by Sandage et al. .

It has been advocated that the use of an inverse fit if the TF relation - one where the "independent" variable is the magnitude rather than the velocity width - does away with the need to correct for incompleteness bias (e.g. Schechter 1980). The nature of the TF scatter, especially the fact that velocity width errors can be overshadowed by other sources, weakens the case for a bias-less inverse TF relation. We return to this issue in section 3.2.

\section{Bias, Morphological and Environmental Corrections}

It has been argued that many of the thorny problems associated with the derivation of a TF relation that arise with field samples are avoided when cluster samples are used. While cluster samples do indeed offer many advantages, they are affected with biases that can severely influence the inferred TF relation. While the optimists are well justified in promoting the adoption of cluster templates over field ones, so are the pessimists in advising skepticism regarding extravagant claims of the curative properties of the cluster elixir. Following seminal work by Roberts, Bottinelli, Gouguenheim, Paturel and Teerikorpi, the discussion of bias in cluster samples' TF has been extensively discussed in the literature, and important points have been underscored in the analyses of Bottinelli et al. (1986), Teerikorpi (1984, 1990, 1993), Kraan-Korteweg et al. (1988), Fouqué et al. (1990), Pierce and Tully (1992), Willick (1994) and Sandage et al. (1995).

Suppose a universal TF relation (UTF) exists. An observed sample can depart from the UTF for a variety of reasons:

(a) If a cluster sample consists of galaxies of various morphologies: do galaxies of all types abide by the same UTF? We consider this question in section 3.1.

(b) An important source of bias results from the coupling of a cluster sample completeness with the scatter in the TF relation. This effect has been recently illustrated in great detail by Sandage and collaborators (Sandage 1994a; Sandage 1994b; Federspiel et al. 1994; Sandage et al. 1995); their graphic approach allows a rapid conceptual grasp of the problem. We discuss this problem and our adopted solution in section 3.2.

(c) Samples extracted from size- or flux-limited catalogs, such as the UGC (Nilson 1973) or the 
CGCG (Zwicky et al. 1961), will in varying degree have built-in biases, which depend on the accuracy of the cataloged photometric quantities which were used to select the sample, rather than on that of the successively measured parameters. In particular, biases can arise from the proximity of sample objects to the catalog limit, and they may be strongly coupled to the amplitude of the scatter in the TF relation. This "edge of catalog" effect can be folded in with the selection function of the sample; in the treatment advocated by Willick (1994), the correction includes that of the incompleteness bias we have discussed above, and becomes the dominant bias correction. We discuss it separately in section 3.3.

(d) When the size of the cluster is not negligible in comparison to its distance, the estimated mean distance to a cluster can be in error. The effect, which is usually quite small, is briefly discussed in section 3.4 .

(e) The so called "homogeneous Malmquist bias" is important in the correction of the estimated TF distances of individual field galaxies, and is computed on the assumption of a Poissonian distribution of galaxies in space. This bias, which couples with the characteristics of the scatter in the TF diagram, is much smaller in the case of clusters than for individual field galaxies. In section 3.5, we derive an estimate for such bias, taking into account the variable character of the scatter in the TF plane, and discuss its implications for the determination of cluster distances.

(f) The cluster environment may affect both the photometric and kinematical characteristics of galaxies, depending on their location in the cluster gravitational potential well. As a result, the location of individual objects in the TF diagram may depend on distance from the cluster center or on the cluster richness. We explore this possibility in section 3.6.

\subsection{TF Dependence on Morphological Type}

The work of Roberts (1978), de Vaucouleurs et al. (1982) and Burstein (1982) raised a serious concern on the predictive quality of the TF technique, by suggesting that a single relation may not apply to all morphological types, a concern expressed by Tully and Fisher (1977) themselves. While it appeared clear that different types follow different TF relations when B band photometry is used, the work of Aaronson and Mould (1983) demonstrated that such dependence becomes negligible when $\mathrm{H}$-band infrared magnitudes are adopted. The question resurfaces in this analysis, for it has not been satisfactorily verified whether a single TF relation is applicable to all types at I band.

The issue is important for us, because the SCI, as any TF cluster sample, spans a broad range of spiral types. This circumstance arises because of the relative paucity of spirals in clusters, as compared with the field. In the field it is feasible to restrict samples to contain only late spiral types (i.e. Sbc and Sc). In clusters, however, the scarcity of spirals induces observers to stretch somewhat the morphological type constraints, in order to accrue statitically meaningful samples. In the overall in+ sample, for example, $7 \%$ of the galaxies are typed Sab or earlier, $30 \%$ are Sb's, 
$57 \%$ are Sbc-Sc's and 6\% are of morphology later than Sc.

Consider Figure 3(a), where we plot with different symbols the location in the TF diagram of SCI galaxies earlier than Sb (large solid circles), Sb's (dotted squares) and later than Sb (small dots). Differences in the behavior of the three classes appear. No sample incompleteness correction (discussed in the next section) has been applied to the data in Figure 3. A fiducial (dashed) TF line of slope -7.68 is superimposed on the data, and it represents the best fit to the types later than Sb. A mild, perhaps monotonically changing dependence of the offset of the TF relation on type is seen; earlier types appear to be slightly fainter, at a given width, than later ones.

We have also investigated possible differences in the behavior of the galaxies later than Sc. These objects are, however, few in number in our cluster sample, and their scatter about the TF relation is typically large. They appear to be somewhat brighter, at a fixed width, than the other types. However the indication among similar objects in the field points in the opposite direction, i.e. they appear to be somewhat fainter than the other types, at fixed width. Given the unclear picture, we thus choose not to fit them separately; in Figure 3, they are included in the late spirals group.

It is well known that galaxies of different type have different distributions of velocity width (Roberts 1978): earlier types are typically faster rotators than later types. The differences suggested by Figure 3(a) could then result from both luminosity shifts or changes in TF slope. In Figure 4(a), we plot solely the galaxies earlier than Sb, combining objects in the cluster sample (SCI; solid symbols) with those in the field sample (SFI), in order to help improve statistical significance. In Figure 4(b), the cluster Sb galaxies are shown. In each of the panels of Figure 4, the dashed line represents the fiducial TF line of slope -7.68 also plotted in Figure 3.

Linear fits to the data in Figure 4 indeed yield shallower slopes than for the later type spirals. However, this impression is tempered by the fact that the early-type spirals cover a narrower range of luminosities and are affected by an incompleteness bias of the type decribed in the next section. The bias produces an apparent reduction of the TF slope, and it may well be stronger for the early type galaxies than for the later types. When the effect of incompleteness is taken into account, the difference in slope between samples of different type becomes statistically insignificant. We thus adopt a morphological type correction which is a plain additive offset:

Types earlier than $\mathrm{Sb}:-0.32 \mathrm{mag}$

Type $S b:-0.10 \mathrm{mag}$

Other types: unchanged

Figure 3(b) displays the same objects as Figure 3(a), except that galaxies of types earlier than Sbc are displaced by the morphological type correction described above.

The type correction adopted for Sb galaxies is only marginally significant. Nonetheless, the $0.10 \mathrm{mag}$ offset is corroborated by inspection of the field galaxy sample; it is also in agreement 
with independent estimates of mass-to-light ratios (Broeils 1992; Vogt 1995).

Early-type spirals occur preferentially in richer clusters, which tend to be more distant in our sample. Since the type correction is more conspicuous for the early types, the concern arises that the type correction may mask a distance bias. We can discount such concern. The type bias is corroborated by the behavior of galaxies in the field sample, as illustrated in Figure 4(a), and by direct comparison of objects of the same average distance but different type.

We close this section with a few comments regarding the issue of linearity of the TF relation. It is interesting to point out that the global TF relation shown in figure 3(a), before any type bias is accounted for, exhibits a mild degree of nonlinearity of the type noted by several authors in the past (i.e. Aaronson et al. 1982; Willick 1990; Pierce and Tully 1996). We have discussed in paper II (see Figure 9 in that ref.) how such nonlinearity can arise in part from the adoption of an inadequate extinction relation. An additional contribution to nonlinearity can result if samples contain a mixture of morphological types, and their differential behavior is ignored. There are obvious advantages of numerical simplicity in the adoption of a linear model. However, it is important to point out that there are no well established physical reasons which demand strict linearity in the TF relation (see Giovanelli 1996).

\subsection{Incompleteness Bias}

Echoing a remark of Sandage et al. (1995), we stress the point that a cluster sample, just because all the galaxies are at roughly the same distance, does not exhibit the properties of a volume limited sample. The parameters of a cluster TF diagram will depend on (a) the degree of completeness with which the luminosity function (LF) is sampled and (b) the amplitude of the scatter in the unbiased UTF. Roberts (1978) first discussed the effect of a Malmquist-like bias on the TF relation, particularly in connection with its application in measuring the value of $H_{\circ}$. Schechter (1980) pointed out that the adoption of an inverse fit, where absolute magnitude is used as the 'independent variable', yields bias-free predictions of distance. An analogous approach has been adopted by several workers (e.g. Pierce and Tully 1992) and discussed extensively (see Teerikorpi 1990, 1993 and references therein). Conversely, the derivation of a large bias correction, based on the assumption of a TF scatter of large amplitude, has been used by Sandage et al. (1995) as a means to explain why previous use of the TF relation may have led to overestimates of the value of $H_{\circ}$.

If samples were selected purely by angular size or flux criteria, and subsequent extinction corrections were negligible, and if furthermore all observational and other correction errors had negligible impact on magnitudes, then the inverse TF relation would indeed yield nearly bias-free

predictions (see, e.g. simulations in paper III). However, since catalogs are inexact, extinction corrections can be large and thus shift points in magnitude, and since errors in corrected absolute magnitude can not only be important but in some circumstances even predominant (e.g. Figure 
2a), the correction for bias becomes an unavoidable chore, whichever fitting convention is adopted. In the following, we describe the nature of the bias, our adopted correction technique and its sensitivity to various relevant parameters.

\subsubsection{The Nature of the Bias}

The nature of the incompleteness bias is dramatized in the simulation shown in Figure 5. A cluster sample is extracted from a population with an LF as shown by the smooth curve plotted along the vertical axis; the sample is however incomplete, as the histogram of magnitudes superimposed on the LF shows. Incompleteness is shown here to have a "soft" edge, indicated by the progressive departure of the histogram from the LF. For a flux limited sample, the histogram would of course track the LF for magnitudes brighter than the limit, then level suddenly to zero. The assumed UTF is represented by a dashed line. In order to dramatize the bias, we have assumed a scatter twice as large as that illustrated by eqn. (3); the assumed scatter in Figure 5 averages about 0.7 mag. A heavy solid line connects filled symbols which identify mean values of the magnitude within bins of velocity width. Incompleteness affects the TF relation derived from the simulated sample in several important ways:

(1) the derived slope is less steep than that of the UTF; the slope becomes progressively shallower as the velocity width diminishes because, due to the incompleteness, low width objects preferentially appear above the UTF;

(2) the zero point is brighter than that of the UTF;

(3) the scatter is underestimated, increasingly so as the velocity width diminishes, because objects with high residuals below the UTF line are missing from the sample.

As a result of this effect, one should expect that uncorrected cluster TF slopes will diminish with increasing distance. This expectation needs to be tempered by noting that the amplitude of the scatter, and thus of the bias, is actually smaller in reality than indicated in the simulation of Figure 5. In spite of the broad variance introduced by the small number of objects per cluster, inspection of Figure 17, which contains TF diagrams of the SCI clusters uncorrected for the incompleteness bias, shows that the steeper TF slopes correspond to Ursa Major and Fornax, two nearby clusters which sample fairly deeply the LF; on the other hand, the distant clusters Coma and A2634, represented by samples which become severely incomplete near $\mathrm{M}_{I}+5 \log h=-20$, have among the shallowest TF slopes. One should also expect the uncorrected TF zero point to become progressively brighter with increasing distance. This effect is small and not discernable in the figures, where zero point variations are dominated by cluster motions. A change in the scatter of individual cluster TF diagrams with distance is also difficult to discern from the plots in Figure 17, due to the smallness of the effect and of the size of individual cluster samples.

In this section we have illustrated the nature of the bias by reasoning within the framework 
of a direct TF relation. Since errors are important in both coordinates, a description of bias could analogously be produced for the inverse relation. As we shall discuss in section 4, our favorite fitting mode will be one that takes however into consideration errors in both coordinates.

\subsubsection{Our Correction Technique. Case study: the Coma Cluster}

Having established the nature of the incompleteness bias, we must decide how to correct for it. Several papers have given correction recipes, mostly attempting to produce analytical or graphic solutions to the problem (Teerikorpi 1993 and references therein; Willick 1994; Sandage et al. 1995). These treatments usually assume a single value for the TF scatter, a sharp apparent magnitude or angular size limit for the completeness of the sample, or both. These conditions are not met by realistic samples. We have emphasized in section 2 that the TF scatter varies significantly with velocity width, mostly driven by measurement errors associated with the velocity widths, disk inclination and correction uncertainties associated with turbulent motions and internal extinction. An inspection of the completeness histograms plotted for each cluster in Figure 10 shows not only the varying degree, but also the softness of the limit of completeness for each cluster sample. Under these circumstances, the incompleteness bias problem is more readily solvable numerically, adopting Monte Carlo techniques. In what follows, we illustrate our correction method, step by step, for the case of the Coma cluster.

Panel (a) of Figure 6 displays the "raw" TF diagram for the in+ sample of Coma. The small unfilled symbols represent the positions of the galaxies as listed in Table 2 of Paper VI. A type correction is applied to galaxies of type earlier than Sbc. These are typically fast rotators. The type-corrected positions are shown as filled symbols and the direct fit to the type-corrected data points as a dashed line. In the same panel, plotted along the left vertical axis is a shaded histogram of the absolute magnitudes of the sample and the LF of the spiral population, which we use for the estimate of sample completeness. Incompleteness sets in near -21 and sampling of the LF ceases below -20 . The estimate of the incompleteness bias requires several ingredients:

(i) a function which describes the sample completeness, $c(y)$;

(ii) a UTF of zero point and slope $\left(a_{u t f}, b_{u t f}\right)$;

(iii) a description of the unbiased TF scatter characteristics.

For each cluster sample, the completeness function is constructed as follows. First we Hanningsmooth (i.e. convolve with a $[0.25,0.50,0.25]$ function) the magnitude histogram, in order to partly reduce the effects of small number statistics. The smoothed histogram is then divided by the LF, producing a histogram $h^{\prime}(y)$. The ratio $h^{\prime}$ is then fit with a function

$$
c(y)=\frac{1}{e^{\left(y-y_{F}\right) / \eta}+1}
$$


(which is the shape of the energy distribution function for the Fermi-Dirac statistics and provides a familiar example of a smooth step function). The parameter $y_{F}$ is the absolute magnitude at which sample completeness approximates $50 \%$ and $\eta$ parametrizes the steepness of the completeness decline. We use the analytical fit to describe completeness, rather than the histogram $h^{\prime}$, in order to reduce the vagaries of small number statistics. Both the histogram $h^{\prime}$ and the function $c(y)$ (dashed line) for Coma are shown in the appropriate panel of Figure 10.

Operationally, the estimate of the incompleteness bias is carried out by producing $n_{\text {times }}$ simulated realizations of the cluster. Each realization has the same distribution of line widths as the real sample. A simulated sample point has a given velocity width parameter $x$ (extracted from the real cluster sample); from it, a value $y=a_{u t f}+b_{u t f} x+\epsilon(x)$ is computed, where $\epsilon(x)$ is the function described by eqn. (3); the point is then passed through a "completeness filter" by comparing the value of the completeness function $c(y)$ for that magnitude with a random number $n_{\text {ran }}$ in the interval $(0,1)$ : if $n_{\text {ran }}<c(y)$ the point is accepted, and if the condition is not met the point is rejected and a new value of $y$ is generated and tested. The simulated sample will reflect the incompleteness characteristics of the real sample; its biases, which can be well estimated if $n_{\text {times }}$ is large, are the most likely estimate of bias for the real sample. The simulated sample is fit by a linear relation $\left(a_{f i t}, b_{f i t}\right)$.

Panel (b) of Figure 6 shows the mean values of the deviation between the simulated sample points and the UTF relation, after binning the data in narrow velocity width intervals and $n_{\text {times }}=800$ trials. This is the incompleteness bias for the Coma cluster.

Panel (c) of Figure 6 shows the average values of the ratio of the r.m.s. scatter computed for the simulated sample, with respect to the TF relation $\left(a_{f i t}, b_{f i t}\right)$, over the "true" value input for the UTF. We appreciate here how the simulated sample underestimates the true scatter of the TF relation at the small width end.

Figure 6(d) is an approximation to an unbiased representation of Coma's TF diagram. To each point in figure 6(a) a correction in $y$ is applied to account for the bias shown in panel (b), i.e. each point is shifted faintwards. The sample is still incomplete, and the scatter at the lower widths is slightly underestimated, but the UTF slope and zero point are recovered free of bias. The dashed line is the same as in panel (a).

Next we investigate the sensitivity of the bias estimate on our input parameters. In Figure 7 we display panels analogous to those in Figure 6, panels (b) and (c), in which the bias is plotted for three different functions describing the scatter $\epsilon(x)$. The solid line shows the bias for our adopted function $\epsilon(x)$, as given in eqn. (3), while the other two lines illustrate the bias if the scatter were respectively 1.5 times and twice as large as the adopted amplitude. In Figure 8, we maintain the scatter function $\epsilon(x)$ fixed at our adopted form, and alter the slope of the faint end of the LF, which affects the completeness function of the sample. Using a Schechter form for the LF, the two cases plotted correspond to exponents of $\alpha=-0.50$ and $\alpha=-0.85$, respectively, for the power law, faint-end part of the functional representation (the value of $\alpha$ used in Figures 
6 and 7 is -0.50). The two functions are shown as an inset in Figure 8b. Finally, in Figure 9, we maintain scatter function $\epsilon(x)$ and LF $(\alpha=-0.50)$ fixed, and change the slope of the UTF between values of -7.0 and -8.0 . It is clear that the main driver of the bias amplitude is the TF scatter. Changes of the UTF slope affect only in minor form the estimate of bias. The slope of the LF, while not affecting in great measure the overall amplitude of the bias, shifts its effects to galaxies of higher velocity width (brighter); as a result, it has an effect on the zero point of the TF relation: in the case of Coma the zero point shift, resulting from a change between the two LF's displayed in Figure 9, amounts to about 0.04 mag.

\subsubsection{Incompleteness Bias Corrections for our Cluster Samples}

In Figures 10 and 11, we summarize the incompleteness bias computations for each of the in + cluster samples in SCI. They were computed using a UTF slope of -7.6, a LF index $\alpha=-0.5$ and a functional description of the scatter amplitude as given by eqn. (3). The coefficients $y_{F}$ and $\eta$ of the completeness function as defined by eqn. (12), computed for each cluster in+ sample, are listed in cols. (2) and (3) of Table 1. Each bias estimate was obtained after $n_{\text {times }}=800$ simulations of the cluster.

In Figure 10, the shaded histograms represent the functions $h^{\prime}$, obtained as discussed in the preceding section. The superimposed dashed lines represent fits of the type described by eqn. (12). The completeness level of $c(y)=1$ has been estimated by eye as shown, compatibly with the noise of the $h^{\prime}$ histograms. The parameters $y_{F}$ and $\eta$ are not computed separately for the in samples. Because the in samples contain typically half the number of objects of the in + samples, their $c(y)$ functions would be quite unreliable. However, for any given cluster the luminosity distributions of in + and in samples are very similar, and it can be safely assumed that the $c(y)$ functions derived for in+ samples are applicable to the in samples as well. The clusters A2197 and A2199, which

are close in the sky and at approximately at the same redshift, are combined in a single sample in Figure 10.

In figure 11, we display the results of our computations for the incompleteness bias for each cluster sample, obtained following the procedure described in detail in the preceding section for the Coma cluster. A solid line superimposed on the results of the simulations indicates the adopted bias correction. Notice how incompleteness affects the TF diagrams of the more distant clusters, such as A2634 and A2197/9, over the whole dynamic range covered by the diagram.

We have also computed the analogous functions to those displayed in Figures 10 and 11, using an exponent $\alpha=-0.85$ for the LF. The corresponding coefficients $y_{F}$ and $\eta$ of the completeness function as defined by eqn. (12) are listed in cols. (4) and (5) of Table 1. We do not show the graphic results (analogous to figs. 10 and 11) for the sake of economy. The changes with respect to the functions displayed in Figure 11 can be garnered by inspection of Figure 8, for Coma. The variance in bias estimates which results from different assumptions of the LF shape will be used in 
section 6 , to investigate the margin of uncertainty on the determination of cluster motion offsets and on the zero point of the TF template relation.

In Figure 12, we plot the bias corrections for each galaxy in the in + samples, as a function of the I-band apparent magnitude. In panel (a), the correction has been computed using a value of $\alpha=-0.5$ for the power law exponent of the LF, while in panel (b) the value $\alpha=-0.85$ was used.

We remind the reader that the bias corrections illustrated here were estimated within the framework of the bivariate TF relation, where a direct fit takes into consideration errors in both the variables, as described in section 4.

\subsection{The Edge- - of-Catalog Bias}

We start with a simple example, which illustrates the concern addressed in this section. Consider the ideal case where a flux-limited sample for a given cluster is extracted from a catalog, so that the sample can be considered complete for all magnitudes $m$ up to a limiting value $m_{l}$. Note that even if the selection were strict, the sample would never be genuinely complete, because the catalog itself will have built-in biases, which can be serious, as discussed extensively by the Cardiff group: see Davies 1994 and references therein. A TF relation with given scatter is used to infer "predicted magnitudes", $m_{t f}$, from velocity widths of each galaxy in the sample. The differences $m_{t f}-m$, when plotted versus $m_{t f}$, will exhibit a positive bias for values near and larger than $m_{l}$ : the scatter in the TF relation shifts some objects to values of $m_{t f}$ larger than $m_{l}$. As in such a sample the number of objects per magnitude bin, $n(m) d m$, rises steeply up to $m_{l}$, the mean estimated distance averaged over $m_{t f}$ bins is biased near $m_{l}$.

Willick (1994) has developed an elaborate mathematical formalism to treat this effect, which can be extended to cases in which samples are not strictly magnitude- or size-limited. For a given cluster sample, for example, he estimates the bias in terms of 5 parameters: the scatter in the TF relation, two so-called "coupling parameters", which relate to the sample selection functions (in magnitude and diameter), and two "closeness parameters", which are estimated for each galaxy from its velocity width, the TF scatter and the size and magnitude limits of the sample. The inclusion of the coupling parameters makes it possible for this approach to treat jointly the edge-of-catalog effect and the incompleteness bias described in the preceding section. However, the incongruous character of each sample's selection functions and the inadequacy of the assumption, made in the derivation of Willick's formulas, that the TF scatter is a constant (see discussion in section 2), raise complications in the application of this technique. We test for the severity of a catalog edge effect in our data in two ways.

First, we reproduce the correction estimated in Willick (1994, his Figure 1b) for a strictly magnitude-limited sample, by means of a Monte Carlo simulation and following procedures similar to those described in section 3.2. We display the results in Figure 13, where we use Willick's nomenclature to define the variables $A=\left(m_{l}-m_{t f}\right) / \sqrt{2} \sigma$ and $B=-\left(m-m_{t f}\right) / \sigma$, where $\sigma$ is 
the scatter in the TF relation, which Willick adopted to be constant. We carried out simulations adopting for $\sigma$ both a constant value and a variable one as described in eqn. (3); in the latter case, its mean value over the simulated sample was adopted in the definition of $A$ and $B$. The results of our simulation for a magnitude-limited sample are represented by the unfilled squares in Figure 13, while Willick's analytical estimate is shown as a continuous curve. When, instead of a sharp magnitude edge, we apply to the simulated sample a completeness function as estimated for our global in+ sample, the expected bias, shown as solid circles, is indistinguishable from zero.

The reason for a lack of an edge-of-catalog bias in our data is that there is no hard edge in our samples at the cataloged magnitude limit; incompleteness starts at significantly brighter magnitudes than either the CGCG (Zwicky et al. 1961) magnitude limit or the Lauberts (1982) limit, and it falls off gradually on the faint end. A corroboration of the lack of an edge-of-catalog bias can be obtained by inspecting the TF residuals as a function of redshift, as shown in Figure 24. Since galaxies near the catalog edge tend to be more distant objects, a catalog edge effect would appear in the form of systematically negative residuals, at high redshift. Such an effect, or any trace of systematicity, are not present in Figure 24.

We thus choose not to apply to our data a correction for the catalog edge bias which, if residually present after the incompleteness bias is applied, appears buried in the noise.

\subsection{Cluster Size}

The assumption that all galaxies in a cluster are at the same distance from us is adequate insofar as the ratio between the cluster physical size and its distance is negligibly small. Such an assumption is increasingly flawed as the distance of the cluster diminishes. There are two kinds of corrections that may need to be applied, in order to account for the extent of clusters along the line of sight. One relates simply to the mismatch which results from working with distance moduli rather than actual distances; this correction is applied to the mean distance of the cluster ensemble. The second needs to be applied to each galaxy, and differs depending on the nearness of the galaxy to the completeness limit of the sample; this correction is related to the incompleteness bias. We consider them separately.

\subsubsection{Cluster Size: Global Bias}

When we fit a cluster TF diagram, we minimize the scatter on the distance modulus, not on the distance. We thus obtain a mean distance modulus which may differ from the mean distance of the cluster, especially if the ratio between diameter and distance of the cluster sample is high. This difference arises because the average of the logarithm is not the logarithm of the average. We estimate this bias by assuming that the distribution of galaxy distance in each sample is Gaussian, with a dispersion which we estimate from the angular spread in the sky. The difference between 
the mean and the true distance modulus can be expressed in terms of the ratio between the size, expressed in terms of the Gaussian dispersion of the galaxy distribution, $\delta$, and the distance $d$ to a cluster: it amounts to $-0.08,-0.06,-0.03,-0.008 \mathrm{mag}$ respectively for $\delta / d 0.20,0.13,0.10,0.05$ radians. For clusters in our sample, the effect is typically small. Estimates of the bias for each sample, expressed in magnitudes, are given in cols. (6) and (7) of Table 1, respectively for the in and in + samples of each cluster.

\subsubsection{Cluster Size-Sample Incompleteness Bias}

Let the distribution of galaxy distances within a cluster be Gaussian, with a dispersion which can be translated to $\sigma_{d}$ mag e.g., if the dispersion in distances along the line of sight is $10 \%$ of the mean distance, $\sigma_{d}=0.21$ mag. When the assumption is made that all galaxies in the cluster are at the same distance from us, the adopted absolute magnitude $y$ of a given galaxy will be off the true value according to a Gaussian probability of dispersion $\sigma_{d}$. If the sample is complete at magnitude $y$, the mean expectation value of $y$ will be equal to the true value, i.e. the probability that the galaxy is in the foreground half of the cluster is equal to that of finding the galaxy in the background half. However, if the sample completeness decreases steeply near $y$, it will be more likely that the galaxy be placed in the foreground half than in the background half of the cluster. Assigning to the galaxy the mean distance modulus of the cluster will lead to an overestimate of its luminosity.

The effect described is illustrated in Figure 14. The curve labelled $c(y)$ is the completeness function of the sample, as parametrized by eqn. (12); the curve labelled $E(y)$ describes the Gaussian extent of the cluster, whereby galaxies of absolute magnitude $y_{\circ}$ are seen spread with dispersion $\sigma_{d}$ (for the computation of this bias we assume that a Gaussian distribution in size translates into a Gaussian distribution in distance modulus; the marginal validity of this assumption affects the bias estimates negligibly, only in the second order); the heavy-traced curved is the product $c(y) E(y)$. The bias that arises from the coupling of cluster size and sample incompleteness is the difference between the centroid of $c(y) E(y)$ and that of $E(y)$, i.e.

$$
\beta_{E I}\left(y_{\circ}\right)=\bar{y}-y_{\circ}
$$

where

$$
\bar{y}=\int y c(y) E(y) d y / \int c(y) E(y) d y
$$

Quantitatively, the effect is generally small. For example, in the case of a cluster with a line of sight extent equal to $10 \%$ of its distance, and a completeness function of the type given by eqn. (12) with $\eta=0.5 \mathrm{mag}$, the bias at $y_{\circ} \simeq M_{F}$ is $\beta_{E I} \simeq-0.05 \mathrm{mag}$. 
The correction $\beta_{E I}$ should in principle be applied only to cluster galaxies, i.e. objects in the in samples, for which the assumption of a common distance was made. Galaxies in the in + samples were assigned the distance derived from their individual redshift, and such a bias correction would appear at first not to be necessary. However, a measure of scatter about their velocities should be expected for the motions of field galaxies as well. Such scatter is equivalent to describing the distance by a probability distribution, centered about that corresponding to the redshift velocity, which will be present even in the absence of large scale velocity flows. A bias similar to that described above for cluster galaxies thus arises, when the absolute magnitude of the galaxy approaches values for which the sample is incomplete. For the non cluster galaxies pertaining to the in + samples, we can thus compute a bias similar to $\beta_{E I}$, where the function $E(y)$ is assumed to be a Gaussian of dispersion $\sigma_{d}=5 \log \left(1+\sigma_{v} / c z\right)$, with $\sigma_{v} \simeq 200 \mathrm{~km} \mathrm{~s}^{-1}$.

\subsection{Homogeneous Malmquist Bias}

The so-called Malmquist bias produces statistical underestimates of TF distances. This arises from the fact that within a given solid angle, the number of galaxies between distances $r$ and $r+d r$ usually rises with $r$. Then, for galaxies of estimated distance modulus $\mu_{e} \pm \Delta_{\mu}$, the most probable distance is not $r_{e}=10^{0.2\left(\mu_{e}-c\right)}$ (where $c$ is the usual scaling term which depends on the adopted units of distance), but a value $r>r_{e}$, because there are more galaxies between $\mu_{e}+\Delta_{\mu}$ than between $\mu_{e}-\Delta_{\mu}$. When the assumption is made that the space distribution of galaxies is Poissonian, the qualification of "homogeneous Malmquist bias" is used. In that case,

$$
r=r_{e} e^{3.5 \Delta^{2}}
$$

where $\Delta=10^{0.2 \Delta_{\mu}}-1$ is the relative error in the distance estimate arising from $\Delta_{\mu}$. For example, for a scatter of $\Delta_{\mu}=0.3 \mathrm{mag}$, the distance relative error is $15 \%$, or $\Delta=0.15$. For recent reanalyses of this classical problem (Malmquist 1924), relevant to the measure of galaxy distances, see Lynden-Bell et al. (1988), Feast (1987), Landy and Szalay (1992), Sandage (1994), our Paper III and references therein. The estimate of the bias in a clustering regime is very different from the homogeneous case and will depend on the characteristics of the space density field.

To the extent that clusters can be characterized as single objects in space, a Malmquist bias correction (MBC) in principle also applies to their estimated distances. However, since the distance modulus determination of a cluster is more accurate than that of a single galaxy (roughly by a factor $N^{1 / 2}$, where $N$ is the number of galaxies measured in the cluster), the amplitude of the $\mathrm{MBC}$ is quite small.

Our analysis of the error budget of the TF relation shows that $\Delta_{\mu}$ depends on the galaxy velocity width, and thus so will the Malmquist bias. In Figure 15 we show the homogeneous MBC, $r / r_{e}$, computed assuming that the TF scatter, and therefore the uncertainty on the distance 
modulus of a galaxy, behaves according to eqn. (3). The change in $r / r_{e}$ as a function of $x$ is indicated by the solid line. The homogeneous MBCs that would be obtained adopting fixed values of $\Delta_{\mu}$ of, respectively, $0.25,0.35$ and $0.45 \mathrm{mag}$, are shown by dashed lines.

For individual galaxies the homogeneous MBC varies typically between 1.05 and 1.20 , respectively for galaxies on the high and on the low end of the velocity width distribution. Note how the adoption of a constant value for $\Delta_{\mu}$ can lead to gross under- or overestimates of the MBC. For example, consider a fast rotator with $\log W=2.75$, which will also be a bright galaxy likely to be observed even at relatively large distance. The homogeneous MBC for such an object, if measurement errors were typical, would be $r / r_{e} \simeq 1.04$. On the other hand, the adoption of a constant value for the TF scatter of $0.35 \mathrm{mag}$ (the mean value for our cluster sample), would lead to a correction of $r / r_{e} \simeq 1.11$. If the galaxy were at rest at, say $c z=5000 \mathrm{~km} \mathrm{~s}^{-1}$, the difference in the estimated redshift distance resulting from the two corrections would be greater than 300 $\mathrm{km} \mathrm{s}^{-1}$ : the flawed (constant $\Delta_{\mu}$ ) MBC would produce the equivalent of a large, negative and spurious peculiar velocity.

For clusters, the relative error on the distance, $\Delta$, is roughly $N^{1 / 2}$ times smaller than for individual galaxies. As a result, except for very small samples, the homogeneous MBC would not exceed 1.01. We will consider the impact of this small correction in our analysis of cluster motions in Paper VIII.

\subsection{Effect of Cluster Environment}

Galaxies seen projected near cluster cores are likely to suffer disruptive interactions with the cluster environment. It is therefore necessary to verify that the same UTF is satisfied by those objects, as that representative of peripheral objects and field galaxies. For that purpose, we perform a simple test. After corrections for morphological type and incompleteness bias are applied, and after the offsets associated with cluster motions are applied as discussed in section 6.1, the magnitude residuals with respect to a direct TF relation of coefficients $(-21.01,-7.68)$ are calculated. In figure 16, those residuals are plotted versus the projected distance from cluster centers. In panel (a), all galaxies in the in + samples are included, while in panels (b) and (c) galaxies have been separated between the 12 higher density clusters (A262, A400, Cancer, Hydra, A1367, Cen30, Coma, ESO508, A3574, A2197/9, A2634 and A2666) and the remaining 11 lower density clusters, respectively. There is no apparent trend in the residuals, and we judge that there is no sufficient reason to doubt that a single UTF is satisfied by the entire sample.

It should be noted that a dependence on cluster environment of TF parameters may already have been removed from the data in Figure 16, in the form of the morphological type correction. Because of the morphology-density relation, earlier spiral types are likely to be found in closer projection to the cluster core than late spiral types. It appears however that the type correction discussed in section 3.1 applies to early type spirals that are not found in clusters as well (see 
Figure 4a), so the type correction cannot be attributed to the effect of the cluster environment.

\section{Fitting Procedures}

For a set of $N$ data points $\left(x_{i}, y_{i}\right)$, we determine "direct", "inverse" and "bivariate" forms of the linear TF relation, as well as a quadratic model, according to the following rules.

The direct fit consists in the determination of the coefficients $a_{d i r}$ and $b_{d i r}$ in the form

$$
y(x)=a_{d i r}+b_{d i r} x
$$

via minimization of the merit function

$$
\chi^{2}=\Sigma_{i=1}^{N}\left[\frac{y_{i}-y\left(x_{i} ; a_{d i r}, b_{d i r}\right)}{\epsilon_{i}}\right]^{2}
$$

where we adopt $\epsilon_{i}=\sqrt{\epsilon_{y, i}^{2}+\epsilon_{\text {int }}^{2}}$, the total uncertainty in $y$ arising from both measurement and intrinsic errors.

The inverse fit refers to the determination of $a_{i n v}$ and $b_{i n v}$ in

$$
x(y)=-a_{i n v} / b_{i n v}+y / b_{i n v}
$$

where the merit function to be minimized is analogous to that of the direct case, except for an exchange of $x$ with $y$, and the assignment $\epsilon_{i}=\sqrt{\epsilon_{x, i}^{2}+\left(\epsilon_{\text {int }} / b\right)^{2}}$.

In the bivariate case, errors in both $x$ and $y$ are taken into consideration, and we fit

$$
y(x)=a_{b i}+b_{b i} x
$$

where the error used in the computation of the merit function by means of eqn. (16) is defined as $\epsilon_{i}=\left[\left(\epsilon_{x, i} b_{b i}\right)^{2}+\epsilon_{y, i}^{2}+\epsilon_{\text {int }}^{2}\right]^{1 / 2}$.

The three sets of coefficients $\left(a_{d i r}, b_{d i r}\right),\left(a_{i n v}, b_{i n v}\right)$ and $\left(a_{b i}, b_{b i}\right)$ are computed for each subsample, separately for each cluster or group. For the three methods the amplitude of the average r.m.s. scatter figure is estimated via

$$
\sigma=\sqrt{\chi_{\min }^{2} / \Sigma_{i}\left(1 / \epsilon_{i}^{2}\right)}
$$


where $\chi_{\min }^{2}$ is the minimum of the appropriate $\chi^{2}$, i.e. that which corresponds to the chosen solution $\left(a_{d i r}, b_{d i r}\right),\left(a_{i n v}, b_{i n v}\right)$ or $\left(a_{b i}, b_{b i}\right)$. For the bivariate fit, we also estimate $\sigma_{a b s}$, the mean absolute deviation in $y$.

For the global sets resulting from the combination of all the cluster samples, discussed in section 6 , we also estimate the most likely slope of the "primitive" linear TF relation which the data set would follow in the absence of scatter. We do so by producing a large number of simulated samples, fitting each as we do for the real data sample, and keeping track of the fit parameters. For each simulated sample, we maintain the values of the linewidths of the real sample, and extract random deviates of a population having the completeness characteristics and the average scatter behavior described in section 2. We loop through a set of simulated TF slopes, and called the "primitive" slope that which yields the closest approximation to the parameters fit to the real data. This value usually tracks closely the slope of the bivariate fit described above.

To our global sets we will also fit a quadratic relation of the form

$$
y(x)=c_{\circ}+c_{1} x+c_{2} x^{2}
$$

where the coefficients $c_{i}$ are obtained by minimizing a merit function similar to that in eqn. (16).

\section{Individual Cluster TF Relations}

Figures 17 and 18 show the TF diagrams for galaxies in in samples of each cluster. In Figure 17, filled circles identify galaxies in the in samples; the data points in this figure are not corrected for any of the biases discussed in section 3. In Figure 18, the location of data points is corrected for incompleteness and morphological type bias, as discussed in sections 3.1 and 3.2, and symbols are replaced by error bars. The error bars refer to the "measurement errors" $\epsilon_{x}$ and $\epsilon_{y}$ as defined in section 2. The same fiducial line of slope -7.6 is plotted in each panel of Figures 17 and 18 .

In distant clusters, we naturally tend to sample the more luminous galaxies. The completeness functions of all clusters, discussed in section 3.2, are shown in Figure 10. Significant offsets with respect to the fiducial line are clearly exhibited in Figure 18 by Eridanus, Ursa Major, ESO508 and MDL59; these are among the nearest clusters in our set. Their samples tend to reach fainter in the LF. Because the vertical displacements produced by motion are largest and prevalently faintwards for these clusters, their magnitude distributions appear to reach even deeper than they do in reality. The completeness functions shown in Figure 10 have been estimated after a shift that accounts for each cluster's motion was applied. These shifts are discussed in section 6.1.

In Table 2 , for each cluster we list the three sets of fit coefficients $\left(a_{d i r}, b_{d i r}\right),\left(a_{i n v}, b_{i n v}\right)$ and $\left(a_{b i}, b_{b i}\right)$ as defined in the preceding section. The direct and bivariate linear least squares fits are applied to the bias-corrected cluster samples shown in Figure 18. The inverse fits are applied to 
the cluster samples without a correction for incompleteness bias. We also list estimated mean uncertainties on $\left(a_{b i}, b_{b i}\right)$, and two estimates of the average scatter: $\sigma_{b i}$ (col. 11), the scatter with respect to the bivariate relation, and $\sigma_{a b s}$ (col. 12), which is the scatter in the values of the absolute deviation about $\left(a_{b i}, b_{b i}\right)$. The number of objects used to fit each sample, $N$, is listed in col. (2) of Table 2 .

It should be kept in mind that an appropriate inverse fit should require the estimate of an incompleteness bias, which will be different from the one applied for the direct and bivariate fits, as we have discussed in section 3.2. We have not estimated that version of the bias, and include the inverse fits estimated without a bias correction purely for comparison with inverse fits in other sources, which usually ignore the incompleteness bias as well.

As mentioned before, all galaxies comprising the in samples were assigned the same distance, that obtained from the cluster systemic redshift. This assignment was made on the basis of angular

and kinematical information which is not completely unambiguous. It is possible that some of the objects assigned cluster membership are decoupled from the virialized cores and partake of pure Hubble flow. If a significant fraction of galaxies in our samples suffered from that misclassification, an important bias could be introduced in both the individual cluster TF relation and in the global template to be constructed by merging all cluster samples. This bias would result because, especially for more distant clusters, interlopers in the foreground would more often be included than those in the background. In Figure 19 we display the magnitude residuals from a TF relation of slope -7.6, after forcing the mean residual to be zero for each cluster sample. This is done for each of the in cluster samples. Residuals are plotted versus the logarithm of the redshift of each galaxy, to which the logarithm of the systemic redshift of the cluster is subtracted, so that in each plot the center of the cluster corresponds to the point $(0,0)$. Galaxies which are freely expanding with Hubble flow but were erroneously assigned cluster membership would be found to scatter about a line of slope 5, as indicated by the dashed line, in Figure 19. Any preferential alignment of data points with that line would indicate contamination of the cluster sample with interlopers. While it may be possible to question a few data points, by and large the cluster diagrams yield little evidence of any systematic contamination. Fig. 20 displays all in samples merged together in a similar representation, illustrating that no significant forward-backward interloper bias affects globally our in samples.

\section{TF Template Relation}

The individual, bias-corrected TF data sets of each cluster can be combined to obtain a TF template relation. We will refer to the result of the combination as the global set. We first describe the procedure adopted in carrying out the cluster combination. We then derive the parameters that best describe the template relation fit to the global set and discuss the extent to which it approximates a desired UTF. 


\subsection{Cluster Combination}

For inclusion in the global sample, the absolute magnitude of the $j$-th galaxy in the $k$-th cluster is corrected in the mode

$$
y_{j, k}^{c}=y_{j, k}+\beta_{t y p}\left(\operatorname{type}_{j, k}\right)+\beta_{i n c, k}\left(x_{j, k}\right)-\Delta y_{k}
$$

where $y_{j, k}$ is the magnitude listed in Table 2 of Paper VI, $\beta_{t y p}$ is the morphological type correction described in section 3.1, $\beta_{i n c, k}$ is the incompleteness bias shown in Figure 10, which is a different function for each cluster, and $\Delta y_{k}$ is an offset, also specific to each cluster sample. The only ingredient we have not discussed so far is the offset $\Delta y_{k}$. This set of parameters is introduced to represent the motion of each cluster, with respect to the reference frame defined by the template relation.

For a linear TF of slope $b$, the TF zero point of the cluster $k$ will be $a_{k}=a_{\circ}+\Delta y_{k}$, where $a_{\circ}$ is a constant. We simultaneously determine $b, a_{\circ}$ and the set of offsets $\Delta y_{k}$ by minimizing the quantity

$$
\chi^{2}=\Sigma_{j, k}\left[\frac{y_{j, k}^{c}-a_{\circ}-b x_{j, k}}{\epsilon_{j, k}}\right]^{2}
$$

with the condition that

$$
\Sigma_{k} N_{k} \Delta y_{k} / \Sigma_{k} N_{k}=0
$$

where the sum is intended over a subset of "distant" clusters, the choice of which is discussed in the next section, and $N_{k}$ is the number of galaxies for the $k$-th cluster sample. The errors $\epsilon_{j, k}$ are as defined after eqn. (18). In the case of a quadratic fit (eqn. 20), a similar minimization process is followed, with the difference that an additional coefficient is simulataneously determined.

Eqn. (23) defines our "inertial frame", i.e. it implies that the set of "distant" clusters involved in the summation defines a mean null velocity with respect to the CMB. We return to this point in section 6.2 .

In Table 3, we list values of $\Delta y$ for each cluster sample: in cols. (3-5) after linear TF fits and in cols. $(6,7)$ after quadratic fits. In col. (3), the offsets $\Delta y$ are computed after applying a correction for morphological type, but not one for incompleteness bias, to the data. In cols. (4) and (5) the incompleteness bias correction is diversely applied: in col. (4), it is computed assuming an LF with a power law exponent $\alpha=-0.50$, while the value adopted for the computation of offsets in col. (5) is $\alpha=-0.85$ (see section 3.1.1 and Figure 8). In cols. (6) and (7), offsets were computed after fitting a quadratic TF relation; incompleteness bias corrections for columns (6) and (7) are the same as adopted for cols. (4) and (5), respectively. An estimate of the uncertainty 
of the last two figures of each value is given between brackets, for the values tabulated in col. (4); similar uncertainties apply to each column. The uncertainties were estimated by taking the largest number between the mean r.m.s. scatter of the fit to the cluster sample, $\sigma_{b i}$ (tabulated in col. 11 of Table 2) and a value of $0.35 \mathrm{mag}$, and dividing that value by the square root of the number of objects in the given cluster sample. Uncertainties in the cluster systemic redshift were not included in this estimate. The values listed in Table 3 will be used in paper VIII in the analysis of the cluster peculiar velocity field.

The global sample obtained by combining individual cluster samples as described by eqn. (21) yields a TF relation of slope and zero point that are corrected for incompleteness and morphological type bias and for the motions of individual clusters, to the extent that the "distant" cluster subset can be assumed to yield a null mean velocity with respect to the CMB. Because the global sample is the sum of many incomplete cluster samples, it does not populate the TF plane in the same manner that a complete, volume limited sample would. While its slope and zero point should be expected to mimick those of an unbiased sample with the same sky and velocity distribution characteristics, its measured scatter will underestimate that of an unbiased sample, for the reasons discussed in section 3.2.2 and illustrated in Figure 6(c). We will discuss that effect in a more quantitative way in section 6.3.

\subsection{The Cluster Reference Frame}

The bias corrections that were applied to each of the cluster samples help produce a global template with a slope which is as close as we are likely to get to that of the "primitive" UTF. Ideally we would wish the zero point to identify with that of a reference frame at rest with respect to the CMB. Since the zero point is specified in magnitudes, and since it is fair to assume that the cluster peculiar velocity distribution function does not depend on geocentric distance, the TF zero point is best defined if the cluster set used to specify it is a distant one: for $V_{\text {pec }} / c z \ll 1$, the corresponding magnitude offset is $\Delta m \simeq-2.17 V_{\text {pec }} / c z$.

Let the cluster velocity distribution function be characterized by a r.m.s. one-dimensional peculiar velocity $<V_{p e c}^{2}>^{1 / 2}$. Then the average of the velocities of $n$ randomly chosen clusters, placed at a mean redshift $\langle c z\rangle$, would yield a most probable magnitude offset from the UTF of

$$
|\Delta m|_{\text {exp }} \sim 2.17<V_{\text {pec }}^{2}>^{1 / 2}<c z>^{-1} N^{-1 / 2}
$$

Bahcall and Oh (1996) report a value of $\left\langle V_{\text {pec }}^{2}>^{1 / 2}=293 \pm 28 \mathrm{~km} \mathrm{~s}^{-1}\right.$. For $N=16$ and $<c z>=6000 \mathrm{~km} \mathrm{~s}^{-1},|\Delta m|_{\text {exp }} \sim 0.03$ mag. Since clusters in our sample are not randomly distributed, e.g. N383, N507 and A262 are in the Perseus-Pisces supercluster, A1367 and Coma are both in the Coma supercluster, A2197 and A2199 are similarly associated, etc., the actual number of clusters with uncorrelated velocities is less than 24 . In addition, it is wise to remove 
those clusters that are at $c z<3000 \mathrm{~km} \mathrm{~s}^{-1}$ or that exhibit large motions, from the set that will be used to determine the TF zero point, in order to reduce the kurtosis of the distribution of magnitude offsets. In Table 1, we have labelled with an asterisk the 14 clusters that have been chosen to define the TF zero point. Their mean redshift is about $6,000 \mathrm{~km} \mathrm{~s}^{-1}$ and we shall assume conservatively that they represent about 9 independent large scale aggregates.

A set of cluster galaxies will allow the definition of a TF template with a statistical accuracy that improves with the number of objects involved in the TF relation. For example, for a mean scatter of about $0.35 \mathrm{mag}$, the sample of about 250 in galaxies in the combined 14 reference clusters will yield a TF zero point with a statistical error of 0.03 mag. This is independent on whether the cluster set represents a system globally at rest with respect to the CMB or not. Using eqn. (23), we can estimate that the reference cluster sample approximates the TF zero point, identifying rest with respect to the $\mathrm{CMB}$, to within approximately $\sim 0.035 \mathrm{mag}$. Combining these two figures in quadrature, we obtain an expectation of kinematic zero point accuracy for the global in sample of $0.045 \mathrm{mag}$. For the in + sample, the expectation improves slightly, to 0.040 mag. The sparse number and uneven sky coverage of the distant cluster samples advises caution, however, suggesting that somewhat larger uncertainties may apply. We expand on this analysis in Paper VIII.

\section{3. $\quad$ Fits to Cluster Templates}

Fig. 21(a) displays the global template obtained by combining the in cluster samples. In Figure 21(b) the analogous combination is displayed for the in + samples and in Figure 21(c) we show only the measurement error bars $\left(\epsilon_{x}, \epsilon_{y}\right)$ for the objects in Figure 21(b).

In Table 4 we give the results of linear fits to the global sets. First, we display the results for the global in and in+ sets, without correcting cluster samples for the incompleteness bias; the fit parameters are given by the first two rows of the Table. Then we display fits to several subsets of bias-corrected samples, separately for the two cases in which the bias computation was carried out using a faint-end power law exponent of the luminosity function $\alpha=-0.50$ and $\alpha=-0.85$. For each value of $\alpha$ we fit several subsets of the data, as described in col. (1) of Table 4, thresholding the data by inclination, type, velocity width and $2.5 \sigma$-clipping. In each case, we also estimate the most likely slope of the "primitive" TF relation which would yield the given set of fits, $b_{p}$, listed in col. (13). As mentioned in section 4, the primitive relation is usually well approximated by the bivariate fit. The formal errors on the slope of the fits are typically on the order of 0.15 , or $2 \%$, while those on the zero point are on the order of 0.02 mag. For the bias-corrected samples, no inverse fits are computed.

Among the fits to data subsets in Table 4, the ones to objects of type Sb and earlier yield significantly lower slopes than the other subsets. This may be an indication of the fact that our

type correction discussed in section 3.1 is inadequate. As we discussed in that section, however, the 
completeness of early type objects may differ from that of those of later type, and the difference in slope may partly reflect that aspect. As the number of early type objects included in TF samples increases, it will be possible to assess the character of the applicable internal extinction laws, TF relations, etc. For the moment, we choose to apply the simplest of corrections, as indicated in section 3.1.

As expected, the correction for the incompleteness bias dims the zero point and steepens the TF relation. The effect is stronger as the value of the LF exponent $|\alpha|$ increases, because that implies that samples become progressively more incomplete, and thus require a larger bias correction.

The in samples exhibit a slightly steeper slope than the in + sample, but the difference is of marginal significance at best.

It is also useful to point out that the cluster offsets $\Delta y$ are not very sensitive to the choice of the adopted LF, or even to whether the incompleteness bias correction is applied at all. That is because the cluster offsets $\Delta y$ are affected by the relative differences of the incompleteness bias corrections, averaged over all galaxies in a cluster sample, rather than by their actual amplitudes.

Finally, in Table 5 we give fit coefficients for the quadratic relation (20), for a more restricted set of samples. The statistical significance of the difference between the linear and quadratic fits is marginal for the cluster set. However, it appears that further inclusion of dwarf and irregular galaxies, extracted from the low luminosity end of the LF, would drive the TF slope steeper and introduce noticeable nonlinearity (Hoffman and Salpeter 1996). While the use of such objects on peculiar velocity studies is of very limited usefulness, due to the large associated scatter, a sample which includes them would be poorly served by a strictly linear TF relation.

\subsection{Underestimate of Scatter in the Template}

In section 3.1 we discussed how the incompleteness bias affects not only the slope and zero point of the TF relation, but also its measured scatter. The effect is one of reducing the apparent scatter near the faint end of the TF relation, as shown in Figure 6(c). Before combining cluster sets, each galaxy is shifted by the incompleteness bias correction, which produces a TF relation in which bias for the slope and zero points have been formally removed. The scatter, however, remains underestimated. The range of velocity widths over which the underestimate occurs varies from cluster to cluster, shifting to lower widths for the more nearby clusters. When the clusters are combined to obtain the global set, mild underestimates of the scatter result throughout the lower half of the velocity width range. We have obtained a Monte Carlo simulation of the underestimate, by producing the combination of the cluster samples, simulated according to the incompleteness characteristics of each as described in section 3, and repeating the simulation a large number of times (800 in our case). Figure 22 shows the results of this simulation, and illustrates that the underestimate is quite mild. If we fit the data in Figure 21(b) and measure the scatter as a 
function of velocity widths, that measure is always at least $90 \%$ as large as the real scatter that would result if we had dealt with complete samples. Thus, the average, measured scatter values tabulated in Table 3 underestimate the true scatter by a small amount. The bias corrected values of the TF scatter, for each tabulated sample, exceed those measured by about $0.02 \mathrm{mag}$, and are not tabulated separately. The bias correction was however taken into account in section 2 , in the analysis of the properties of the scatter in the TF plane (see fig. 1). The smooth line fitted to the simulation data in Figure 22, which parametrizes the underestimate of the scatter as a function of velocity width, was used to correct the location of the unfilled symbols in Figure 1 to that of the filled circles and crossed squares, which represent estimates of the bias-corrected total scatter of the TF relation.

\section{TF Residuals}

A simple test of adequacy of a TF template relation consists in the investigation of the behavior of residuals. Systematic trends in the amplitude and scatter in the residuals have already been used in section 2 (Fig. 4), to obtain an understanding of the components in the TF relation error budget; in section 3.1 (Figs. 5 and 6) to infer morphological type differences in TF offset and slope; in section 5 (Figs. 19 and 20) to infer that cluster samples are not seriously contaminated by fore- and background objects; and in section 3.3 (Fig. 24) to conclude that no catalog edge bias effects are present in our data. The only significant evidence for a trend in the residual distribution was noted in the their dependence on morphological type, which was subsequently modelled and resulted in a morhological type correction to the data.

In Fig. 23, we plot the magnitude residuals of individual galaxies in the in + global sample, versus angular size (panel $a$ ) and apparent magnitude (panel $b$ ). The residuals are computed with respect to the bivariate fit of slope -7.68 . We plot individual galaxies as small unfilled triangles and running averages as large, solid circles. We note that for intermediate values of the angular size, or of the magnitude, residuals are flat and there is no trace of a trend. However, at the two ends of the distributions residuals deviate slightly from zero, on the average. At first, this may be thought of as the signature of a problem in our treatment, while in reality the observed weak deviation of the average deviations is exactly what should be expected. Consider the simulated cluster sample shown in figure 5. The few faintest galaxies in the sample tend to be below the TF line, and the few brightest tend to be above the TF line; the amount by which this deviation occurs depends on the amplitude of the TF scatter. The effect is greatly reduced (but not cancelled) if residuals are estimated with respect to the inverse fit. For every cluster sample, the faint end occurs at different absolute magnitudes, but generally at the same apparent magnitude, so that the cluster combination does not cancel the effect. Simulations of the effect show that the expected amplitude of the main residuals should be about as large as observed.

In Figure 24(a), we show the residuals with respect to the above mentioned bivariate relation, plotted versus the redshift of each individual galaxy (rather than that of the cluster as a whole, 
thus reducing the degree of crowding of the data points in the plot). Reassuringly, the average residual remains null through the whole redshift range. In Figure 24(b), residuals are plotted versus disk inclination angle to the line of sight, verifying that the adopted correction schemes which depend on inclination are adequate.

It appears that no significant, unexpected biases, related with observed parameters of sample objects, affect our global cluster set.

\section{Summary}

We have presented I band photometry and spectroscopic data of 782 spiral galaxies in 24 clusters of galaxies, garnered from our own observations and from material in the public domain. Of those, 555 galaxies are cluster members or lie close enough to the clusters' peripheries, to be useful in a study geared at obtaining a TF template relation. We have divided the galaxies in two groups: an in group of 360 galaxies, thought to be bona fide cluster members, and an in + which includes in addition objects that are peripheral to the clusters.

We analyzed in detail the characteristics of the scatter in the TF relation. The average total scatter is about $0.35 \mathrm{mag}$; its amplitude however varies between about 0.25 mag among fast rotators and more than $0.40 \mathrm{mag}$ among the slowest rotators in our sample. Measurement errors contribute to this trend, in the sense that they tend to be globally larger for less luminous, slow rotators. However, we found that uncertainties associated with measurements and with corrections applied to the observed parameters cannot fully account for the total observed scatter. An additional component, which we refer to as "cosmic" or "intrinsic", is necessary. This component may result from a variety of sources, such as asymmetries in the spiral disks' light and velocity fields and differences in the formation histories of galaxies. The intrinsic scatter component varies with galaxy luminosity, or velocity width, being larger — perhaps as large as 0.35 mag — for the slowest rotators. The intrinsic scatter among the most luminous, fastest rotators may be 0.20 mag or even smaller. We found that the systematically changing character of the TF scatter has important effects in the estimate of bias corrections, and that the assumption of constant values for the scatter can easily lead to spurious results. In addition, the variety of sources of scatter weakens the merits of adopting the inverse form of the TF relation as a bias-free device.

We have discussed in detail the impact of galaxy morphology, environment and bias on the TF relation.

We found that, even when I band photometry is utilized, galaxies exhibit differences in their TF properties that correlate with morphological type. A correction is necessary to galaxies of earlier spiral types, in order to make their use compatible with that of the Sbc and Sc galaxies that form the bulk of our sample. We have used the simplest of corrections, that of a simple additive term, in order to bring the earlier types to consistency with the later ones. This is a conservative choice that may turn out to be inadequate. With varying type, it is quite possible 
that variations in the light-to-mass ratios and internal extinction properties may lead to changes in the TF slope. Since the number of objects of type earlier than Sb in our sample is relatively small, and the behavior of Sb galaxies appears only marginally different from that of later types, we have chosen not to apply more elaborate correction schemes until the size of TF samples of earlier types increases, and independent determinations of mean properties become more reliable.

The effect of incompleteness was analyzed next, and a Monte Carlo technique was described for the computation of the associated bias. We discussed the sensitivity of the bias estimates on the scatter of the TF relation, the shape of the luminosity function of the galaxy population and the slope of the TF relation. An accurate determination of the scatter properties is necessary for a reliable estimate of the bias, as the scatter amplitude is its main driver.

We then analyzed other possible sources of bias, namely: (a) that arising from the nearness of sample galaxies to the limit of the catalog from which the objects are extracted, which in our case is negligible; (b) the effect of cluster depth along the line of sight, also a very minor effect; (c) the dependence of TF properties on galaxy location in the cluster, which we found to be undetectable in our sample. Lastly, we considered the homogeneous Malmquist bias. This effect is relatively unimportant for cluster samples but it can make substantial corrections necessary for individual galaxy distances. In general, it is modulated by the density function of the galaxy distribution and it is most often applied as an 'inhomogeneous' Malmquist bias correction. In either case, we found that the Malmquist bias amplitude is strongly affected by the variable character of the TF scatter, and that the application of the correction in its 'standard' form can lead to grossly deviant estimates of a galaxy's TF distance.

Finally, we obtained a global template TF relation by combining the bias-corrected TF data of the 24 clusters. In the process, we estimated relative offsets of each cluster from the reference frame formed by the set of the 14 most distant clusters, which defines the best estimate of null velocity with respect to the cosmic microwave background radiation field that can be garnered from our sample.

The TF relations thus obtained will be utilized elsewhere in the analysis of the cluster motions and of the peculiar velocity field of individual field galaxies.

It is a pleasure to thank Dr. M. S. Roberts for carefully reading this paper and making a number of suggestions that improved it substantially. The results presented in this paper are based on observations carried out at the Arecibo Observatory, which is part of the National Astronomy and Ionosphere Center (NAIC), at Green Bank, which is part of the National Radio Astronomy Observatory (NRAO), at the Kitt Peak National Observatory (KPNO), the Cerro Tololo Interamerican Observatory (CTIO), the Palomar Observatory (PO), the Observatory of Paris at Nançay and the Michigan-Dartmouth-MIT Observatory (MDM). NAIC is operated by Cornell University, NRAO by Associated Universities, Inc., KPNO and CTIO by Associated Universities for Research in Astronomy, all under cooperative agreements with the National 
Science Foundation. The MDM Observatory is jointly operated by the University of Michigan, Dartmouth College and the Massachusetts Institute of Technology on Kitt Peak mountain, Arizona. The Hale telescope at the PO is operated by the California Institute of Technology under a cooperative agreement with Cornell University and the Jet Propulsion Laboratory. This research was supported by NSF grants AST94-20505 to RG, AST90-14850 and AST90-23450 to MH and AST93-47714 to GW.

\section{REFERENCES}

Aaronson, M. and Mould, J. 1983, ApJ 265, 1

Aaronson, M., Bothun, G.D., Mould, J., Huchra, J., Schommer, R. and Cornell, M. 1986, ApJ 302, 536

Bahcall, N.A. and Oh, S.P. 1996, ApJ 462, L49

Bernstein, G.M., Guhathakurta, P., Raychaudhury, S., Giovanelli, R., Haynes, M.P., Herter, T. and Vogt, N.P. 1994 AJ 107, 1962

Bottinelli, L., Gouguenheim, L., Paturel, D. and Teerikorpi, P. 1986, A\&A 156, 157

Broeils, A. 1992, Ph. D. thesis, University of Groningen

Burstein, D. 1982, ApJ 253, 539

da Costa, L.N., Freudling, L.N., Wegner, G., Giovanelli, R., Haynes, M.P. and Salzer, J.J. 1996, ApJ468, L5 (paper V)

Davies, J., Phillipps, S., Boyce, D.J. and Evans, R. 1994, MNRAS 268, 984

Eisenstein, D.J. and Loeb, A.. 1996, ApJ 459, 432

Federspiel, M, Tammann, G. and Sandage, A. 1994, ApJ 430, 29

Feast, M. 1987, Observatory 107, 185

Fouqué, P., Bottinelli, L., Gouguenheim, L. and Paturel, G. 1990, ApJ 349, 1

Franx, M. and de Zeeuw, T. 1992, ApJ 392, L47.

Freudling, W.,da Costa, L.N., Wegner, G., Giovanelli, R., Haynes, M.P. and Salzer, J.J. 1995, AJ 110, 920 (Paper III)

Freudling, W.,da Costa, L.N., Wegner, G., Giovanelli, R., Haynes, M.P. and Salzer, J.J. 1995, in Potsdam Workshop, in press 
Giovanelli, R. 1996, in The Extragalactic Distance Scale, M. Livio, M. Donahue and N. Panagia, eds., Cambridge:Cambridge U. Press, in press.

Giovanelli, R., Haynes, M.P., Salzer, J.J., Wegner, G., da Costa, L.N. and Freudling, W. 1994, AJ 107, 2036 (Paper I)

Giovanelli, R., Haynes, M.P., Salzer, J.J., Wegner, G., da Costa, L.N. and Freudling, W. 1995, AJ 110, 1059 (Paper II)

Giovanelli, R., Haynes, M.P., Wegner, G., da Costa, L.N., Freudling, W. and Salzer, J.J. 1996a, ApJ 464, L99 (Paper IV)

Giovanelli, R., Haynes, M.P., Herter, T., Vogt, N.P., da Costa, L.N., Freudling, W., Salzer, J.J. and Wegner, G. 1996, AJ submitted (Paper VI)

Giovanelli, R. et al. 1996c, in preparation (Paper VIII)

Giovanelli, R., Haynes, M.P., Chamaraux, P., da Costa, L.N., Freudling, W., Salzer, J.J. and Wegner, G. 1995b, in Examining the Big Bang and Diffuse Background Radiations, proc. of IAU Symp. nr. 168, ed. by M. Kafatos and Y. Kondo, p. 183, Kluwer:Dordrecht.

Han, M. and Mould, J.R. 1992, ApJ 396, 453 (HM)

Haynes, M.P. et al. 1997, AJ in preparation

Hoffman, L. and Salpeter, E. 1996, in The Extragalactic Distance Scale, M. Livio, M. Donahue and N. Panagia, eds., Cambridge:Cambridge U. Press, in press.

Kogut, A. et al. 1993, ApJ 419, 1

Kraan-Korteweg, R.C., Cameron, L.M. and Tammann, G.A. 1988, ApJ 331, 620

Landy, S. and Szalay, A. 1992, ApJ 394, L25

Lauberts, A. 1982, The ESO/Uppsala Survey of the ESO(B) Atlas (Garching:ESO)

Lynden-Bell, D. et al. 1988, ApJ 326, 19

Malmquist, K.G. 1924, Medd. Lund Astron. Obs. Ser. II No. 32, 64

Mathewson, D.S., Ford, V.L. and Buchhorn, M. 1992, ApJS 81, 413

Nilson, P. 1973, Uppsala General Catalogue of Galaxies, Acta Univ. Upsal. Ser. V:A, Vol. 1.

Pierce, M. and Tully, R.B. 1988, ApJ 330, 579

Pierce, M. and Tully, R.B. 1992, ApJ 387, 47 
Pierce, M. and Tully, R.B. 1996, in The Extragalactic Distance Scale, M. Livio, M. Donahue and Panagia, N., eds., Cambridge:Cambridge U. Press, in press.

Rix, H.-W. and Zaritsky, D. 1995, ApJ 447, 82

Roberts, M.S. 1978, AJ 83, 1026

Sandage, A., Binggeli, B. and Tammann, G., B. 1984, AJ 90, 1759

Sandage, A., Tammann, G. and Federspiel, M. 1995, ApJ 452, 1

Sandage, A. 1994a, ApJ 430, 1

Sandage, A. 1994b, ApJ 430, 13

Schechter, P. 1980, AJ 85, 801

Silk, J. 1996, in The Universe at High z, Large Scale Structure and the Cosmic Microwave Background, E. Martinez-Gonzalez and J.-L. Sanz, eds., Springer-Verlag, in press.

Tammann, G. and Sandage, A. 1985, ApJ 294, 81

Teerikorpi, P. 1984, A\&A 141, 407

Teerikorpi, P. 1990, A\&A 234, 1

Teerikorpi, P. 1993, A\&A 280, 443

Tully, R.B. and Fisher, J.R. 1977, A\&A 54, 661

Vaucouleurs, G. de, Buta, R., Bottinelli, L., Gouguenheim, L. and Paturel, G. 1982, ApJ 254, 8

Vogt, N. P. 1995, Ph. D. thesis, Cornell University.

Willick, J.A. 1990, ApJ 351, L5

Willick, J.A. 1994, ApJS 92, 1

Willick, J.A., Courteau, S., Faber, S.M., Burstein, D., Dekel, A. 1995, ApJ 446, 12

Zwicky, F. et al. 1961-68, Catalogue of Galaxies and Clusters of Galaxies I-VI, Cal. Inst. of Tech., Pasadena. 
Fig. 1.- A graphic summary of the TF error budget. The two dotted lines represent the uncertainties arising from measurement errors and from the correction applied to observed parameters: $\epsilon_{y}$ is the error on the magnitude and $\epsilon_{x}$ is the error on the velocity widths; the latter is multiplied by the TF slope so that it can be expressed in mags. $\epsilon_{x}$ and $\epsilon_{y}$ are computed using not only the galaxies in the cluster sample (SCI), but also those in a larger field sample (SFI), providing a more accurate estimate. The solid line labelled $\epsilon_{m}$ is the total measurement error, as given by eqn. (10), and represents the total contribution of measurement and correction errors to the TF scatter. It is approximated by $\left[\left(b \epsilon_{x}\right)^{2}+\epsilon_{y}^{2}\right]^{1 / 2}$, but not exactly matched by it, because the errors $\epsilon_{x}$ and $\epsilon_{y}$ are partially correlated. The three dashed lines are the results of sums in quadrature of $\epsilon_{m}$ and an "intrinsic" scatter figure of, respectively, 0.20, 0.25 and 0.30 mag, bottom to top. The unfilled symbols are the measured, mean absolute deviation (squares) and standard deviation (circles) of the residuals from a linear TF relation. These values underestimate the scatter due to the incompleteness bias discussed in section 3.2. Finally, the solid circles and the crossed squares are respectively the incompleteness bias-corrected values of the standard deviation and mean absolute deviation: these represent the true total scatter of the TF relation. The heavy solid line is the combination in quadrature of the measurement error, $\epsilon_{m}$, and the intrinsic scatter $\epsilon_{\text {int }}$ given by eqn. (11).

Fig. 2.- Relative contributions of various components to the total measurement error $\epsilon_{m}$, for two representative cases. Refer to eqn. (10) and section 2.2 for details. Panel (a) applies to objects with fairly well determined width $\epsilon_{W} / W=0.02$ and a large value of $\gamma=1$, as it might correspond to a fairly bright and rapidly rotating galaxy. Panel (b) applies to objects with $\epsilon_{W} / W=0.07$ and a relatively small value of $\gamma=0.6$, as it might correspond to a relatively faint galaxy, with a velocity width determination of less than average quality. In both cases, the error in the determination of the magnitude (before applying extinction corrections) is assumed to be 0.05 mag.

Fig. 3. - TF diagrams of galaxies in the in + cluster samples; each cluster sample has been shifted by an amount $\Delta y$ as computed in section 6 , correcting for the cluster peculiar motion with respect to the overall sample. Different symbols are used for different types, as labelled. Panel (a) displays data uncorrected for any bias, while in panel (b) data points have been shifted by the amount indicated by eqn. (8) and Table 1 . In each panel, the solid line identifies the fit to the late spirals.

Fig. 4.- TF diagrams of galaxies of type earlier than $\mathrm{Sb}$ (panel a) and $\mathrm{Sb}$ (panel b). Data points are uncorrected for a morphological type or any other kind of bias. The dashed line in both panels is the fiducial TF relation fit to late spirals ( $\mathrm{Sbc}$ and $\mathrm{Sc}$ ), as shown in Figure 3; the solid lines identify the adopted TF relation for the two type groupings displayed here, showing the departure from the fit to later spirals. In panel (a), early type galaxies from our field galaxy sample (SFI) have been added (unfilled symbols), in order to increase the statistical significance of the adopted correction. 
Fig. 5.- Simulation of cluster incompleteness bias. A cluster sample following a UTF as indicated by the dashed straight line is generated; the individual data points are represented by unfilled circles. They are random deviates of the LF shown by the solid curve plotted along the magnitude axis, and extracted to have completeness characteristics as shown by the shaded magnitude histogram. The completeness function of the cluster is the ratio of the histogram to the LF. A running average of the magnitudes of objects in the sample, contained within non-overlapping bins of velocity width, is indicated by the solid symbols connected by the heavy solid line: as the sample becomes progressively more incomplete towards smaller velocity widths, the mean magnitude increasingly departs from the UTF. Note that the scatter of the data has been exaggerated to an amplitude twice as large as that indicated by eqn. (3).

Fig. 6.- Bias correction for the Coma cluster. (a) unfilled symbols represent the raw data for galaxies in the in+ sample of the cluster. After the application of the morphological type correction discussed in section 3.2, the points shift to the locations of the filled circles. The dashed straight line identifies a direct fit to the morphological type-corrected data. The luminosity function of the galaxy population from which the sample is assumed to be extracted is shown as a solid line plotted along the vertical axis, together with the shaded histogram of magnitudes for the sample. The completeness function of the sample is the ratio between the histogram and the LF. (b) Amplitude of the bias associated with the incompleteness of the sample, as described in section 3.2. This was computed by producing $n_{\text {times }}=800$ independent simulations of the Coma sample. (c) Effect of the incompleteness bias on the TF scatter: the ratio $\epsilon_{\text {meas }} / \epsilon_{\text {true }}$ is between the mean measured scatter in $n_{\text {times }}=800$ independent simulations of the Coma sample and the actual value of the scatter input with the model. The measured scatter between $\log W=2.3$ and 2.5 is underestimated by about $10 \%$. (d) Coma sample data after the bias correction shown in panel (b) has been applied, point by point. The dashed line is the same as that in panel (a); it has been added to aid eye estimate of the amplitude of the incompleteness bias correction.

Fig. 7.- Dependence of the incompleteness bias correction for the Coma in+ sample on the amplitude of the scatter. Analogous plots to those in Figure $6 \mathrm{~b}$ and $6 \mathrm{c}$ have been produced, assuming different amplitudes of the TF scatter. The solid lines were computed for a TF scatter amplitude as estimated in section 2 and parametrized by eqn. (7); they are the same as shown in Figure 6. The long-dash and the dotted lines assume scatter amplitudes respectively 1.5 and 2 times larger than that given by eqn. (3). 
Fig. 8.- Dependence of the incompleteness bias correction for the Coma in+ sample on the shape of the LF. Analogous plots to those in Figure 6b,c and Figure 7 have been produced, assuming different shapes for the LF of the population from which the sample is supposed to be extracted: the two funtions are shown as an inset in panel (b); they differ by the value of the exponent $\alpha$ of the power law part of the Schechter representation of the LF, the solid line refers to $\alpha=-0.50$ and the dotted one to $\alpha-0.85$. Correspondingly, the incompleteness bias is shown for the two cases as solid (filled symbols) and dotted (unfilled symbols) lines.

Fig. 9.- Dependence of the incompleteness bias correction for the Coma in+ sample on the slope of the TF fit. Analogous plots to those in Figure 6b,c, Figure 7 and Figure 8 have been produced, assuming slopes of respectively -7.0 (solid line, filled symbols) and -8.0 (dotted line, unfilled symbols) for the TF relation.

Fig. 10.- Completeness histograms for each of the in+ cluster samples. The shaded histograms are referred as the function $h^{\prime}$ in section 3.2, while the dashed lines are the fitted completeness functions $c(y)$. Parameters of the $c(y)$ functions are listed in Table 2.

Fig. 11.- Incompleteness bias, in magnitudes, computed for each in+ cluster sample. Refer to section 3.2 for details.

Fig. 12.- Incompleteness bias correction applied to individual galaxies in the in + cluster samples, plotted versus apparent magnitude. In panel (a), the bias correction is computed for a value of $\alpha=-0.50$ for the power law exponent of the faint end of the LF, while in panel (b) a value of $\alpha=-0.85$ is used.

Fig. 13.- The relative bias $B$, measured in units of the mean TF scatter, is plotted versus the parameter $A$, which is a measure of the closeness to the edge of the magnitude limit of the catalog from which sample objects are extracted. The "catalog edge" occurs at $A=0$. The values of this bias were estimated for a strictly apparent magnitude limited sample using Willick's (1994) approach (solid line), and our Monte Carlo approach (unfilled squares). Both results agree well with each other. The solid symbols display the bias expected for a sample with completeness characteristics similar to those of our in + global sample, estimated using a Monte Carlo approach. See section 3.3 for details.

Fig. 14.- Illustration of the cluster size-incompleteness bias. The curve labelled $c(y)$ describes the completeness function of the sample, which is complete on the left side of the plot. The curve labelled $E(y)$ describes the distribution of magnitudes $y$ to be expected from a population of galaxies of fixed magnitude $y_{\circ}$, spread over the cluster; the spread reflects the depth of the cluster along the line of sight. The heavy tracing is the product $c(y) E(y)$, and the offset between the centroid of $c E$ and $y_{\circ}$ is the bias. 
Fig. 15. - Estimates of the homogeneous MBC. The solid line is the MBC for a single object for which the distance modulus uncertainty is given by eqn. (3). By comparison, dashed lines display the MBC for constant uncertainties on the distance modulus, as posted in mag.

Fig. 16. - TF magnitude residuals plotted versus projected radial distance from cluster centers. In panel (a), all galaxies in the in+ samples are included. In panel (b), only galaxies in the 12 higher density clusters (A262, A400, Cancer, Hydra, A1367, Cen30, Coma, ESO508, A3574, A2197/9, A2634 and A2666) are plotted; in panel (c) galaxies in the remaining 11 clusters are in display.

Fig. 17. - TF relations for individual cluster. Filled symbols refer to galaxies in in samples; additional objects in in+ samples are plotted as unfilled symbols. The data are uncorrected for any of the biases discussed in section 3. The unfilled symbols for A2197 include peripheral cluster members as well as galaxies in A2199. The same fiducial line of slope -7.60 has been drawn in every graph. Absolute magnitudes are computed assuming the clusters are at rest with respect to the CMB reference frame, i.e. no correction for cluster motion is applied.

Fig. 18. - TF relations for individual clusters. All galaxies in the in + samples are plotted; uncertainties $\epsilon_{x}$ and $\epsilon_{y}$, arising from both measurement errors and from corrections such as internal extinction, turbulence and disk inclination, are plotted at each data point. No allowance for intrinsic scatter is made in the error bars. The location of the data points is that obtained after bias corrections discussed in section 3 have been applied. The same fiducial line of slope -7.60 has been drawn in every graph. Absolute magnitudes are computed assuming the clusters are at rest with respect to the $\mathrm{CMB}$ reference frame, i.e. no correction for cluster motion is applied. Data for the two clusters A2197 and A2199 have been plotted in the same graph. For comparison, along the $\mathrm{y}$-axis of the panels on the left we show the assumed LF of the population from which each sample is estimated to be an incomplete representation.

Fig. 19.- Distribution of magnitude residuals for each in cluster sample, about a TF relation of slope -7.62 and where the mean residual for each cluster is forced to be zero, plotted versus the log of each galaxy's radial velocity, minus the log of the cluster systemic radial velocity. A dashed line of slope 5 is drawn in each graph, indicating the locus about which galaxies that are not cluster members should scatter.

Fig. 20.- Distribution of magnitude residuals for all galaxies in in cluster samples displayed in Figure 19. A dashed line of slope 5 is drawn in each graph, indicating the locus about which galaxies that are not cluster members should scatter. There is no significant indication of sample contamination by foreground or background objects. 
Fig. 21. - Global set, obtained by combining the cluster samples as described in section 6.1. Direct and bivariate regression fits are inset. In panel (a), the result of the combination of in samples is shown; in panel (b), the analogous plot for in+ samples is displayed, while in panel (c) we show the same data points as in panel (b), with their associated measurement error bars.

Fig. 22. - Illustration of the underestimation of the TF scatter due to the incompleteness bias for each in+ cluster sample, plotted as a function of velocity width, for the the case of the global set combination shown in Figure 21(b). The smooth line superimposed to the data points represents a parametrization of the scatter underestimation, which was applied in the derivation of the position of the filled symbols in Figure 1.

Fig. 23. - (a) Magnitude residuals with respect to the bivariate TF relation for the in + global set, plotted versus isophotal angular diameter, measured at the $23.5 \mathrm{mag} \operatorname{arcsec}^{-2}$ isophote; (b) same residuals, plotted versus apparent magnitude. In both cases, large solid symbols refer to running averages.

Fig. 24. - (a) Magnitude residuals with respect to the bivariate TF relation for the in + global set, plotted versus galaxy redshift; (b) same residuals, plotted versus disk inclination. In both cases, large solid symbols refer to runnign averages. 
TABLE 2

Fit Coefficients. Individual Cluster Samples.

\begin{tabular}{|c|c|c|c|c|c|c|c|c|c|c|c|}
\hline $\begin{array}{c}\text { Sample } \\
(1)\end{array}$ & $\begin{array}{l}\mathrm{N} \\
(2)\end{array}$ & $\begin{array}{c}a_{d i r} \\
(3)\end{array}$ & $\begin{array}{c}b_{d i r} \\
(4)\end{array}$ & $\begin{array}{c}a_{i n v} \\
(5)\end{array}$ & $\begin{array}{c}b_{i n v} \\
(6)\end{array}$ & $\begin{array}{l}a_{b i} \\
(7)\end{array}$ & $\begin{array}{l}b_{b i} \\
(8)\end{array}$ & $\begin{array}{c}\epsilon_{a} \\
(9)\end{array}$ & $\begin{array}{c}\epsilon_{b} \\
(10)\end{array}$ & $\begin{array}{l}\sigma_{b i} \\
(11)\end{array}$ & $\begin{array}{l}\sigma_{a b s} \\
(12)\end{array}$ \\
\hline N383 in & 8 & -21.00 & -5.25 & -21.10 & -6.14 & -21.02 & -5.62 & 0.13 & 1.00 & 0.30 & 0.23 \\
\hline N383 in + & 21 & -20.99 & -6.85 & -21.11 & -7.73 & -21.04 & -7.14 & 0.08 & 0.54 & 0.35 & 0.32 \\
\hline N507 in & 10 & -21.11 & -8.11 & -21.14 & -7.84 & -21.11 & -8.08 & 0.10 & 0.56 & 0.34 & 0.25 \\
\hline N507 in + & 14 & -21.04 & -8.11 & -21.08 & -8.23 & -21.07 & -7.93 & 0.09 & 0.56 & 0.35 & 0.25 \\
\hline A262 in & 17 & -21.05 & -6.80 & -21.00 & -7.20 & -20.97 & -7.10 & 0.08 & 0.56 & 0.29 & 0.27 \\
\hline A262 in + & 31 & -21.08 & -7.32 & -21.11 & -7.54 & -21.05 & -7.34 & 0.06 & 0.42 & 0.32 & 0.28 \\
\hline A400 in & 10 & -20.94 & -7.47 & -20.93 & -8.38 & -20.91 & -7.97 & 0.13 & 1.19 & 0.22 & 0.17 \\
\hline A400 in + & 25 & -20.98 & -7.47 & -21.02 & -7.95 & -20.97 & -7.72 & 0.07 & 0.74 & 0.22 & 0.17 \\
\hline Eridanus in & 25 & -20.59 & -7.88 & -20.84 & -9.23 & -20.69 & -8.31 & 0.12 & 0.56 & 0.51 & 0.36 \\
\hline Eridanus in+ & 34 & -20.62 & -7.80 & -20.82 & -8.94 & -20.71 & -8.20 & 0.09 & 0.46 & 0.45 & 0.35 \\
\hline Fornax in & 26 & -20.85 & -7.11 & -20.92 & -7.85 & -20.88 & -7.70 & 0.09 & 0.35 & 0.45 & 0.45 \\
\hline Fornax in+ & 39 & -20.72 & -7.08 & -20.84 & -8.07 & -20.77 & -7.69 & 0.07 & 0.32 & 0.49 & 0.43 \\
\hline Cancer in & 17 & -21.22 & -8.97 & -21.29 & -9.26 & -21.22 & -8.96 & 0.11 & 0.72 & 0.32 & 0.24 \\
\hline Cancer in+ & 26 & -21.04 & -7.78 & -21.13 & -8.64 & -21.03 & -7.83 & 0.08 & 0.56 & 0.35 & 0.26 \\
\hline Antlia in & 14 & -21.10 & -9.08 & -21.13 & -9.58 & -21.13 & -9.00 & 0.11 & 1.03 & 0.25 & 0.18 \\
\hline Antlia in+ & 27 & -21.15 & -8.46 & -21.23 & -8.66 & -21.21 & -8.07 & 0.08 & 0.56 & 0.36 & 0.27 \\
\hline Hydra in & 18 & -20.78 & -6.92 & -20.86 & -7.51 & -20.80 & -7.14 & 0.08 & 0.74 & 0.30 & 0.25 \\
\hline Hydra in + & 25 & -20.87 & -7.00 & -20.93 & -7.89 & -20.88 & -7.26 & 0.07 & 0.71 & 0.30 & 0.24 \\
\hline N3557 in & 7 & -21.06 & -6.10 & -21.13 & -6.45 & -21 & -6.01 & 0.14 & 1.38 & 0.21 & 0.17 \\
\hline N3557 in+ & 11 & -21.16 & -7.31 & -21.23 & -7.39 & -21 & -7.25 & 0.12 & 0.95 & 0.31 & 0.24 \\
\hline A1367 in & 33 & -21.04 & -7.55 & -20.98 & -8.62 & -21 & -7.94 & 0.07 & 0.65 & 0.31 & 0.24 \\
\hline A1367 in+ & 35 & -21.04 & -7.51 & -20.98 & -8.62 & -21 & -7.90 & 0.07 & 0.65 & 0.31 & 0.23 \\
\hline Ursa Major in & 29 & -20.45 & -9.04 & -20.50 & -9.42 & -20 & -9.17 & 0.08 & 0.44 & 0.30 & 0.23 \\
\hline Ursa Major in+ & 30 & -20.45 & -9.08 & -20.50 & -9.59 & -20 & -9.27 & 0.08 & 0.44 & 0.32 & 0.24 \\
\hline Cen30 in & 23 & -21.11 & -7.42 & -21.25 & -8.66 & -21 & -7.97 & 0.10 & 0.69 & 0.40 & 0.33 \\
\hline Cen30 in+ & 38 & -21.23 & -7.66 & -21.29 & -8.64 & -21 & -8.14 & 0.07 & 0.50 & 0.38 & 0.32 \\
\hline A1656 in & 29 & -21.11 & -6.77 & -21.17 & -6.74 & -21 & -6.81 & 0.07 & 0.55 & 0.23 & 0.17 \\
\hline A1656 in+ & 41 & -21.12 & -6.72 & -21.16 & -7.17 & -21 & -6.79 & 0.06 & 0.48 & 0.27 & 0.19 \\
\hline ESO508 in & 8 & -21.34 & -7.08 & -21.48 & -7.19 & -21 & -7.30 & 0.19 & 1.22 & 0.24 & 0.19 \\
\hline ESO508 in+ & 17 & -21.26 & -6.86 & -21.38 & -7.37 & -21 & -6.97 & 0.11 & 0.87 & 0.25 & 0.18 \\
\hline A3574 in & 9 & -21.05 & -6.29 & -21.05 & -6.80 & -21 & -6.47 & 0.17 & 1.52 & 0.17 & 0.13 \\
\hline A3574 in+ & 20 & -21.02 & -6.92 & -21.08 & -6.99 & -21 & -7.09 & 0.08 & 0.69 & 0.17 & 0.14 \\
\hline A2197 in & 11 & -20.93 & -7.99 & -20.69 & -13.28 & -20 & -9.19 & 0.19 & 2.02 & 0.40 & 0.28 \\
\hline A2199 in & 9 & -21.17 & -6.07 & -21.32 & -5.49 & -21 & -6.20 & 0.23 & 1.20 & 0.15 & 0.12 \\
\hline A2197/9 in+ & 25 & -21.02 & -7.03 & -21.04 & -8.02 & -2 & -7.43 & 0.12 & 0.83 & 0.30 & 0.22 \\
\hline Pavo 2 in & 6 & & -8.11 & & -7.58 & -21 & .08 & 0.18 & 1.17 & 0.19 & 0.18 \\
\hline Pavo 2 in + & 18 & -21.14 & -6.63 & -21.19 & -7.06 & -21.13 & -6.75 & 0.09 & 0.65 & 0.35 & 0.28 \\
\hline Pavo in & 5 & -21.17 & -9.15 & -21.69 & -20.87 & -21.31 & -11.94 & 0.35 & 6.10 & 0.33 & 0.22 \\
\hline Pavo in + & 10 & -21.05 & -7.31 & -21.10 & -8.77 & -21.00 & -7.58 & 0.13 & 1.30 & 0.37 & 0.33 \\
\hline MDL59 in & 10 & -20.54 & -7.82 & -20.62 & -8.17 & -20.58 & -8.02 & 0.19 & 1.04 & 0.31 & 0.25 \\
\hline MDL59 in+ & 23 & -20.55 & -7.40 & -20.62 & -8.16 & -20.57 & -7.71 & 0.09 & 0.51 & 0.38 & 0.32 \\
\hline Pegasus in & 12 & -20.74 & -5.99 & -20.85 & -6.57 & -20.77 & -6.31 & 0.15 & 0.84 & 0.26 & 0.22 \\
\hline Pegasus in + & 17 & -20.66 & -6.11 & -20.87 & -7.41 & -20.70 & -6.60 & 0.13 & 0.74 & 0.34 & 0.27 \\
\hline A2634 in & 15 & -21.00 & -7.28 & -21.09 & -7.31 & -20.97 & -7.50 & 0.10 & 0.70 & 0.27 & 0.21 \\
\hline A2634 in + & 19 & -20.99 & -7.00 & -21.08 & -6.99 & -20.98 & -7.14 & 0.09 & 0.61 & 0.26 & 0.20 \\
\hline A2666 in & 9 & -21.04 & -6.58 & -21.04 & -7.18 & -21.01 & -6.89 & 0.14 & 1.24 & 0.21 & 0.18 \\
\hline
\end{tabular}



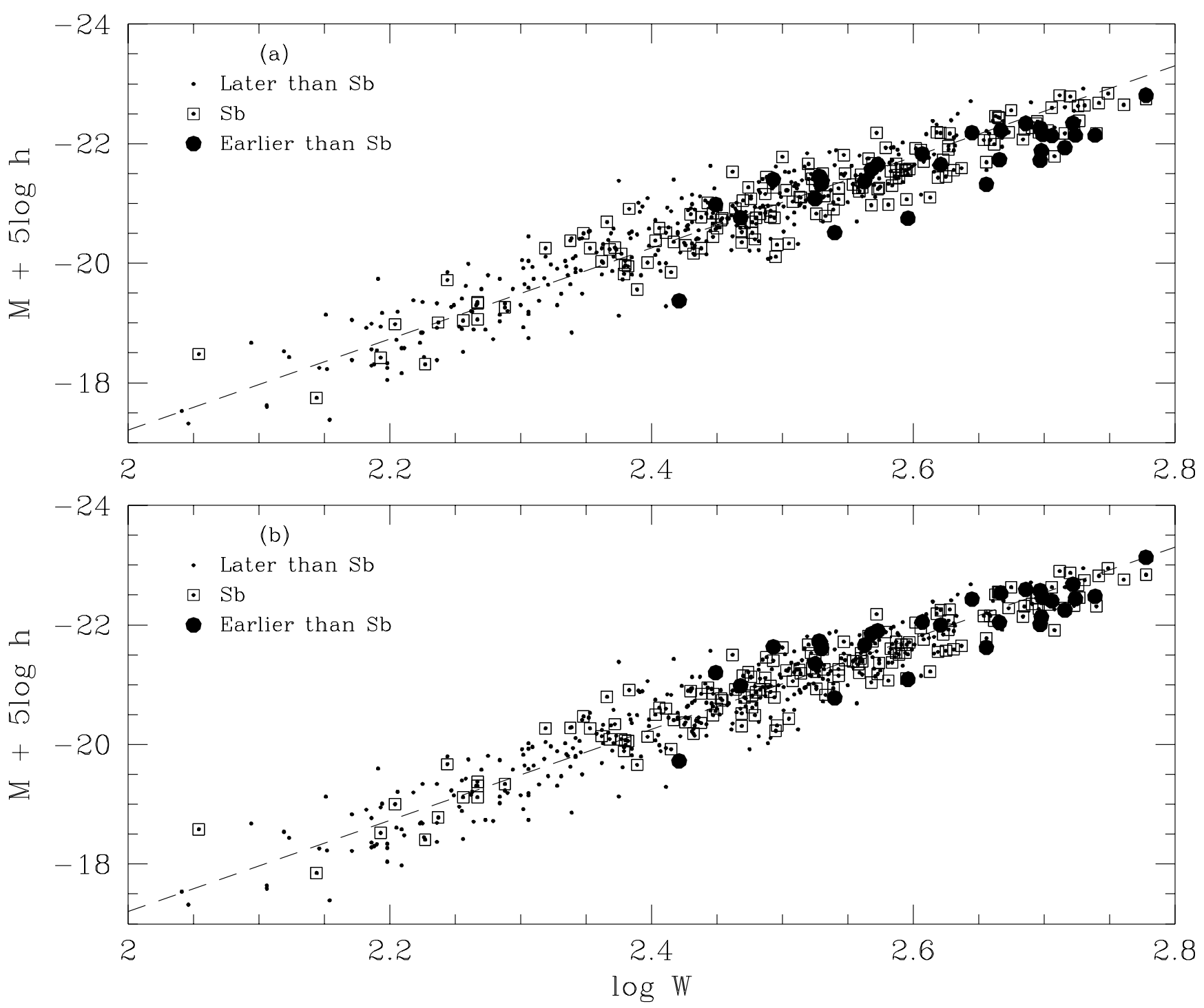
TABLE 3

Fit Offsets. Individual Cluster Samples.

\begin{tabular}{|c|c|c|c|c|c|c|}
\hline $\begin{array}{c}\text { Sample } \\
(1)\end{array}$ & $\begin{array}{l}\mathrm{N} \\
(2)\end{array}$ & $\begin{array}{c}\Delta y_{\circ} \dagger \\
(3)\end{array}$ & $\begin{array}{c}\Delta y_{1} \dagger \\
(4)\end{array}$ & $\begin{array}{c}\Delta y_{2} \dagger \\
(5)\end{array}$ & $\begin{array}{r}\Delta y_{3} \\
(6)\end{array}$ & $\begin{array}{c}\dagger \Delta y_{4} \dagger \\
(7)\end{array}$ \\
\hline N383 in & 8 & -0.02 & $+0.02(13)$ & -0.01 & -0.03 & -0.08 \\
\hline N383 in + & 21 & +0.04 & $+0.01(08)$ & +0.01 & +0.01 & -0.01 \\
\hline N507 in & 10 & -0.02 & $-0.13(11)$ & -0.14 & -0.08 & -0.10 \\
\hline N507 in + & 14 & +0.02 & $-0.05(09)$ & -0.06 & -0.02 & -0.04 \\
\hline A262 in & 17 & +0.02 & $-0.01(08)$ & +0.03 & +0.01 & +0.04 \\
\hline A262 in + & 31 & -0.04 & $-0.06(06)$ & -0.02 & -0.04 & -0.01 \\
\hline A400 in & 10 & +0.09 & $+0.09(13)$ & +0.06 & +0.10 & +0.06 \\
\hline A400 in + & 25 & +0.05 & $+0.04(07)$ & +0.02 & +0.06 & +0.04 \\
\hline Eridanus in & 25 & +0.49 & $+0.43(12)$ & +0.42 & +0.48 & +0.41 \\
\hline Eridanus in + & 34 & +0.46 & $+0.39(09)$ & +0.38 & +0.43 & +0.38 \\
\hline Fornax in & 26 & +0.13 & $+0.06(10)$ & +0.06 & +0.09 & +0.01 \\
\hline Fornax in + & 39 & +0.23 & $+0.19(09)$ & +0.16 & +0.21 & +0.14 \\
\hline Cancer in & 17 & -0.04 & $-0.12(11)$ & -0.15 & -0.06 & -0.11 \\
\hline Cancer in + & 26 & -0.04 & $-0.01(08)$ & -0.05 & -0.00 & -0.05 \\
\hline Antlia in & 14 & -0.13 & $-0.16(11)$ & -0.15 & -0.09 & -0.09 \\
\hline Antlia in + & 27 & -0.10 & $-0.16(08)$ & -0.15 & -0.12 & -0.10 \\
\hline Hydra in & 18 & +0.21 & $+0.23(08)$ & +0.24 & +0.24 & +0.25 \\
\hline Hydra in + & 25 & +0.14 & $+0.15(07)$ & +0.15 & +0.18 & +0.18 \\
\hline N3557 in & 7 & -0.09 & $-0.08(14)$ & -0.06 & -0.03 & -0.02 \\
\hline N3557 in + & 11 & -0.20 & $-0.17(11)$ & -0.14 & -0.12 & -0.09 \\
\hline A1367 in & 33 & -0.03 & $-0.04(07)$ & -0.04 & +0.02 & +0.01 \\
\hline A1367 in + & 35 & -0.02 & $-0.03(06)$ & -0.04 & +0.00 & -0.00 \\
\hline Ursa Major in & 29 & +0.70 & $+0.65(08)$ & +0.65 & +0.75 & +0.70 \\
\hline Ursa Major in+ & 30 & +0.76 & $+0.68(08)$ & +0.67 & +0.77 & +0.73 \\
\hline Cen30 in & 23 & -0.12 & $-0.14(10)$ & -0.17 & -0.09 & -0.15 \\
\hline Cen30 in + & 38 & -0.20 & $-0.23(07)$ & -0.23 & -0.18 & -0.21 \\
\hline A1656 in & 29 & -0.06 & $-0.05(07)$ & -0.07 & -0.03 & -0.06 \\
\hline A1656 in + & 41 & -0.05 & $-0.06(06)$ & -0.08 & -0.05 & -0.07 \\
\hline ESO508 in & 8 & -0.43 & $-0.39(19)$ & -0.38 & -0.36 & -0.40 \\
\hline ESO508 in+ & 17 & -0.32 & $-0.31(10)$ & -0.31 & -0.28 & -0.31 \\
\hline A3574 in & 9 & +0.07 & $+0.08(17)$ & +0.06 & +0.09 & +0.07 \\
\hline A 3574 in + & 20 & -0.03 & $+0.00(08)$ & +0.01 & +0.02 & +0.02 \\
\hline A2197 in & 11 & -0.04 & $+0.05(19)$ & +0.03 & +0.10 & +0.09 \\
\hline A2199 in & 9 & +0.06 & $+0.10(23)$ & +0.07 & +0.03 & +0.03 \\
\hline A2197/9 in + & 25 & +0.01 & $+0.06(10)$ & +0.05 & +0.04 & +0.05 \\
\hline Pavo 2 in & 6 & -0.17 & $-0.15(18)$ & -0.14 & -0.10 & -0.08 \\
\hline Pavo 2 in + & 18 & -0.10 & $-0.07(08)$ & -0.05 & -0.09 & -0.06 \\
\hline Pavo in & 5 & -0.08 & $-0.13(23)$ & -0.15 & -0.05 & -0.10 \\
\hline Pavo in + & 10 & -0.06 & $-0.07(12)$ & -0.07 & -0.00 & -0.03 \\
\hline MDL59 in & 10 & +0.49 & $+0.48(18)$ & +0.47 & +0.53 & +0.48 \\
\hline MDL59 in + & 23 & +0.46 & $+0.43(09)$ & +0.41 & +0.46 & +0.41 \\
\hline Pegasus in & 12 & +0.05 & $+0.05(13)$ & +0.03 & +0.06 & -0.01 \\
\hline Pegasus in + & 17 & +0.14 & $+0.14(11)$ & +0.11 & +0.14 & +0.07 \\
\hline A2634 in & 15 & -0.03 & $+0.03(09)$ & +0.04 & +0.04 & +0.11 \\
\hline A2634 in+ & 19 & +0.01 & $+0.07(08)$ & +0.08 & +0.05 & +0.07 \\
\hline A2666 in & 9 & +0.04 & $+0.05(13)$ & +0.04 & +0.05 & +0.04 \\
\hline
\end{tabular}

${ }^{\dagger} \mathrm{Col}(3)$ : linear TF; offset computed with no incompleteness correction.

${ }^{\dagger} \mathrm{Col}(4)$ : linear TF; incompleteness bias for $\alpha_{L F}=-0.50$.

${ }^{\dagger} \mathrm{Col}$ (5): linear TF; incompleteness bias for $\alpha_{L F}=-0.85$.

${ }^{\dagger} \mathrm{Col}$ (6): quadr. TF; incompleteness bias for $\alpha_{L F}=-0.50$.

${ }^{\dagger} \mathrm{Col}(7)$ : quadr. TF; incompleteness bias for $\alpha_{L F}=-0.85$. 

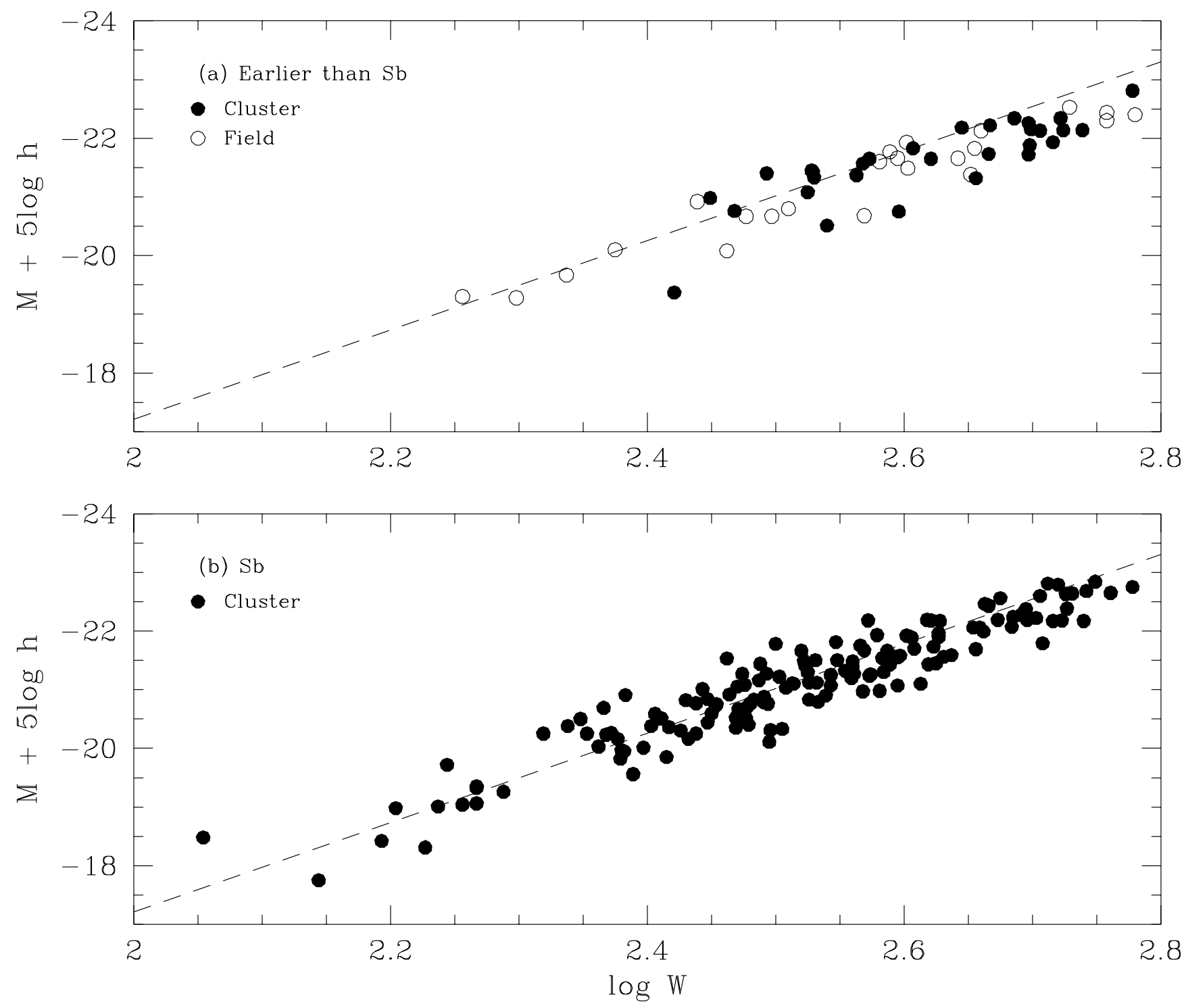
TABLE 4

Linear Fit Coefficients. Global Sets

\begin{tabular}{|c|c|c|c|c|c|c|c|c|c|c|c|c|}
\hline $\begin{array}{c}\text { Sample } \\
(1)\end{array}$ & $\begin{array}{l}\mathrm{N} \\
(2)\end{array}$ & $\begin{array}{c}a_{d i r} \\
(3)\end{array}$ & $\begin{array}{c}b_{d i r} \\
(4)\end{array}$ & $\begin{array}{c}a_{i n v} \\
(5)\end{array}$ & $\begin{array}{c}b_{i n v} \\
(6)\end{array}$ & $\begin{array}{l}a_{b i} \\
(7)\end{array}$ & $\begin{array}{l}b_{b i} \\
(8)\end{array}$ & $\begin{array}{c}\epsilon_{a} \\
(9)\end{array}$ & $\begin{array}{c}\epsilon_{b} \\
(10)\end{array}$ & $\begin{array}{l}\sigma_{b i} \\
(11)\end{array}$ & $\begin{array}{l}\sigma_{a b s} \\
(12)\end{array}$ & $\begin{array}{r}b_{p} \\
(13)\end{array}$ \\
\hline in no incompl. bias & 360 & -21.09 & -7.16 & -21.10 & -7.94 & -21.09 & -7.42 & 0.02 & 0.13 & 0.35 & 0.29 & -7.42 \\
\hline in + no incompl. bias & 555 & -21.09 & -7.12 & -21.10 & -7.95 & -21.10 & -7.32 & 0.02 & 0.10 & 0.36 & 0.29 & -7.34 \\
\hline \multicolumn{13}{|l|}{$\alpha_{L F}=-0.50$} \\
\hline in & 360 & -21.00 & -7.52 & & & -21.00 & -7.76 & 0.02 & 0.13 & 0.34 & 0.28 & -7.75 \\
\hline in $2.5 \sigma$ clip & 350 & -21.00 & -7.60 & & & -21.00 & -7.67 & 0.02 & 0.13 & 0.31 & 0.25 & -7.70 \\
\hline in $60^{\circ}<i<80^{\circ}$ & 187 & -21.00 & -7.47 & & & -21.00 & -7.68 & 0.03 & 0.17 & 0.31 & 0.26 & -7.66 \\
\hline int & 555 & -21.01 & -7.48 & & & -21.01 & -7.68 & 0.02 & 0.10 & 0.34 & 0.28 & -7.67 \\
\hline in $+i>45^{\circ}$ & 534 & -21.01 & -7.45 & & & -21.01 & -7.66 & 0.02 & 0.11 & 0.34 & 0.27 & -7.66 \\
\hline in $+W>150, y<-18.5$ & 514 & -21.02 & -7.33 & & & -21.02 & -7.50 & 0.02 & 0.13 & 0.33 & 0.26 & -7.50 \\
\hline in $+60^{\circ}<i<80^{\circ}$ & 281 & -21.02 & -7.41 & & & -21.03 & -7.60 & 0.02 & 0.14 & 0.32 & 0.27 & -7.62 \\
\hline in $+2.5 \sigma$ clip & 541 & -21.01 & -7.50 & & & -21.01 & -7.59 & 0.02 & 0.11 & 0.32 & 0.26 & -7.60 \\
\hline in $+T \leq S b$ & 199 & -21.02 & -7.17 & & & -21.01 & -7.50 & 0.03 & 0.19 & 0.32 & 0.26 & -7.45 \\
\hline in $+T>S b$ & 356 & -21.02 & -7.60 & & & -21.03 & -7.83 & 0.02 & 0.14 & 0.36 & 0.29 & -7.84 \\
\hline \multicolumn{13}{|l|}{$\alpha_{L F}=-0.85$} \\
\hline in & 360 & -20.96 & -7.67 & & & -20.96 & -7.93 & 0.02 & 0.13 & 0.34 & 0.27 & -7.96 \\
\hline in $2.5 \sigma$ clip & 348 & -20.97 & -7.65 & & & -20.97 & -7.79 & 0.02 & 0.13 & 0.30 & 0.25 & -7.83 \\
\hline in $60^{\circ}<i<80^{\circ}$ & 187 & -20.96 & -7.60 & & & -20.96 & -7.84 & 0.03 & 0.18 & 0.31 & 0.26 & -7.81 \\
\hline in + & 555 & -20.98 & -7.62 & & & -20.97 & -7.84 & 0.02 & 0.10 & 0.34 & 0.28 & -7.84 \\
\hline in $+i>45^{\circ}$ & 534 & -20.98 & -7.59 & & & -20.98 & -7.82 & 0.02 & 0.11 & 0.34 & 0.27 & -7.83 \\
\hline in $+W>150, y<-18.5$ & 514 & -20.98 & -7.50 & & & -20.98 & -7.67 & 0.02 & 0.12 & 0.33 & 0.26 & -7.65 \\
\hline in $+60^{\circ}<i<80^{\circ}$ & 281 & -20.99 & -7.53 & & & -20.99 & -7.75 & 0.02 & 0.14 & 0.33 & 0.27 & -7.77 \\
\hline in $+2.5 \sigma$ clip & 539 & -20.98 & -7.60 & & & -20.98 & -7.70 & 0.02 & 0.11 & 0.33 & 0.26 & -7.72 \\
\hline in $+T \leq S b$ & 199 & -20.98 & -7.36 & & & -20.96 & -7.68 & 0.03 & 0.19 & 0.31 & 0.25 & -7.66 \\
\hline in $+T>S b$ & 356 & -20.98 & -7.72 & & & -21.00 & -8.00 & 0.02 & 0.14 & 0.36 & 0.29 & -7.99 \\
\hline
\end{tabular}




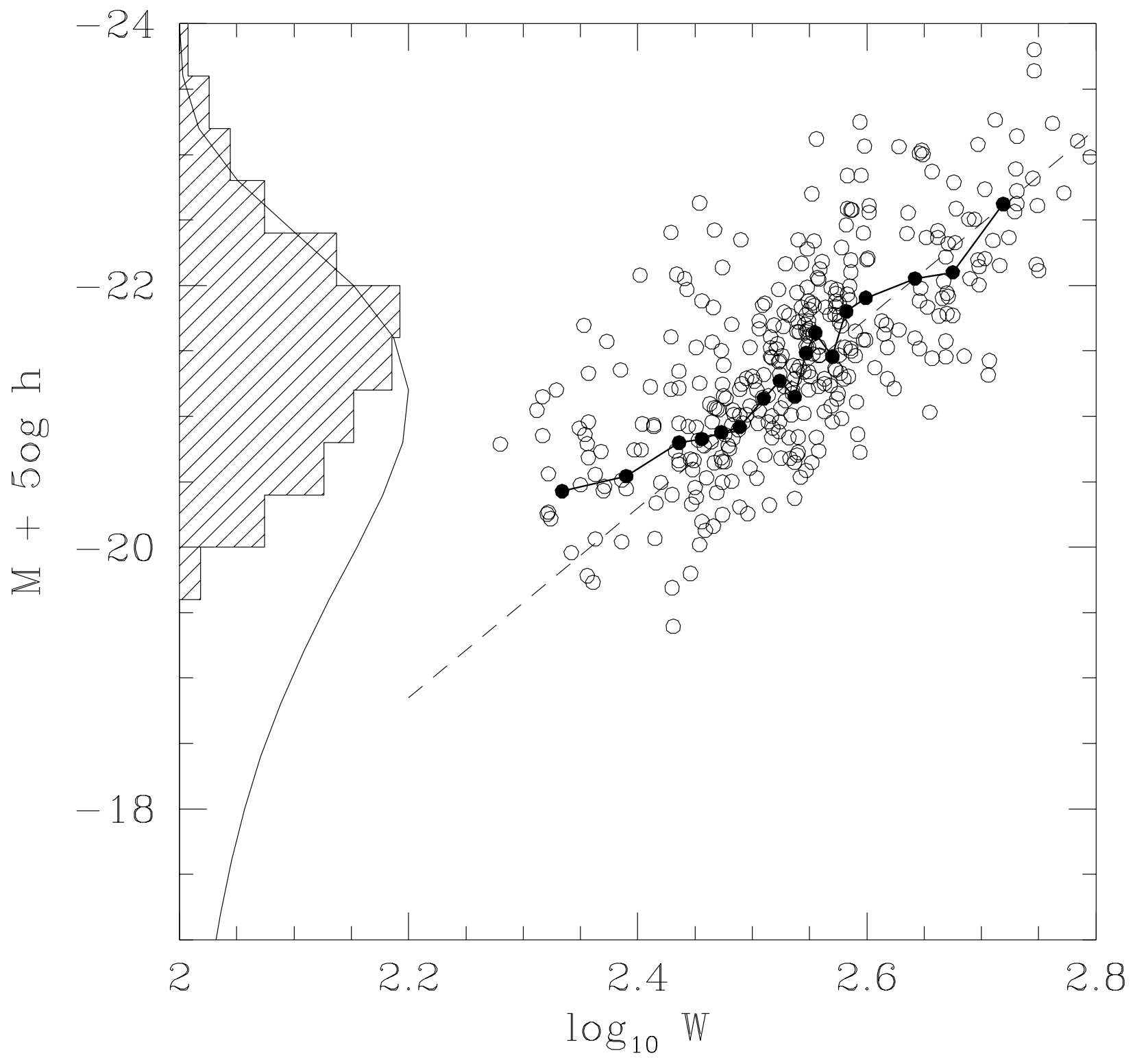

fig. 5 
TABLE 5

Quadratic Fit Coefficients. Global Sets

\begin{tabular}{|c|c|c|c|c|c|}
\hline $\begin{array}{c}\text { Sample } \\
\text { (1) }\end{array}$ & $\begin{array}{l}\mathrm{N} \\
(2)\end{array}$ & $\begin{array}{l}c_{\circ} \\
(3)\end{array}$ & $\begin{array}{l}c_{1} \\
(4)\end{array}$ & $\begin{array}{l}c_{2} \\
(5)\end{array}$ & $\begin{array}{c}\sigma \\
(6)\end{array}$ \\
\hline in (no incompl. bias) & 360 & -21.03 & -6.84 & 1.93 & 0.33 \\
\hline in + (no incompl. bias) & 555 & -21.05 & -6.76 & 2.22 & 0.34 \\
\hline$\alpha_{L F}=-0.50$ & & & & & \\
\hline in & 360 & -21.01 & -7.42 & 1.80 & 0.33 \\
\hline in + & 555 & -21.06 & -7.31 & 1.42 & 0.33 \\
\hline$\alpha_{L F}=-0.85$ & & & & & \\
\hline in & 360 & -21.01 & -7.66 & 1.06 & 0.33 \\
\hline in + & 555 & -21.01 & -7.53 & 1.35 & 0.33 \\
\hline
\end{tabular}



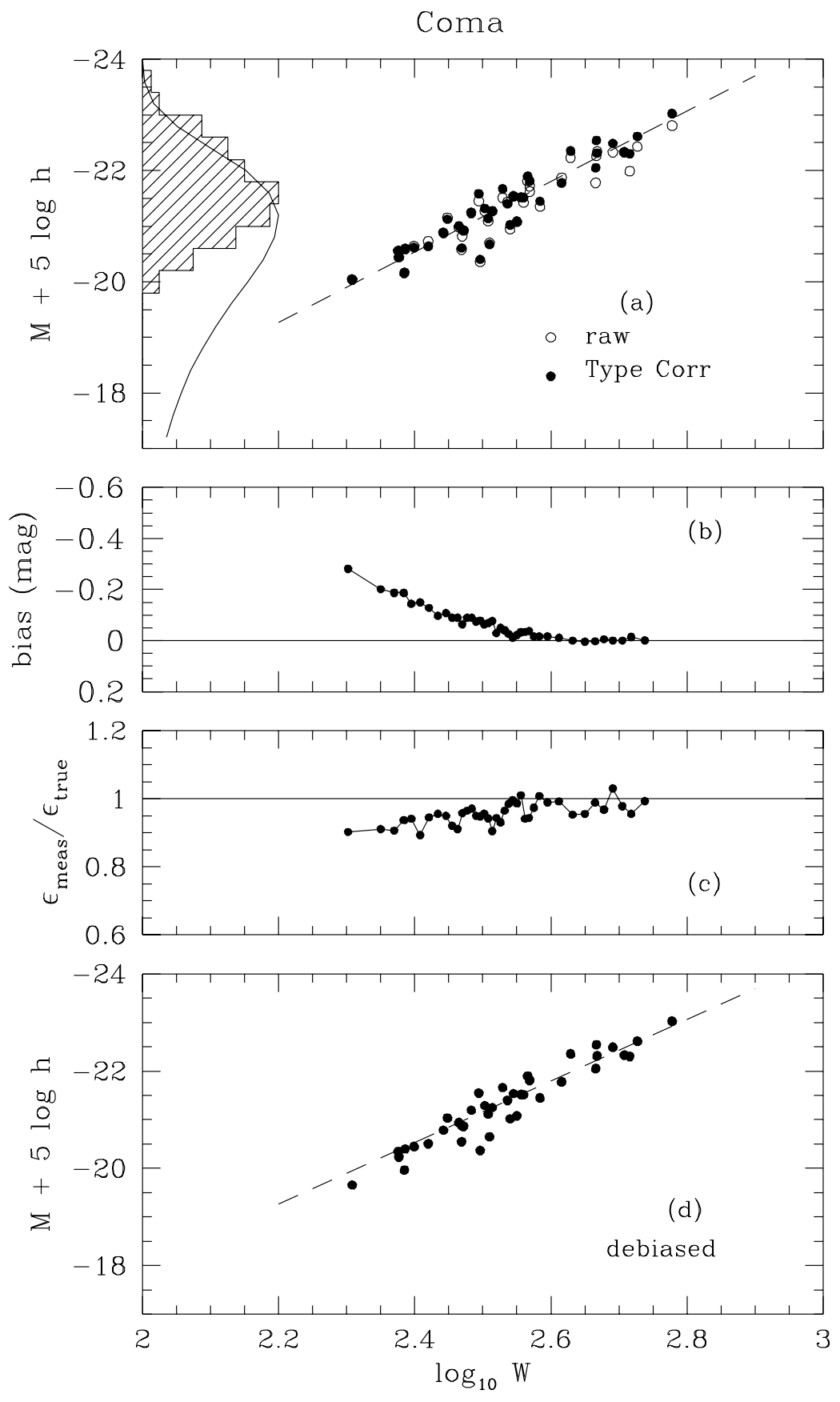


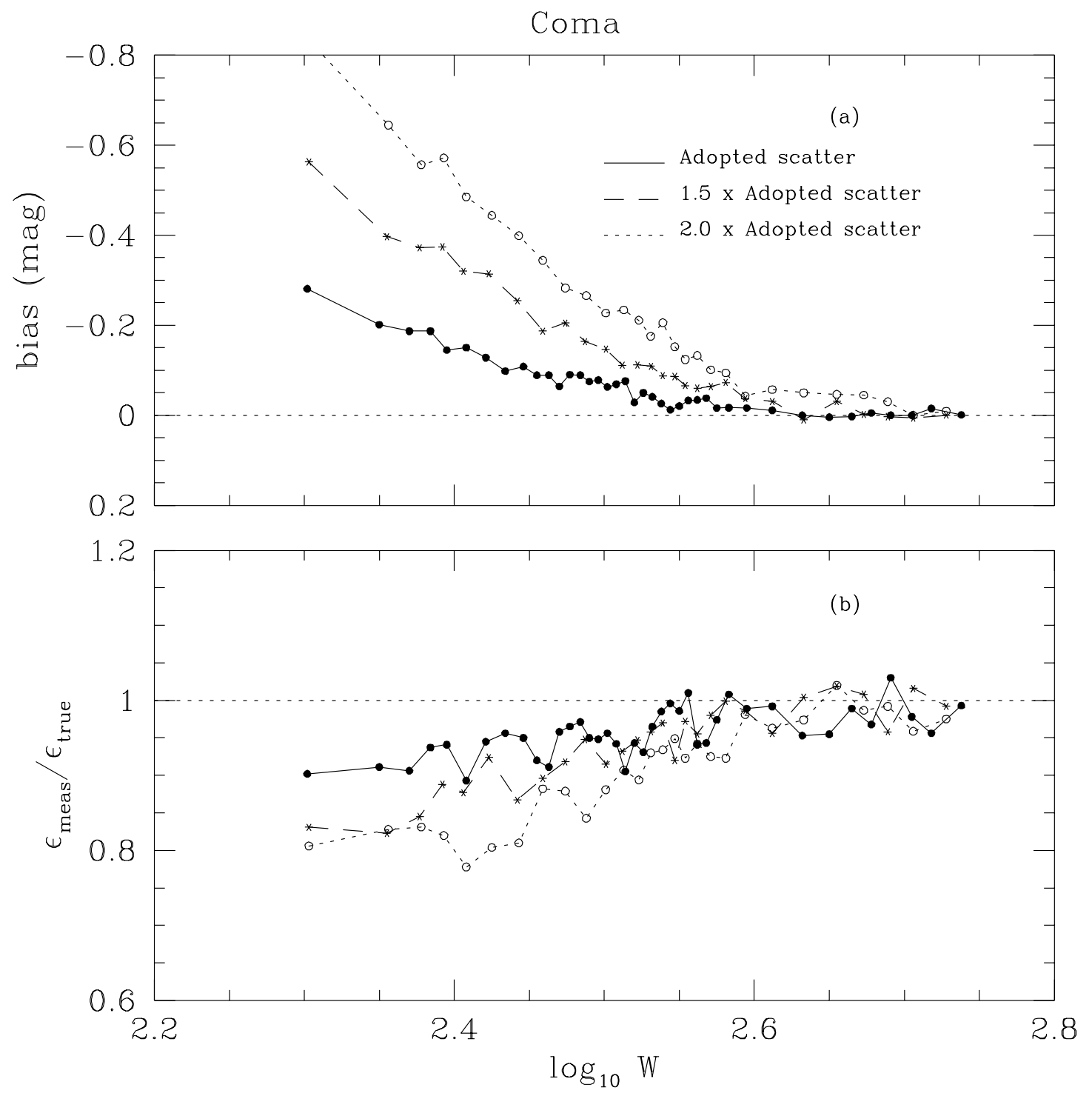

fig. 7 


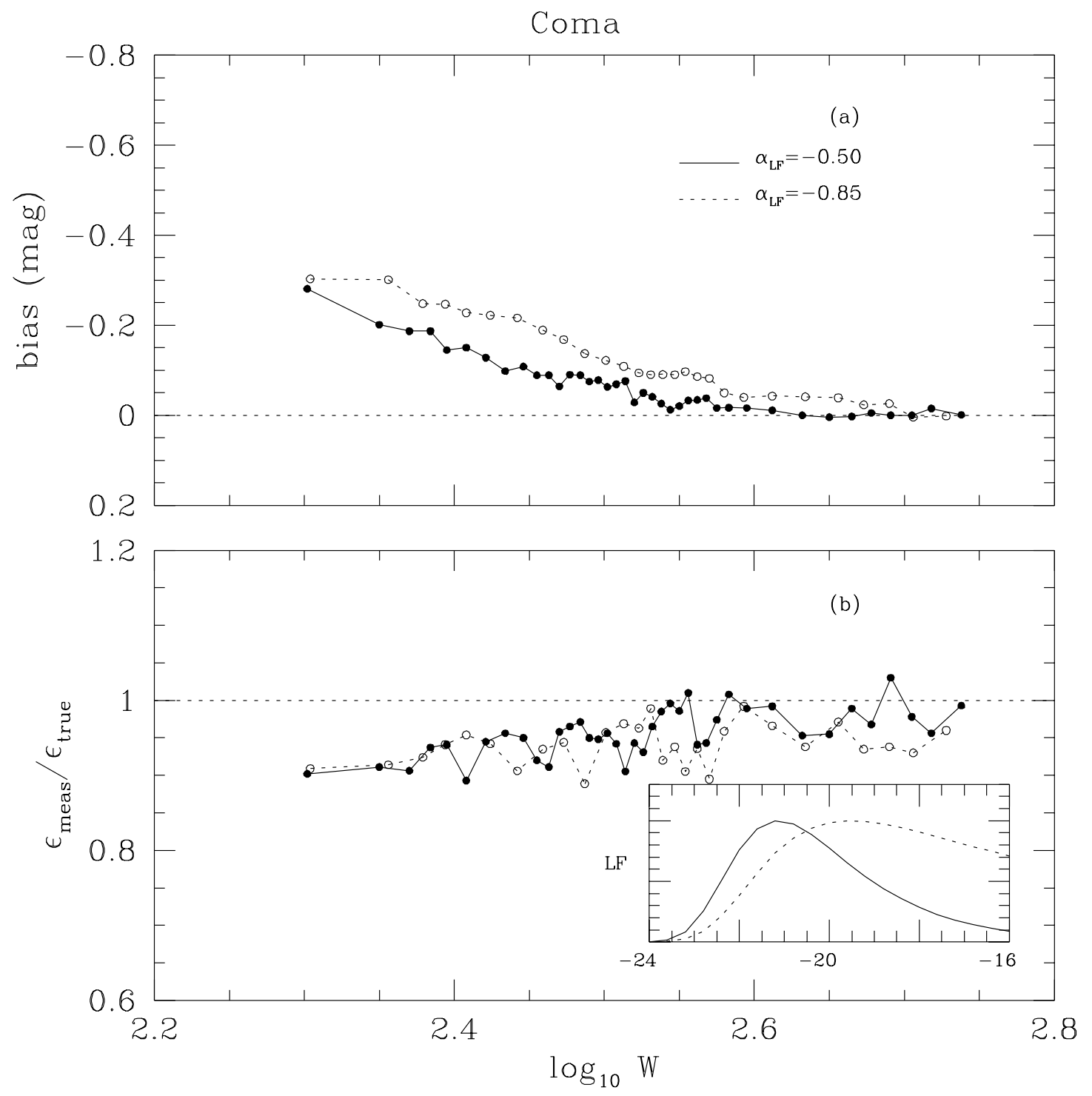

fig. 8 


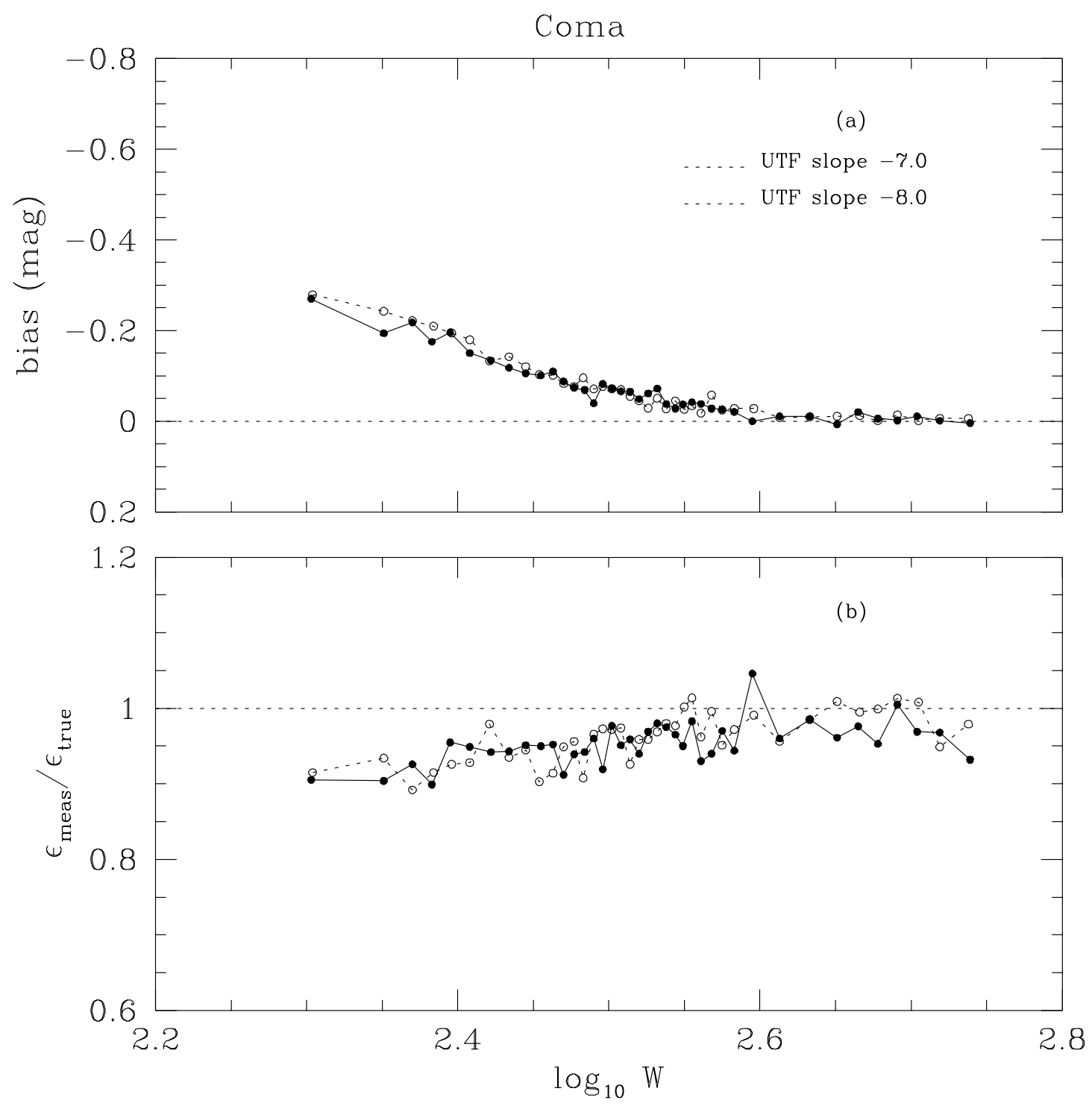

fig. 9 


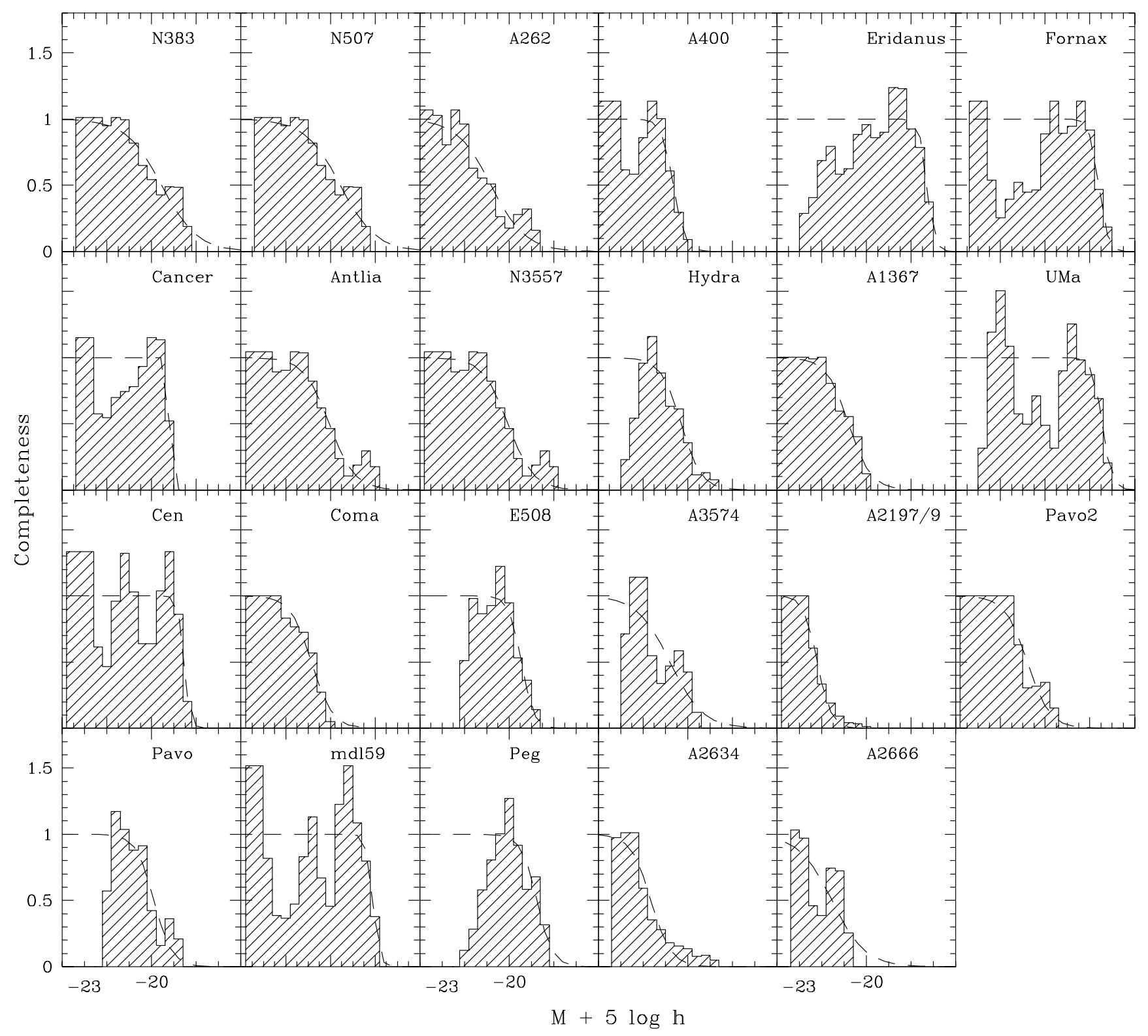

Fig. 10 


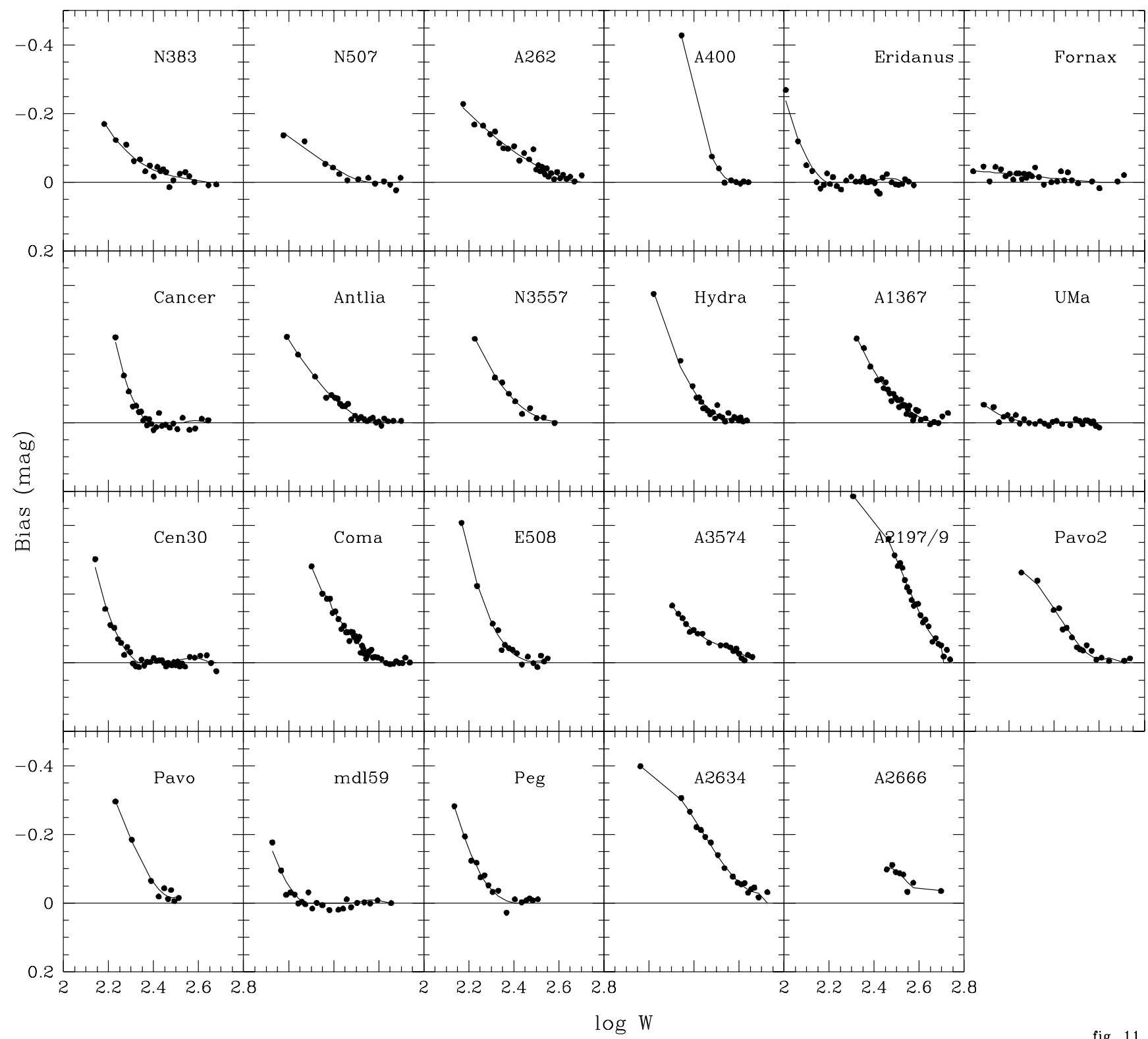

fig. 11 


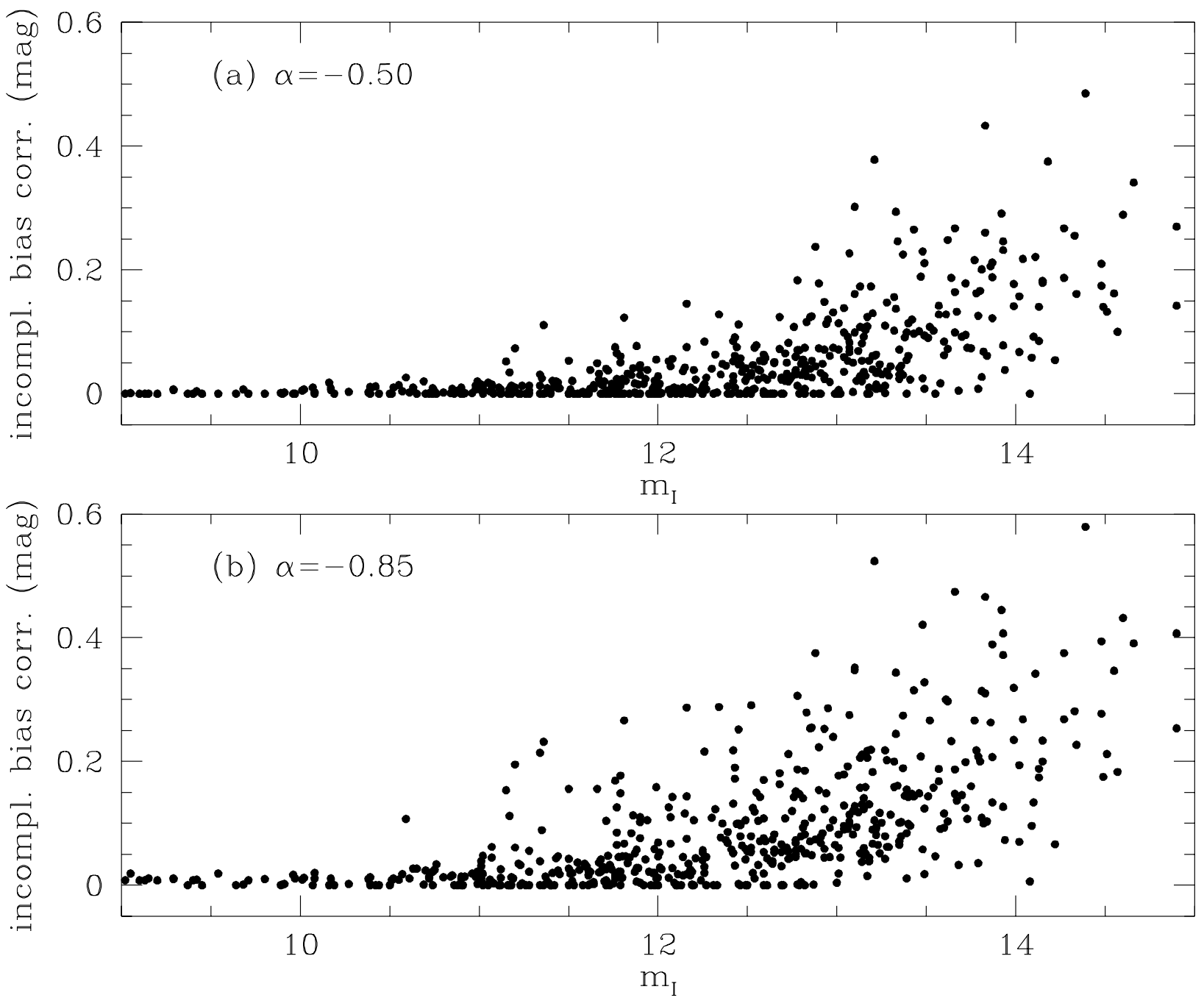




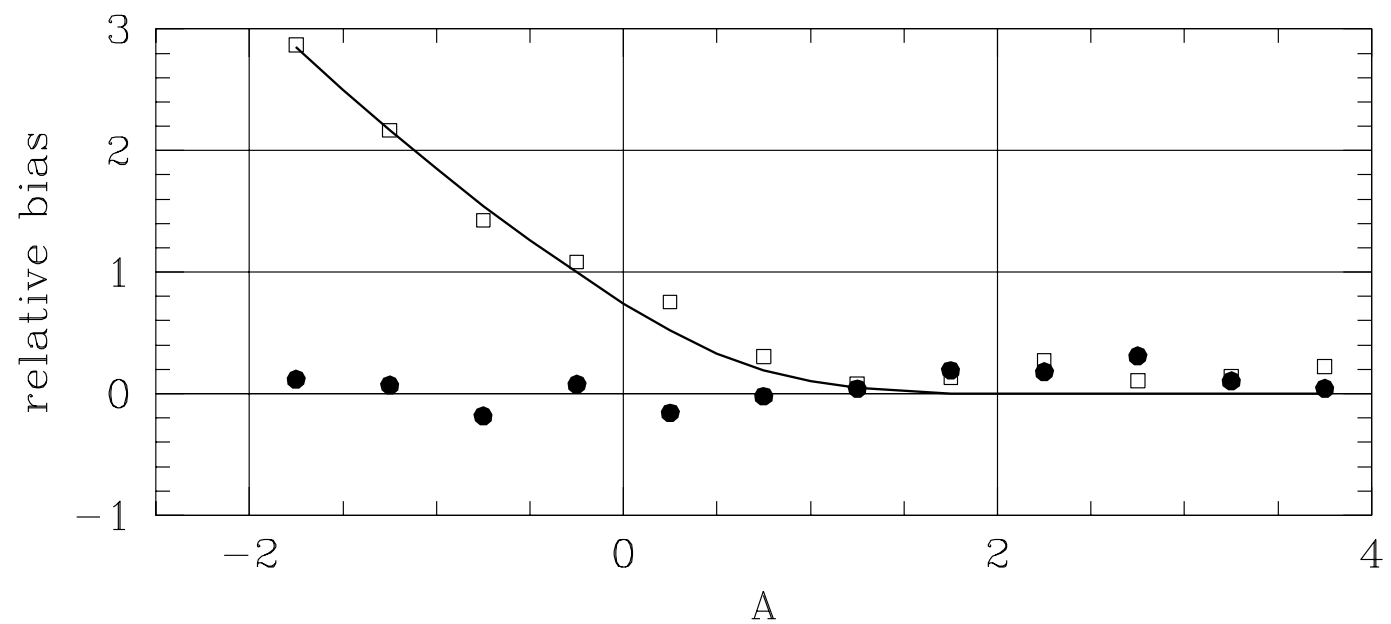

Fig. 13 


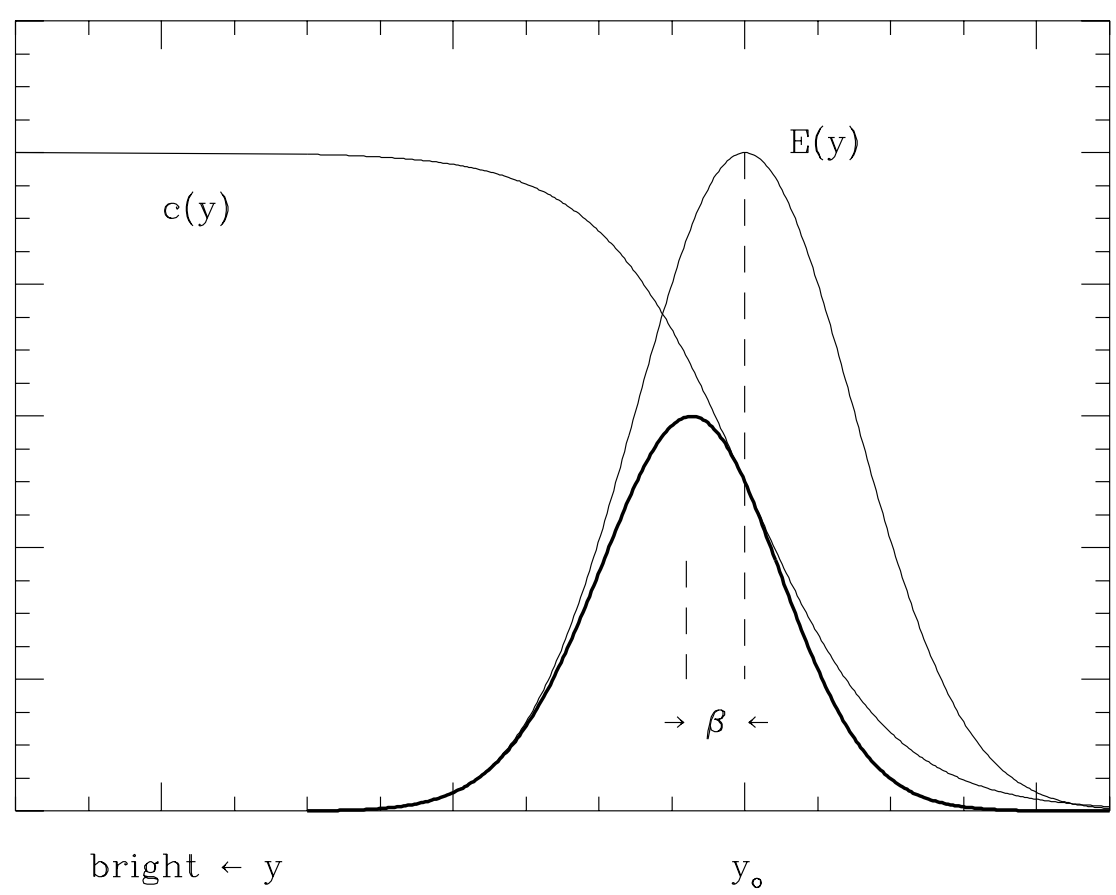

Fig. 14 


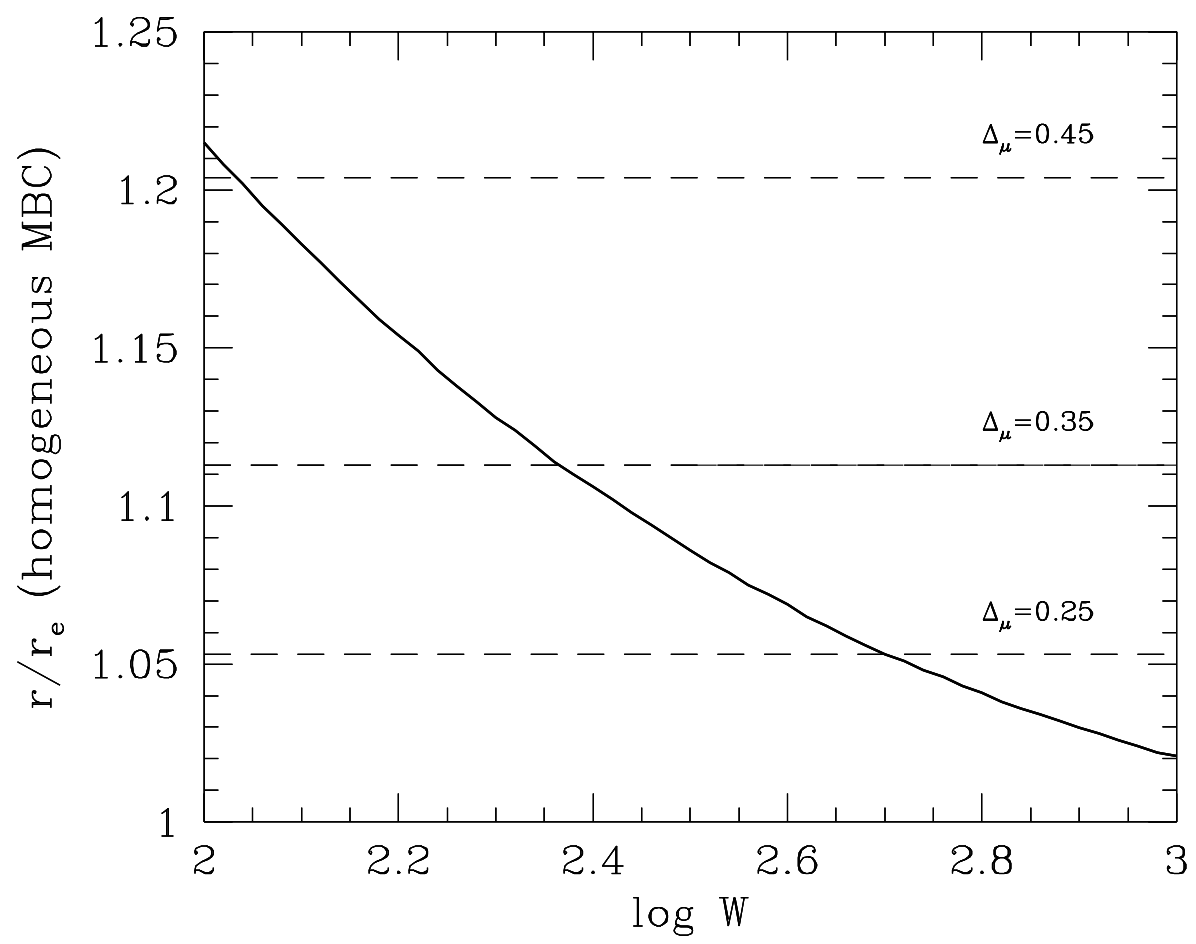

fig. 15 

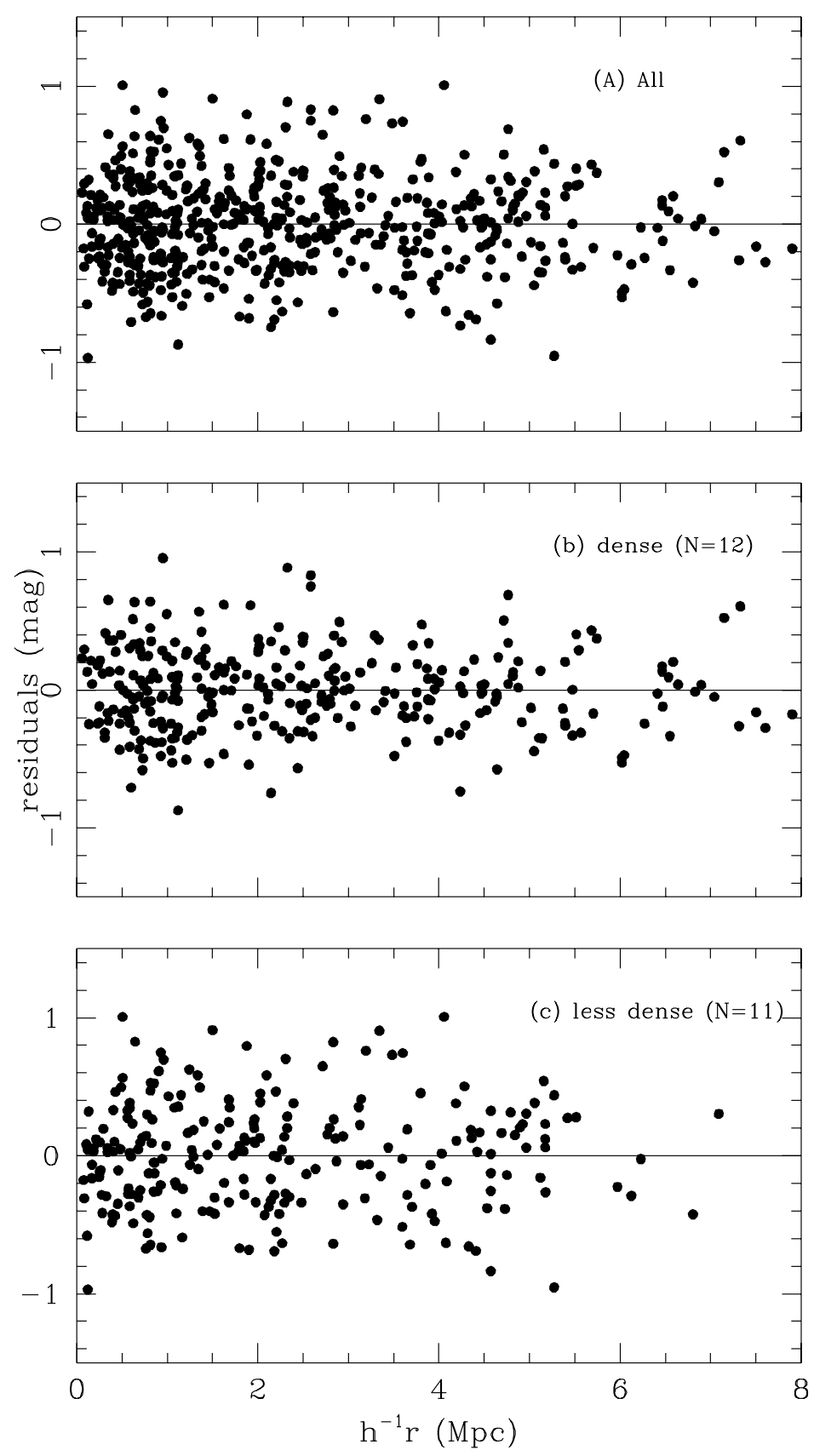

Fig. 16 


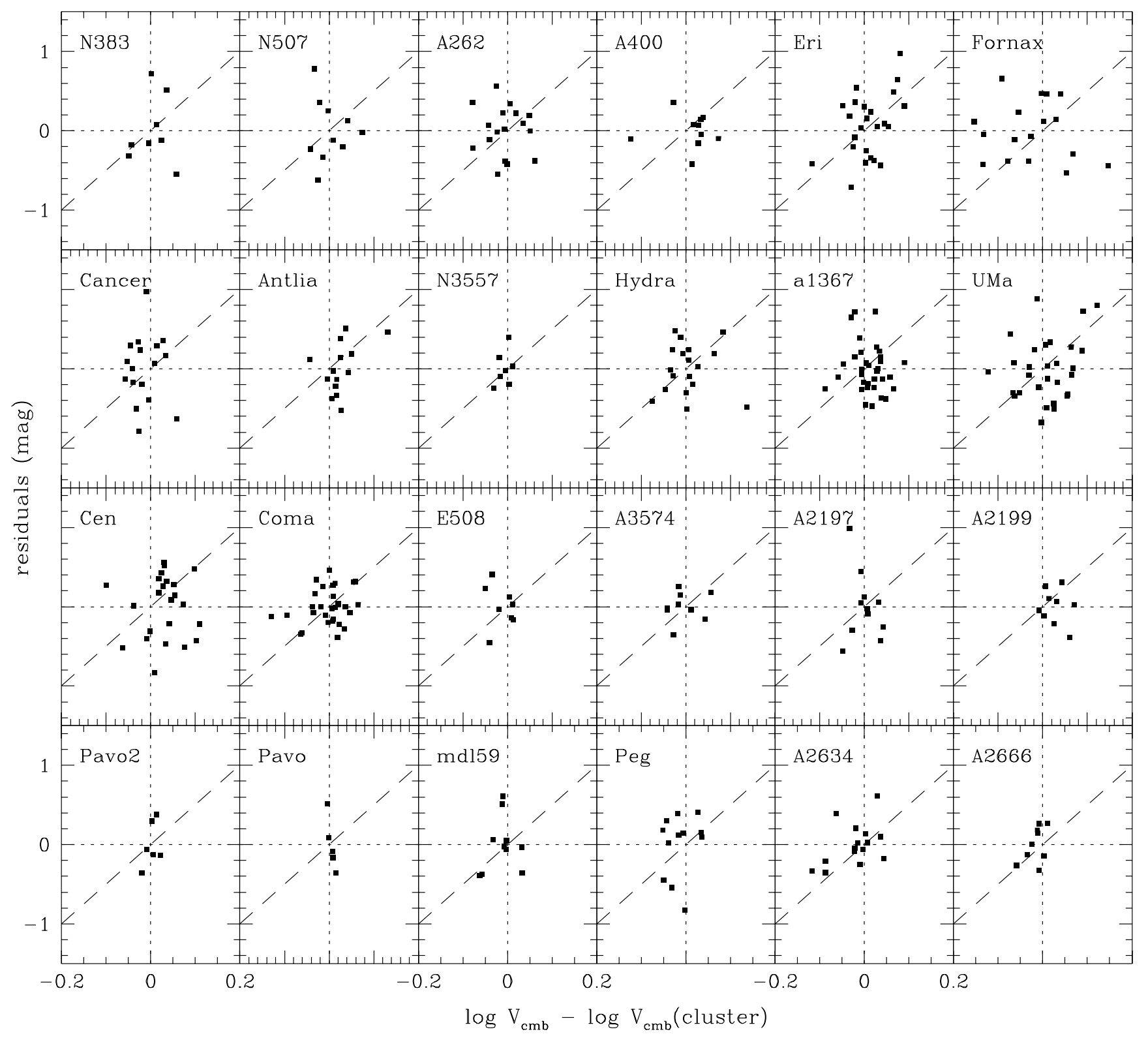




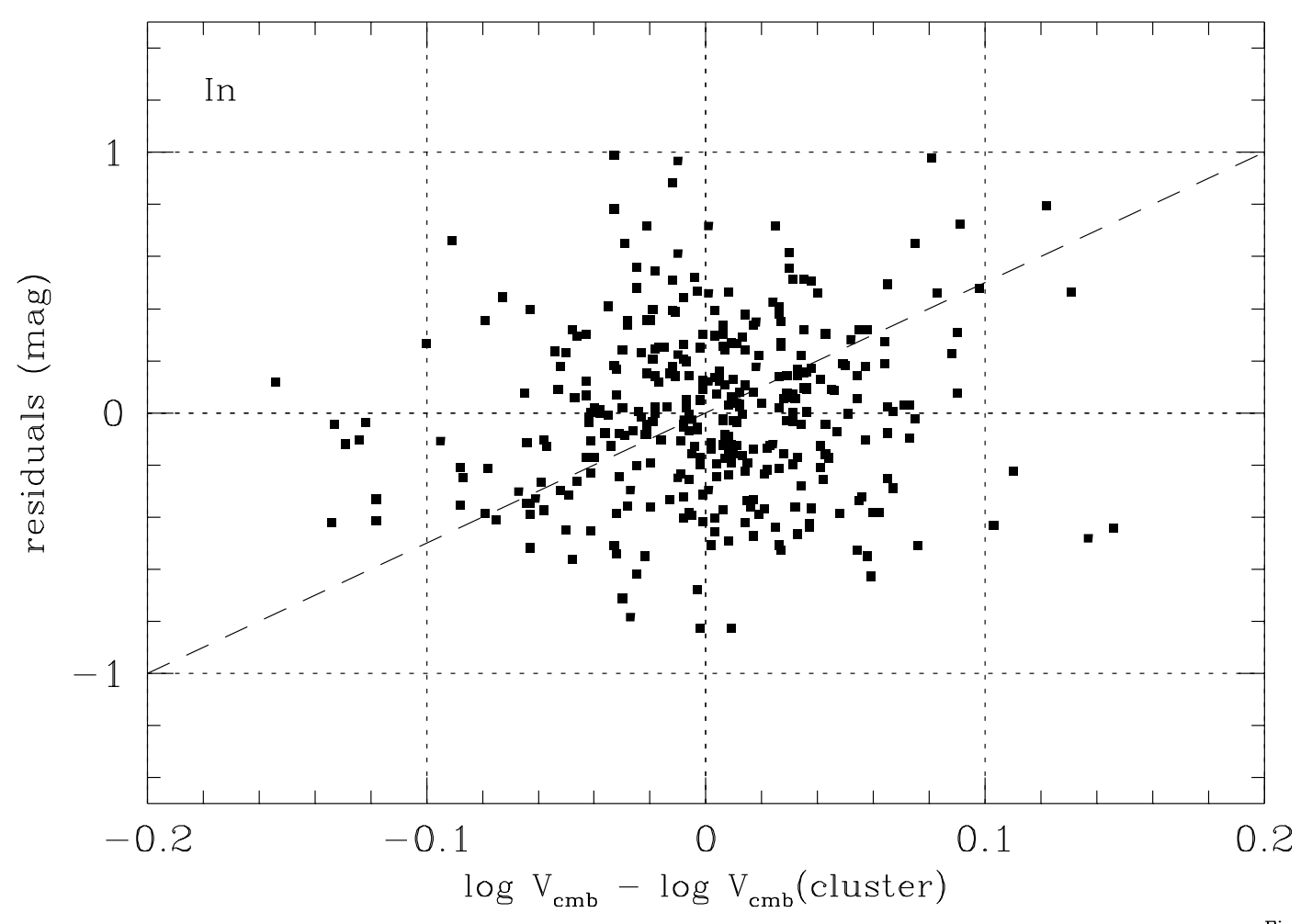

Fig. 20 

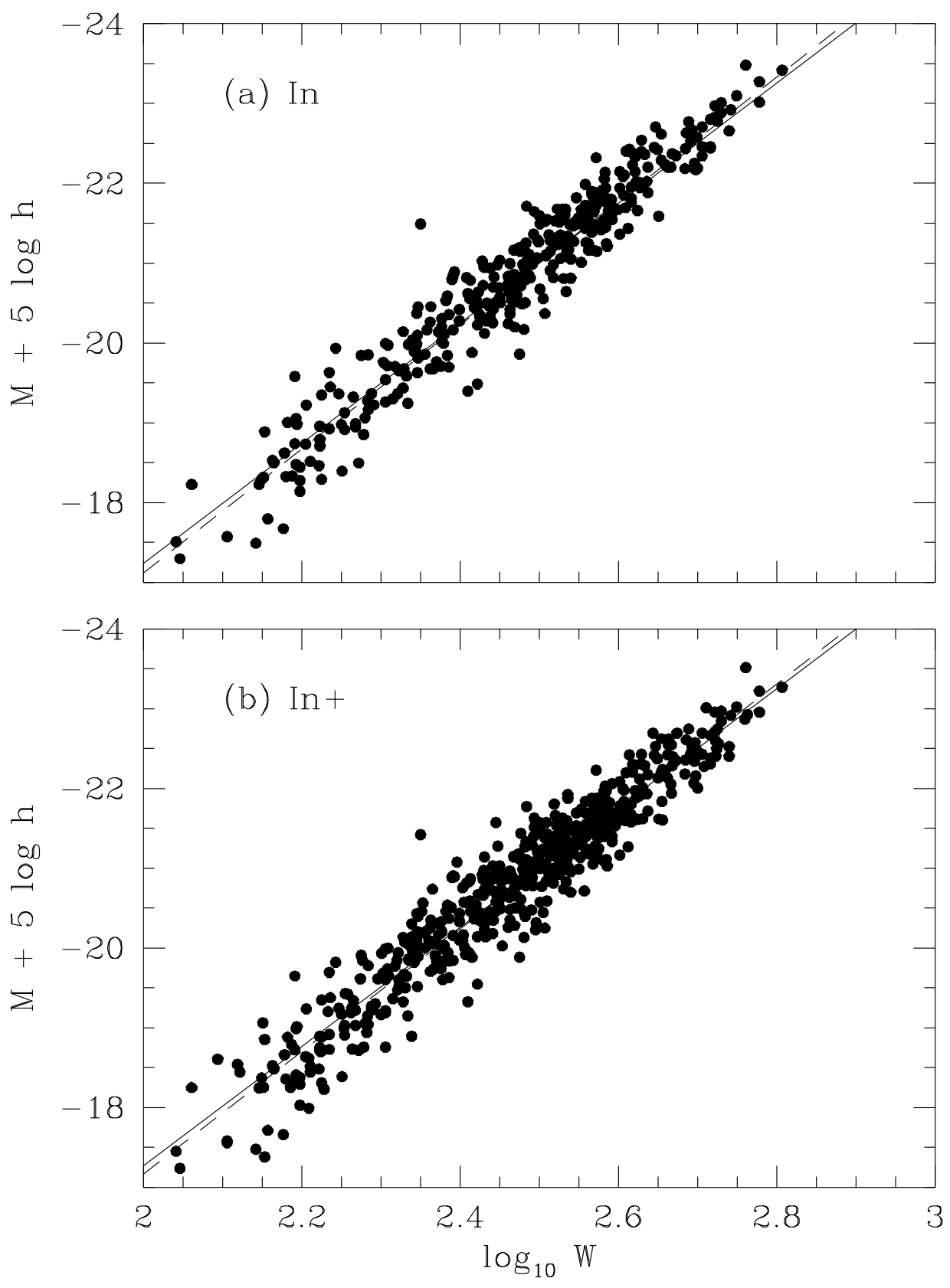

fig. 21 


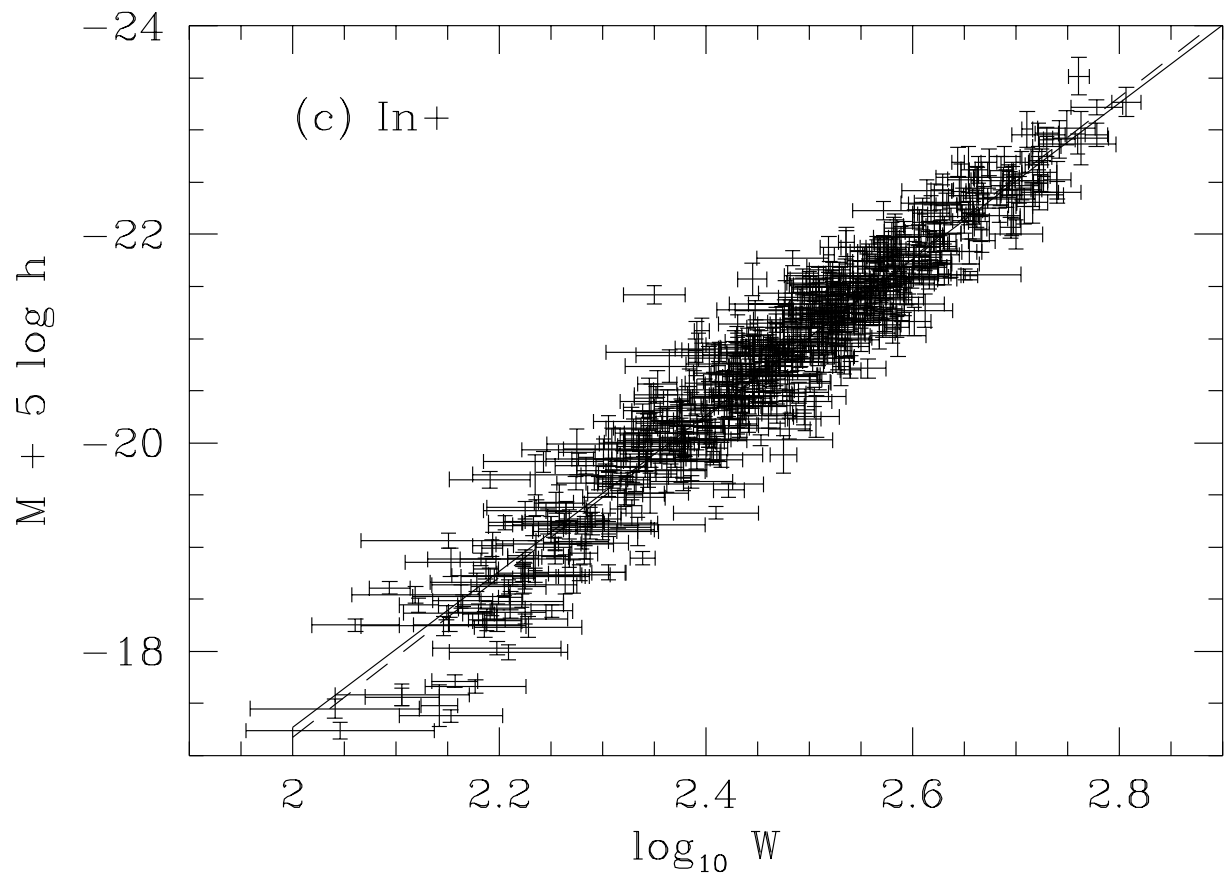

fig. 21(c) 


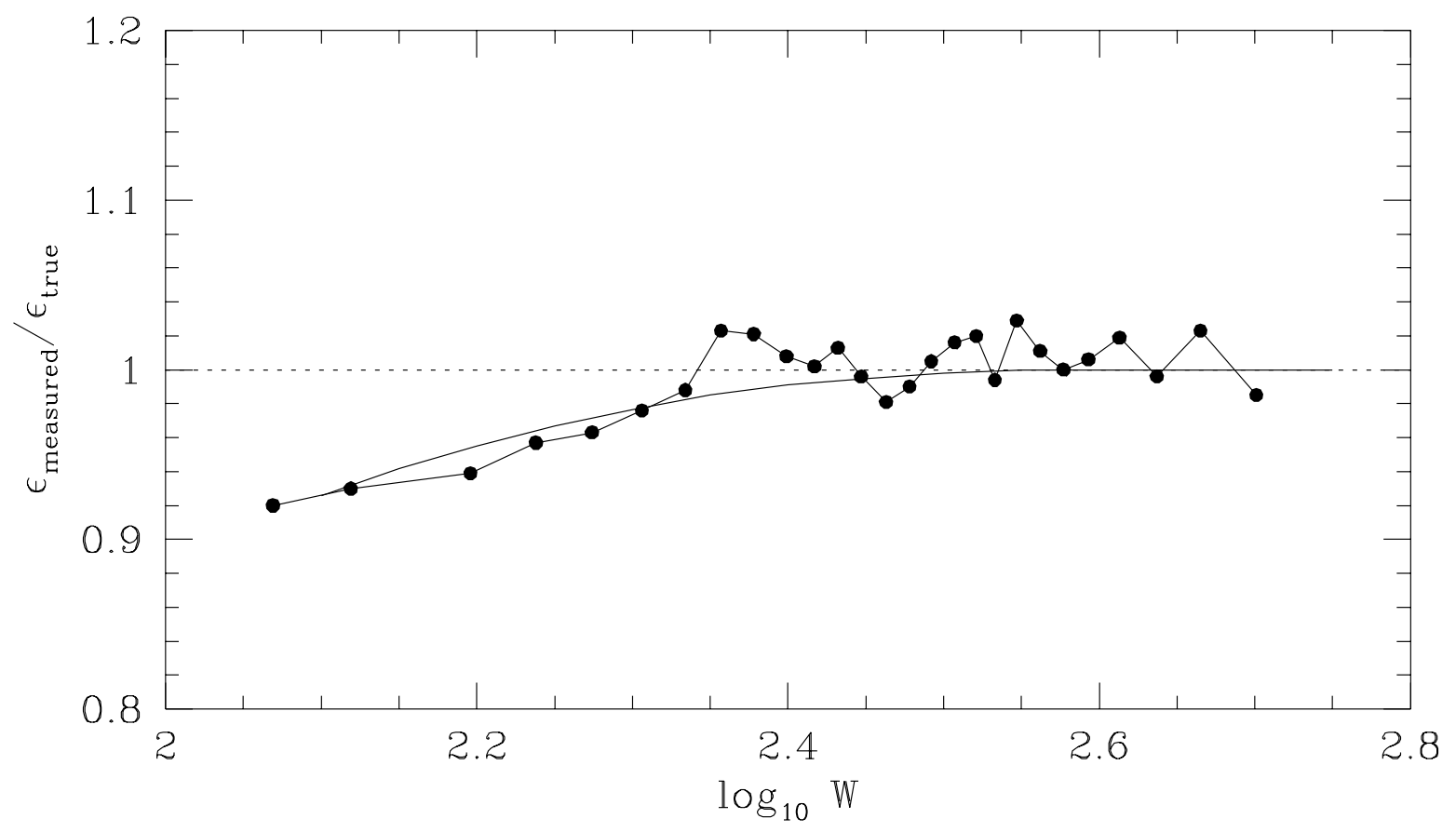

fig. 22 

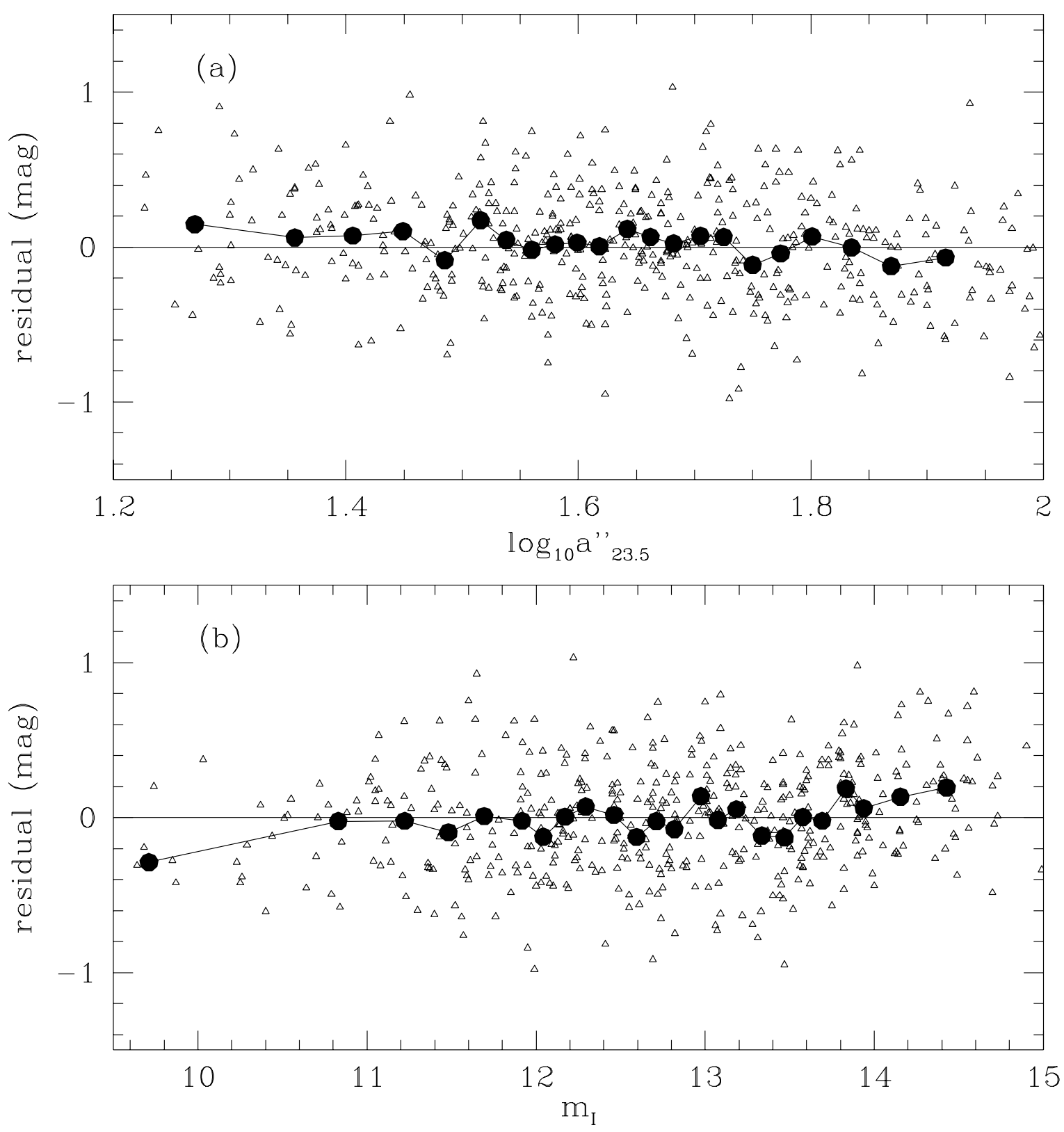

fig. 23 

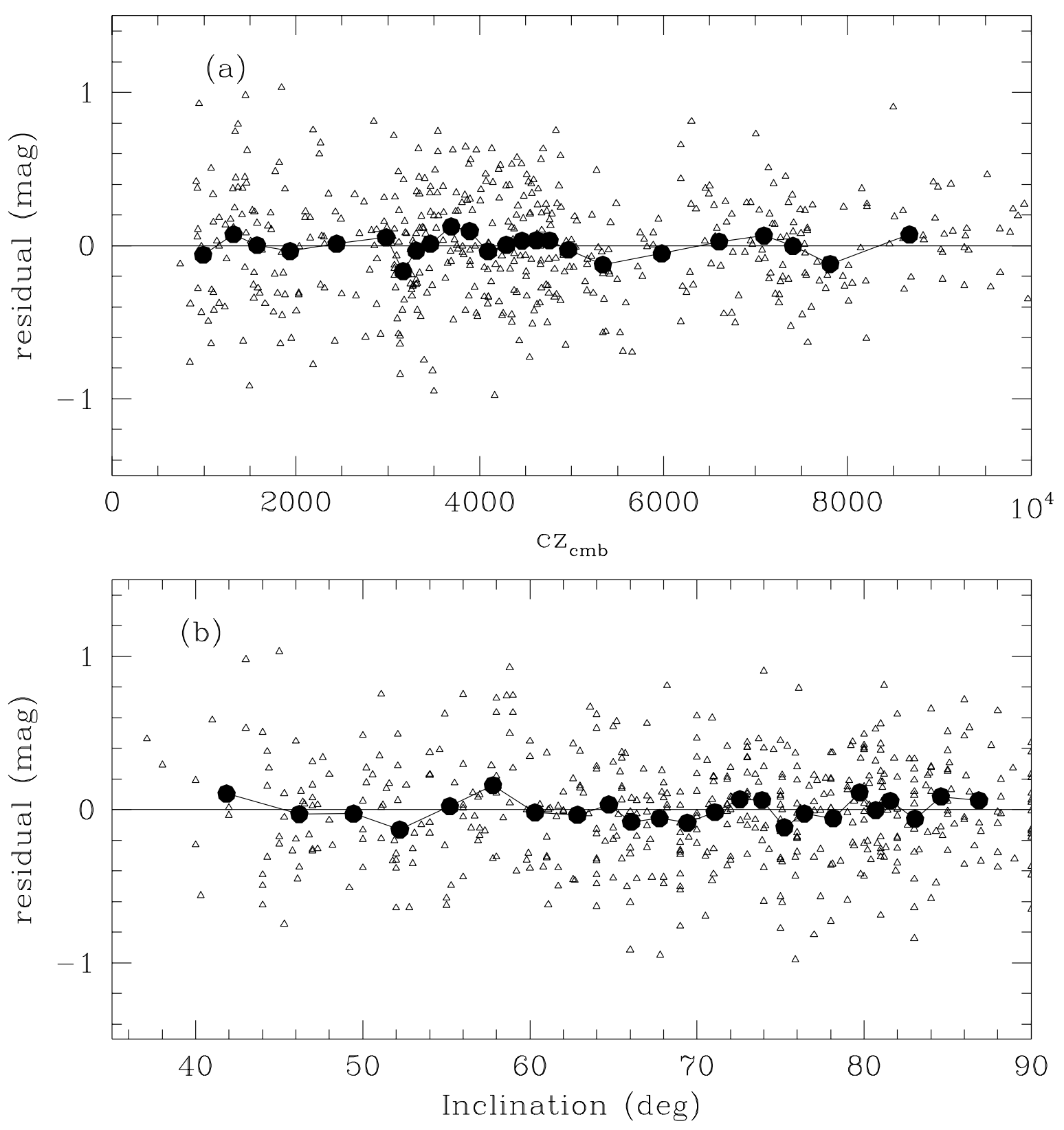

fig. 24 